\title{
A component-based approach to verification and validation of formal software models
}

\author{
Dejan Desovski \\ West Virginia University
}

Follow this and additional works at: https://researchrepository.wvu.edu/etd

\section{Recommended Citation}

Desovski, Dejan, "A component-based approach to verification and validation of formal software models" (2006). Graduate Theses, Dissertations, and Problem Reports. 2495.

https://researchrepository.wvu.edu/etd/2495

This Dissertation is protected by copyright and/or related rights. It has been brought to you by the The Research Repository @ WVU with permission from the rights-holder(s). You are free to use this Dissertation in any way that is permitted by the copyright and related rights legislation that applies to your use. For other uses you must obtain permission from the rights-holder(s) directly, unless additional rights are indicated by a Creative Commons license in the record and/ or on the work itself. This Dissertation has been accepted for inclusion in WVU Graduate Theses, Dissertations, and Problem Reports collection by an authorized administrator of The Research Repository @ WVU.

For more information, please contact researchrepository@mail.wvu.edu. 


\title{
A Component-based Approach to Verification and Validation of Formal Software Models
}

\author{
Dejan Desovski \\ Dissertation submitted to the \\ College of Engineering and Mineral Resources \\ at West Virginia University \\ in partial fulfillment of the requirements for the degree of \\ Doctor of Philosophy \\ in \\ Computer and Information Science \\ Bojan Cukic, Chair \\ Constance Heitmeyer \\ Hany Ammar \\ Katerina Goseva-Popstojanova \\ K. Subramani \\ Edgar Fuller
}

Lane Department of Computer Science and Electrical Engineering

Morgantown, West Virginia

2006

Keywords: D.2 - Software Engineering, D.2.1 - Requirements/Specifications, D.2.4 - Software / Program Verification, D.2.5 - Testing and Debugging, F.3.1 - Specifying and Verifying and Reasoning about Programs

(C) Dejan Desovski, 2006 


\title{
A Component-based approach to Verification and Validation of Formal Software Models
}

\author{
by Dejan Desovski
}

\section{Abstract}

Formal methods for verification of software systems often face the problem of state explosion and complexity. We present a divide and conquer methodology that leads to component based verification and analysis of formal requirements specifications expressed using Software Cost Reduction (SCR) models. The proposed methodology has the following steps: model partitioning, partition verification (either by model checking or testing) and composition of verification results.

We define a novel decomposition methodology for SCR specifications based on min-cut graph algorithms. The methodology identifies components in given SCR specifications and automates related abstraction methods. It also provides guidance on how to perform verification and validation of the formal system models. Further, we present a strategy for verification of modular and decomposable software models. Efficient verification of SCR models is achieved with the use of invariants and proof compositions.

SCR specifications can be executed by the simulator and tested, either automatically (e.g. random testing) or manually, guided by a domain expert using visual interface mimicking the actual system. Some of the identified specification components might be simple enough to allow thorough testing, despite having large state spaces that cause problems with model checking approaches. We define model test coverage measures and develop tools to track the achieved coverage during manual and random testing. Testing and coverage measures provide degree of assurance in the component correctness. Our experimental results also provide insight into the efficacy of random testing approaches for verification of software models.

Experimental validation of our methodology brought to light several concepts that have been advocated in the software development community for a long time: modularity, encapsulation, information hiding and the avoidance of global variables. The advantages of the compositional verification strategy are demonstrated in the case study, which analyses the Personnel Access Control System. Our approach offers significant savings in terms of time and memory requirements needed to perform formal system verification. 
Dedicated to my wife Eli,

and my family,

for their unlimited support and understanding. 


\section{Acknowledgments}

Many people need to take credit for making this work possible. First of all, I would like to thank my advisor Dr. Bojan Cukic for his more than generous support during the years I worked on this dissertation. His unlimited wisdom, cheerful comments, academic excellence, have inspired me during the difficult days and showed me the right way in pursuing my interests. His guidance and education given to me is priceless.

I would like to thank Constance Heitmeyer for the wonderful internships I have spent at the Naval Research Laboratory. The time and discussions with her on SCR, Requirements and Software Engineering, and every other topic were invaluable. At NRL I had chance to meet and enjoy the company of Ramesh Bharadwaj, Myla Archer, Elizabeth Leonard, Ralph Jeffords, James Kirby, Michael Colon, Angelo Gargantini, and have fun time with the other interns visiting the Lab.

The PhD committee members: Dr. Katerina Goseva-Popstojanova, Dr. Hany Ammar, Dr. Eddie Fuller, and Dr. K. Subramani, provided excellent support and insightful comments and discussions during the work on the dissertation. I also learned a lot taking their classes at WVU and collaborating on different projects.

My student colleagues helped me to stay sane during the whole ordeal and created excellent work atmosphere: Nevena Samoska, Petar Popic, Martin Mladenovski, Yan Liu, Vijai Gandikota, Nick Bartlow, Sampath Yerramalla, Yue Jiang, Paola Bracchi, David Owen. I am also very grateful to Nevena Stojanovic for the time she spent correcting and improving the dissertation writing.

This work was sponsored by National Science Foundation grant no. CCF-0093315 entitled "CAREER: Software Reliability Assessment for High Assurance Systems”, and NASA Contract no. NCC5-685, task 3A, entitled "A Compositional Approach to Validation of Formal Models". 


\section{Table of Contents}

ABSTRACT .............................................................................................................................................................................. ii

TABLE OF CONTENTS .......................................................................................................................

LIST OF TABLES................................................................................................................................vii

LIST OF FIGURES............................................................................................................................ viii

CHAPTER 1. INTRODUCTION……………........................................................................................ 1

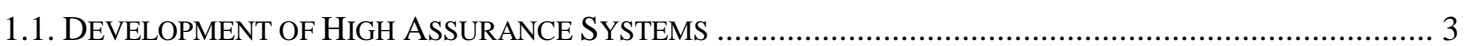

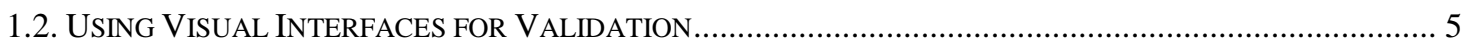

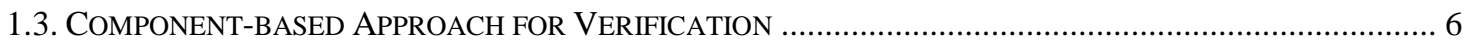

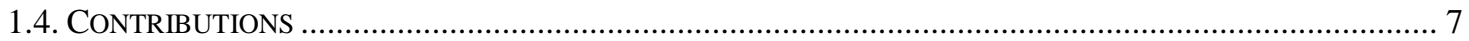

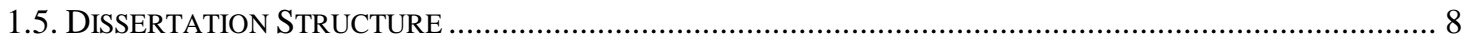

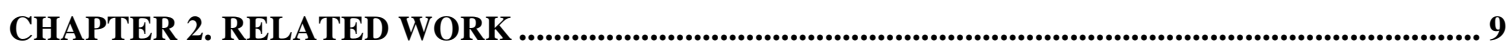

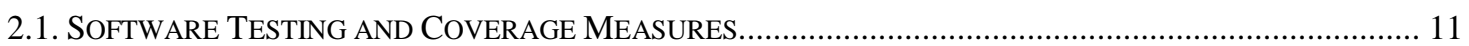

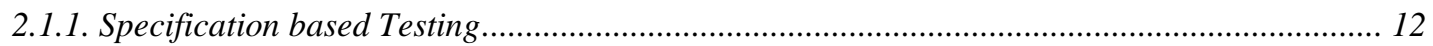

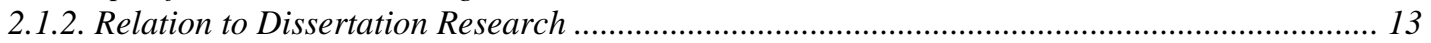

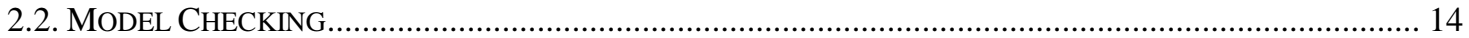

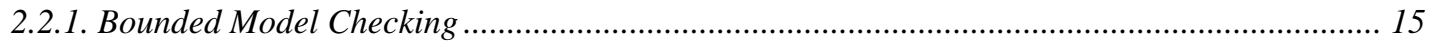

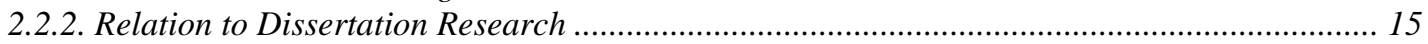

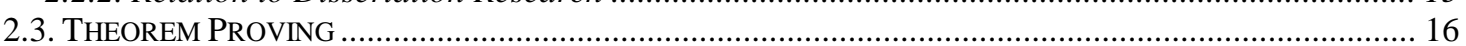

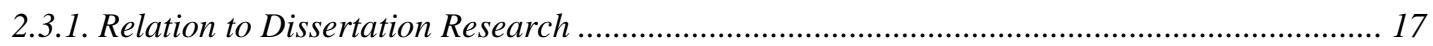

CHAPTER 3. BACKGROUND AND A MOTIVATING EXAMPLE .............................................. 18

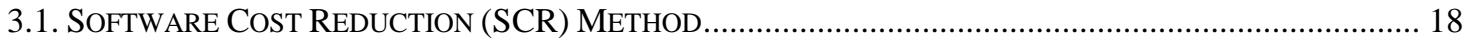

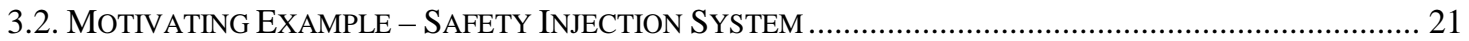

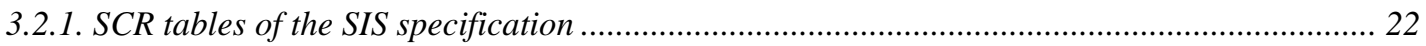

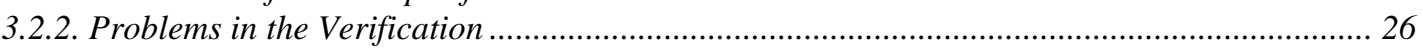

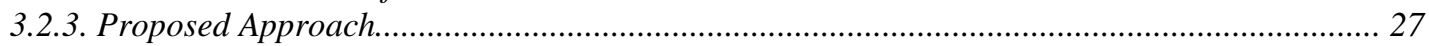

3.3. THEORETICAL BASIS FOR THE VERIFICATION OF DECOMPOSABLE MODELS ....................................... 28

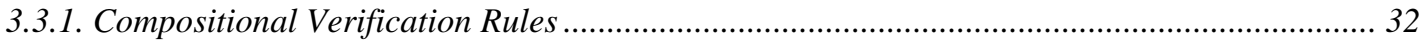

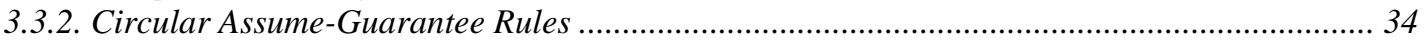

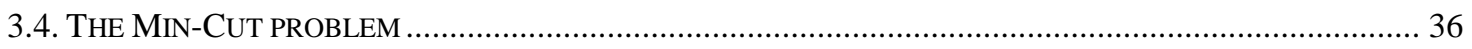

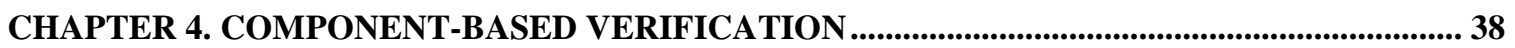

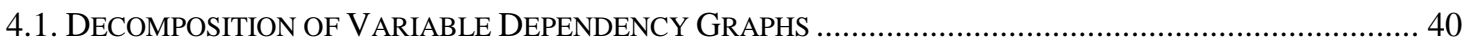

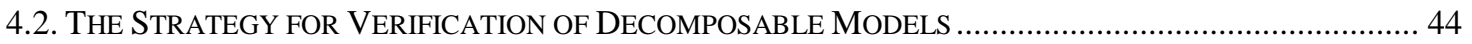

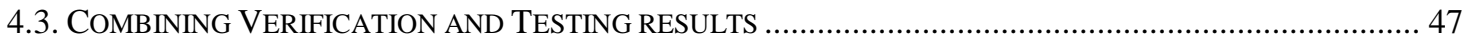

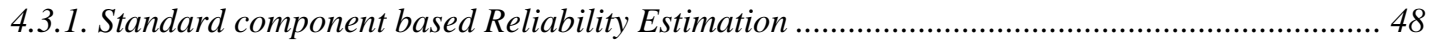

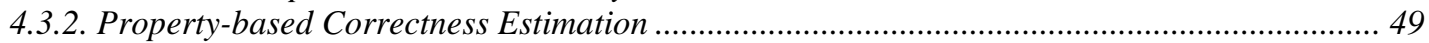

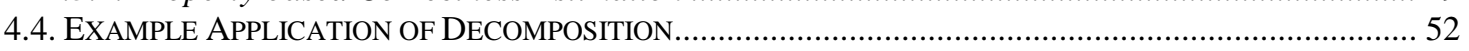

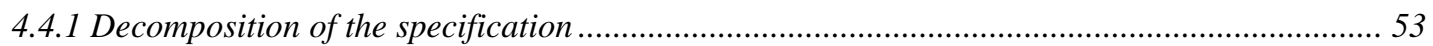

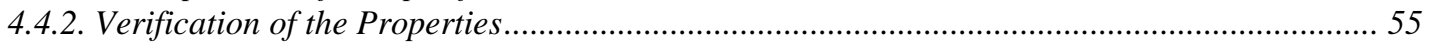

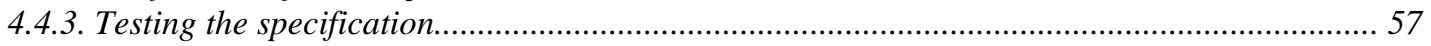

CHAPTER 5. TESTING AND COVERAGE OF FORMAL MODELS ..........................................59

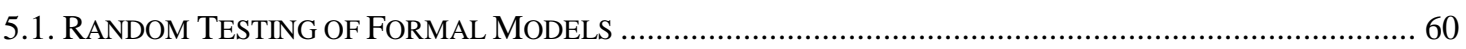

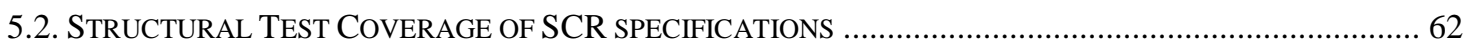

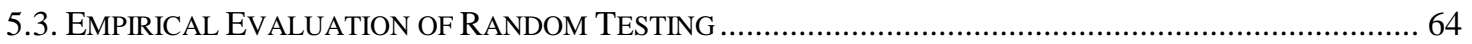

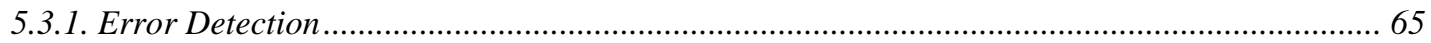

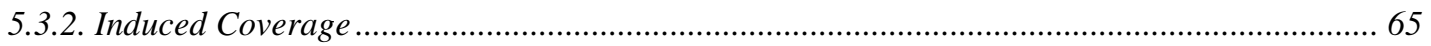


CHAPTER 6. USING VISUAL INTERFACES FOR VALIDATION .................................................68

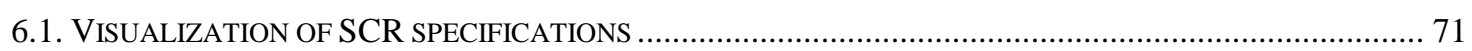

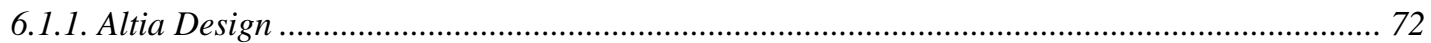

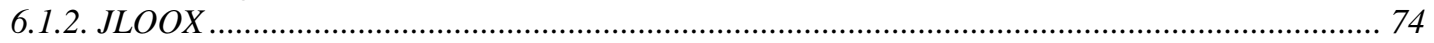

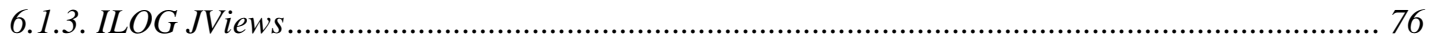

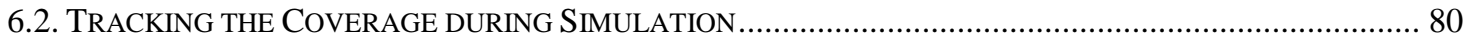

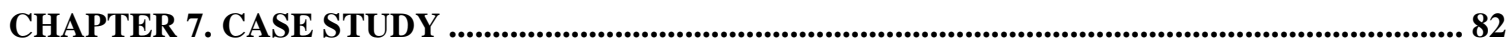

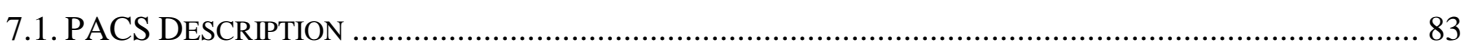

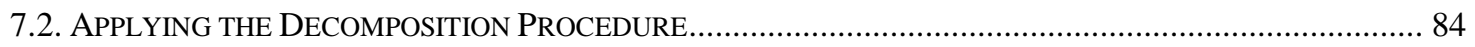

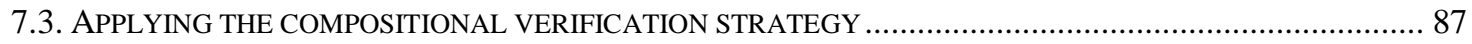

CHAPTER 8. CONCLUSIONS AND FUTURE WORK ........................................................................... 91

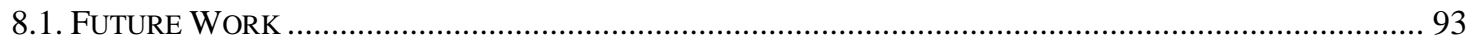

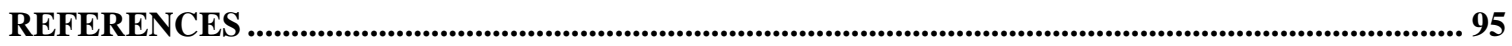

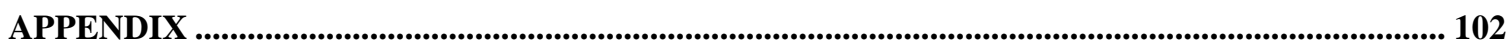

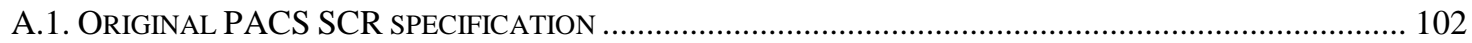

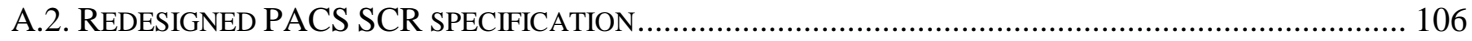




\section{List of Tables}

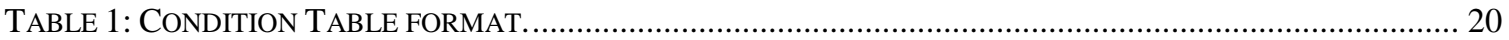

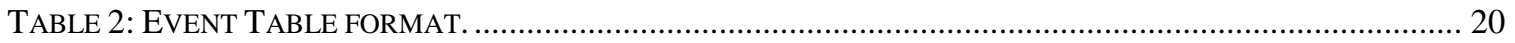

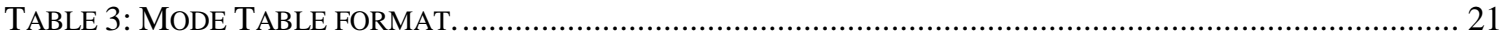

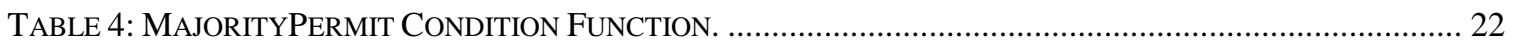

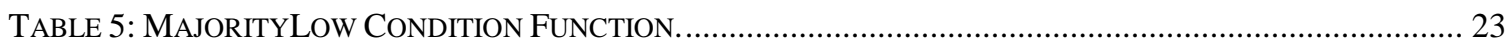

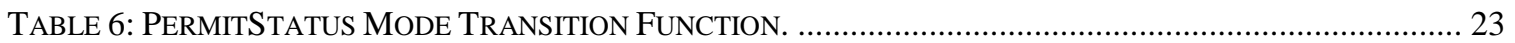

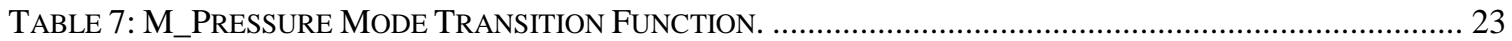

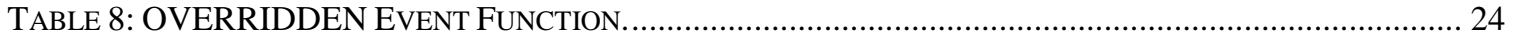

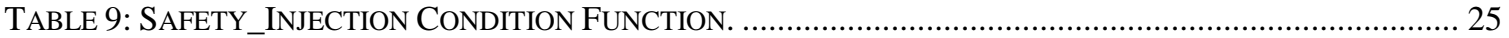

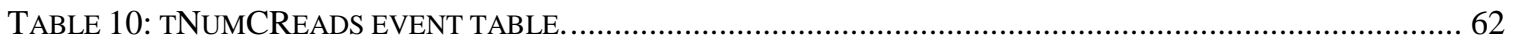

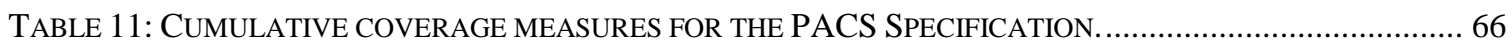

TABLE 12: THE RESOURCES NEEDED AND NUMBER OF VERIFIED PROPERTIES FOR EACH COMPONENT............ 90 


\section{List of Figures}

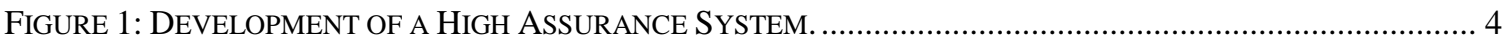

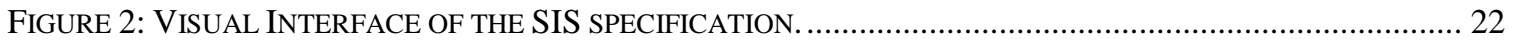

FIGURE 3: DEPENDENCY GRAPH OF THE SAFETY INJECTION SCR SPECIFICATION....................................... 25

FIGURE 4: EXECUTION OF THE SPIN ON THE COMPLETE MODEL. ……....................................................... 26

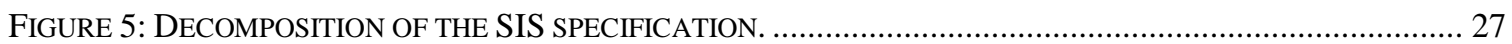

FIGURE 6: TRANSFORMATION OF THE DEPENDENCY GRAPH TO UNDIRECTED GRAPH. ............................... 42

FIGURE 7: MINIMUM CUT OF THE TRANSFORMED GRAPH OF A VARIABLE DIRECTLY DEPENDANT ON SEVERAL

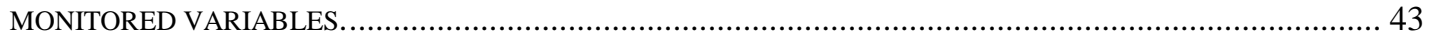

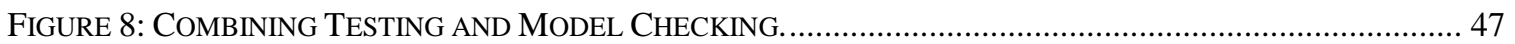

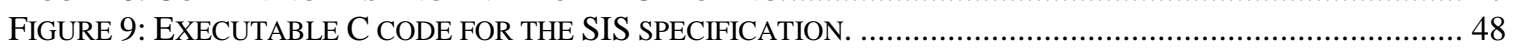

FIGURE 10: DEPENDENCY GRAPH OF THE SAFETY INJECTION SCR SPECIFICATION. ..................................... 52

FIGURE 11: DEPENDENCY OF THE VARIABLES ON THE "OLD" VALUES...................................................... 53

FIGURE 12: DECOMPOSITION OF THE COMPLETE DEPENDENCY GRAPH OF THE SIS SPECIFICATION. ............... 54

FIGURE 13: DECOMPOSITION OF THE "NEW" DEPENDENCY GRAPH OF THE SIS SPECIFICATION....................... 54

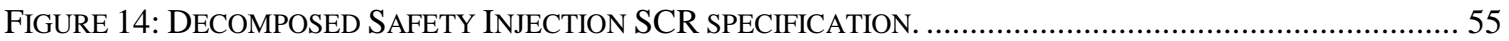

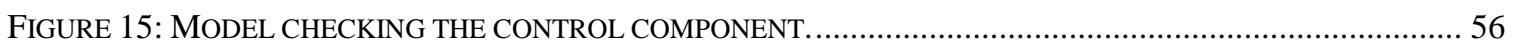

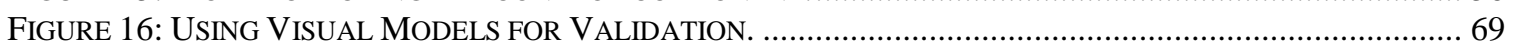

FIGURE 17: SCR SIMULATOR WITH THE DEFAULT INTERFACE FOR THE SAFETY INJECTION SYSTEM.............. 71

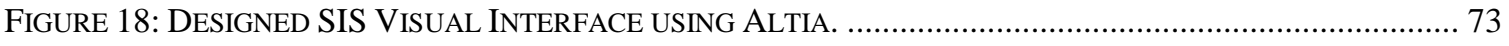

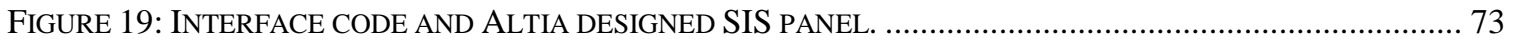

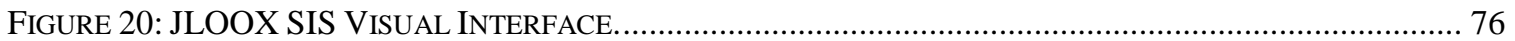

FIGURE 21: ILOG JVIEWS SIS INTERFACE WITH THE VALUESOURCE CONNECTIONS................................... 78

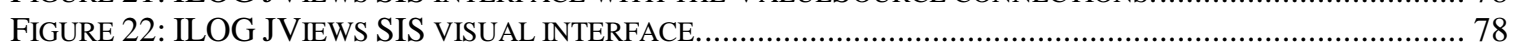

FIGURE 23: SCR SIMULATOR PALETTE WITH THE ILOG JVIEWS METER OBJECT........................................... 79

FIGURE 24: PROTOTYPE TOOL FOR TRACKING THE COVERAGE OF THE EXECUTED SIMULATION. ..................... 81

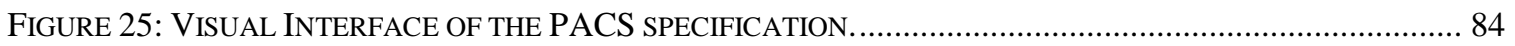

FIGURE 26: DECOMPOSITION OF THE REFACTORED PACS SPECIFICATION. ................................................ 86

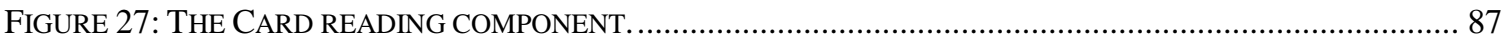

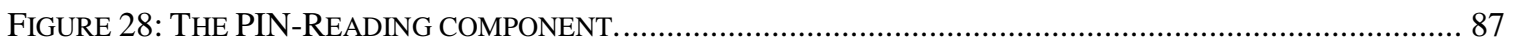

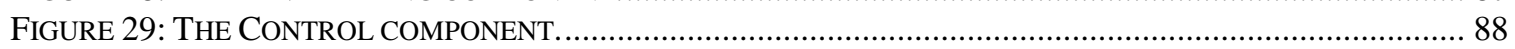




\section{Chapter 1}

\section{Introduction}

By definition, a high assurance system is a system for which compelling evidence is required to demonstrate that it delivers its services in a manner that satisfies certain critical properties. Consequently, during the development of a high assurance system we must prove that it does not contain any faults or, if that is out of our reach, that failures are highly unlikely to occur.

High assurance software systems continue to pose significant challenges for verification and validation. Software industry relies mostly on informal methods (e.g., code review or testing) for quality assurance purposes. However, even the most experienced quality assurance engineers can often overlook faults.

Formal methods hold a promise for the development of provably correct software applications. Despite enthusiasm in the research community, formal methods are rarely used in software industry. Continual complaints regarding the difficulty of applying them to practical software applications are due, in part, to scalability problems. The challenges of formal approaches are caused by the large state-spaces and the complexity of any practical software application. The automated formal methodologies, such as model checking, often hit the state-space explosion barrier, while the approaches involving 
theorem proving require advanced skills and knowledge. Several techniques have been researched to combat the complexity and state-space explosion. For example, partial order reduction [58], abstractions [12][23], assume guarantee reasoning [69] and other approaches have been proposed, but practical applications of formal methods to real-life software systems remain as uncommon as they were a decade ago.

In order to apply formal methods, the underlying software model must be recorded in some form of a formal notation. For our research purposes, we selected the mature Software Cost Reduction (SCR) requirements specification methodology [9]. Typical SCR requirement specification consists of two parts. The operational part describes system operation, while the property-based part encodes the logical and temporal properties that the system must satisfy in operation. SCR tables in the operational part represent the system as a finite state machine, while the properties are first order logic formulas representing state or transition (two-state) invariants which must hold for the system. Having this separation makes it possible to perform verification and detect possible inconsistencies using formal methods like theorem proving or model checking. When we talk about "verification" in the remainder of the dissertation, we are referring to the formal consistency verification between the stated properties and the operational description of a given system.

The role of the properties is similar to the notion of checklist used for certification of systems in other engineering disciplines. If all items (properties) on the checklist are satisfied, the system is certified for use; if not the engineer must present sufficient evidence that they are true so that the system can become certified. Consequently, this list must be complete in the sense that it must contain all needed properties that establish the correctness of the system.

Proving the correctness of these formal software models is one of the most challenging tasks in the development of High Assurance Systems. Validation - (showing that we have modeled the right system) is usually done by simulation or testing, if the model is executable, or by manual inspection otherwise, using the human cognitive process to bridge the gap between the formal representation and system itself. Verification, i.e., 
ensuring that the implementation meets the requirements, is usually done by applying a formal method, like model checking or theorem proving. Combing both procedures, the formal analysis and execution-based testing, for the purpose of validation and verification of software models has been an area of active research for almost a decade, but few practical results emerged.

Since software requirements specifications are on a higher level of abstraction than the code, it is easier to perform different types of analyses. However, large practical requirements specifications still face state space explosion and complexity problems. When the formalism is executable, as the operational part of SCR is, it is also feasible to perform simulation and testing as means of validating the specification. Jointly, various verification and validation methods can complement each other in reaching sufficient level of confidence in system correctness.

\subsection{Development of High Assurance Systems}

The development of High Assurance systems (Figure 1) can be divided in three phases. The first one is Formalization Phase, when we map informal problem description (usually given in natural language) into formal framework (some mathematical constructs), and it is completed when a formal specification has been obtained. The second phase is the Implementation Phase, when we use the formal specification to obtain executable code. The third phase is Testing and Analysis, where we would like to prove that the specification correctly captures all user requirements, and that the developed code is correct with respect to the specification. If any errors are discovered, they must be traced back to their origin (specification or implementation) and corrected.

Proving the correctness of the developed formal specification with respect to the requirements is one of the most important and most difficult tasks in this process. Studies have shown that significant number of faults in real systems can be traced back to the requirements specifications [48]. 
Since both the specification and the implementation are formal objects (they have precise and well-defined semantics), it is possible, in principle, to formally verify the correctness of the implementation with respect to the specification by using mathematical proof techniques. Alternatively, we can use formal rewriting and transformation systems in order to "translate" the formal specification in some high level programming language like C or Java [26].

However, we can never be sure that the formalization phase has been done correctly in the first place. This uncertainty arises from the fact that the formalization process maps informal knowledge into a formal framework.

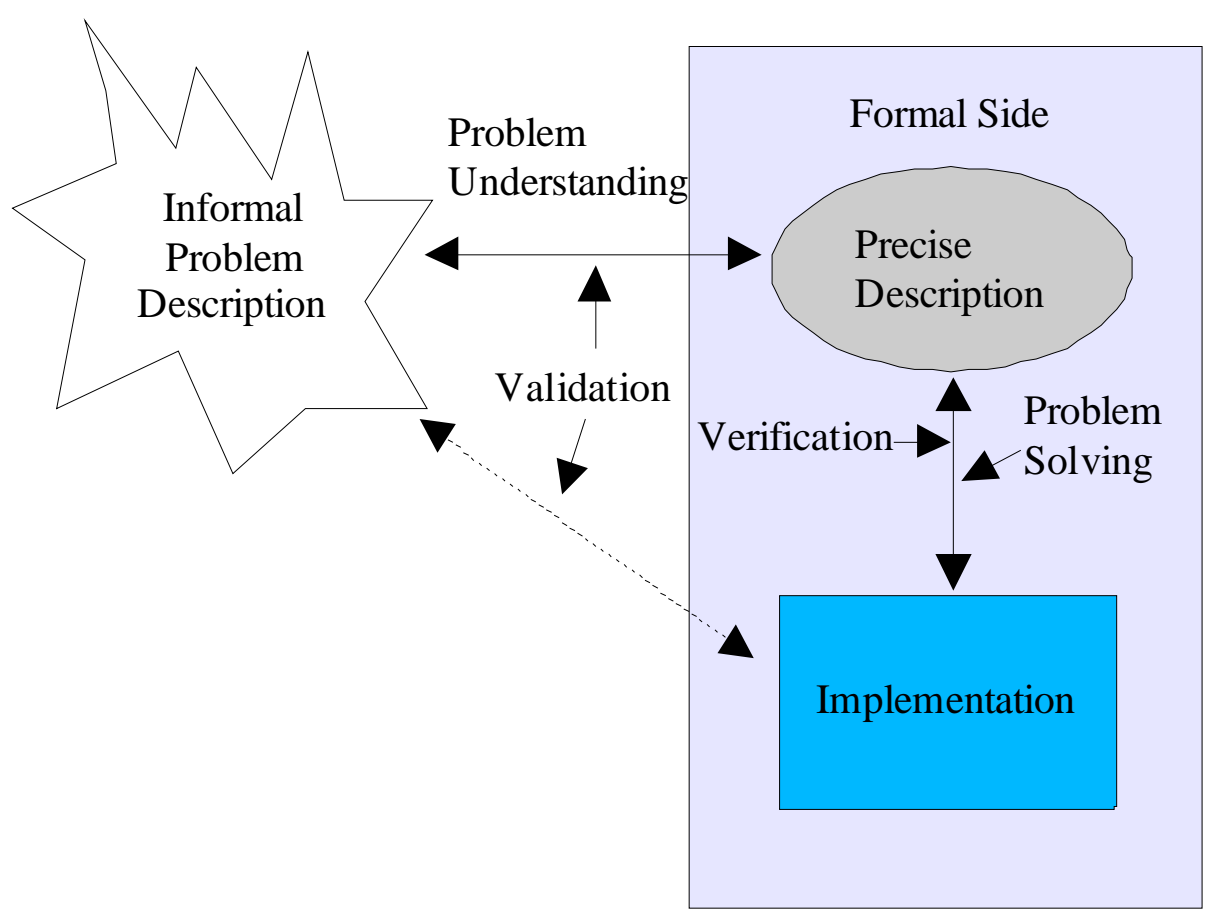

Figure 1: Development of a High Assurance System.

The basis of our approach is the SCR (Software Cost Reduction) formal representation and its finite state model of the system. SCR specifications deal with reactive systems that monitor variables in the environment and react accordingly changing the controlled variables in a single step. There are no explicit loops in the control flow, except for one 
main loop, which iterates reading monitored variables and producing the control variables.

The SCR software specifications can be executed by the existing SCR simulator and tested - either automatically (e.g. random testing) or manually, guided by a domain expert using visual interface mimicking the actual system. The application of testing to the specifications, early in the lifecycle, is more cost effective than testing the actual implementation because removing the errors in the implementation is more expensive.

\subsection{Using Visual Interfaces for Validation}

To date, most validation techniques are highly biased towards calculations involving symbolic representations of problems. These calculations are either formal (in the case of consistency and completeness checks), or informal (in the case of code inspections). We believe that an essential type of "evidence" of the correctness of the formalization process must be provided by human-based calculation, because humans formulate and understand the original problem. We further believe that human calculation can be significantly amplified by shifting from symbolic representation to graphical representations.

Another issue in the formalization phase is the domain expert's understanding of the formal specification. Often the domain expert does not have the expertise in the specific form of formal notation and cannot validate whether the formal specification really captures all desired features of the system correctly.

By using visualization of the system model, it is possible to graphically present the functional capabilities and behavior captured in the formal specification. Using this representation, the correctness of the system model can be validated by human inspection, much in the same way that the mathematically structured formulas are validated by human inspection. Our belief is that human intuition and understanding will be much more effective with respect to a visual representation, than it would be in a 
mathematical domain. The domain experts can interact with the visual interface of the specification and perform tests and simulation runs in order to check if it captures the desired system behavior. Animation of certain important properties can provide further evidence in the correctness of the model [71].

\subsection{Component-based Approach for Verification}

We present a methodology for automated decomposition and abstraction of SCR specifications. The main hypothesis is that components of complex system specifications can be identified at the points of minimal coupling (minimal control and/or information exchange). By applying graph-theoretic min-cut algorithms, we can identify these points and decompose the specification. The smaller components might be amenable to testing and based on the coverage achieved by the executed tests, we explore how to abstract these parts of the specification. The rest of the specification could be exposed to formal analysis, such as model checking or theorem proving. The abstraction reduces the number of states that need to be checked by an automated verification technique and helps in avoiding the state explosion problem, which occurs in traditional model checking.

Automated specification decomposition remains one of the most elusive research goals. An approach to specification decomposition has been presented in [6]. The authors propose an algorithm for slicing system specifications represented with Colored Petri Nets. Slicing the specification improves the understanding of the complex system models and helps with identifying high-risk components early in the life cycle. The underlying ideas of [6] are similar to our approach. We want to decompose complex system specifications into smaller parts with manageable complexity. Instead of ad-hoc decomposition criteria, we propose using minimum coupling. In other words, our approach to decomposition creates system components such that they have minimal coupling (information exchange and/or control connectivity) with the rest of the system.

Several abstraction and slicing criteria for SCR have been proposed in [12]. We demonstrate how min-cut graph algorithms can be used to decompose SCR 
specifications, as well as to automate the specific SCR abstraction method. Proposed methodology provides automated abstraction of "irrelevant" monitored variables and provides guidance on how to perform verification and validation of system models. Consequently, domain and specification experts can focus their formal verification efforts on the identified components, combine the results and provide evidence regarding the correctness of the system as a whole. We present the underlying theory and propose a strategy for verification of decomposable SCR requirements specifications for efficient verification of SCR models.

The main goals of the dissertation are identifying the principles that enable componentbased compositional verification, providing a strategy for verification of decomposable SCR specifications, and demonstrating the advantages of this approach. We also present our ideas on combining different verification methodologies, namely component model checking and random testing, by proposing a property checklist based verification approach.

\subsection{Contributions}

In our research, we have performed the following tasks:

1) Studied different approaches to decompose formal specification models;

2) Investigated the underlying theory for compositional verification of SCR models, which provides sound rules for combination of the component verification results;

3) Investigated the use of visualization approaches for validation and developed techniques for visualization of SCR requirements specifications;

4) Investigated the induced structural coverage by testing of formal models and built tools for measuring the structural coverage of simulations and random testing of software models;

5) Demonstrated the effectiveness of the component-based verification approach for decomposable requirements specifications on a case study. 
This is the set of the original contributions:

1) Development of a divide and conquer methodology for verification and validation of decomposable SCR requirements specifications, which involves testing and formal approaches;

2) Developed a novel decomposition methodology for SCR requirements specifications, based on min-cut graph algorithms, which is used to identify components in the specifications and provide guidance for validation and verification;

3) Identified the underlying principles for writing modular software requirements specifications, which in turn are decomposable;

4) Techniques for visualization of SCR specifications and monitoring of induced structural test coverage.

\subsection{Dissertation Structure}

The dissertation is organized as follows. In Chapter 2 we give a brief overview of the related work. Chapter 3 gives the background on which this dissertation relies and presents a motivational example that is used throughout the exposition. Chapter 4 presents the proposed decomposition methodology and the strategy for component-based verification of high assurance systems. The testing and coverage of software models is presented in Chapter 5. In Chapter 6, we present the ideas on using Visual Interfaces for validation purposes and how different visualization tools can be used together with the SCR toolset. The case study of the NSA Personnel Access Control System SCR specification is given in Chapter 7. Chapter 8 concludes the dissertation giving summary of the accomplishments and providing future directions for research. 


\section{Chapter 2}

\section{Related Work}

Proving the correctness of software programs has been the holy grail of Computer Science and Software Engineering. One of the pioneering works in this area is 1969 C.A.R. Hoare's paper [7] on proving correctness of sequential programs. Hoare presents a formal axiomatic system by which programs can be proved to be correct with respect to assertions about the values of their input and output variables. In order to prove correctness of the program loops in these simple "Pascal" like languages, the verifier must devise a loop invariant. Hoare in [7] states:

“The practice of supplying proofs for nontrivial programs will not become widespread until considerably more powerful proof techniques become available, and even then will not be easy."

Since that time, the complexity of the software we use in everyday lives has escalated, rendering Hoare's method inapplicable. For nearly all programs it is impossible to show that they contain no faults because of their complexity. What we strive for is to show that the probability of latent faults in a software artifact is very low, or the occurrence of failures is highly unlikely. 
Software Reliability as a discipline dates back to the 1970's. In [28] Musa et al. define software reliability as: "the probability of failure-free operation of a computer program for a specified time in a specified environment." Many time-domain models have been proposed for the estimation of software reliability. They use the failure history obtained during testing to predict the actual reliability of the program. The assumption behind these models is that the testing is performed in accordance with a given operational profile. Some of the fundamental problems with these models are the difficulty obtaining the "correct" operational profile, variability of the results based on the assumed operational profile, and the fact that they often overestimate the reliability because of observed saturation in the testing process. Some current approaches in this research field propose incorporating code coverage metrics of the testing process into the reliability model [44][72] in order to avoid some of the mentioned problems. However, for high assurance systems, this approach is not appropriate because the goal of verification is to estimate the reliability of the system independent of the operational profile (even highly unlikely modes of operation should be shown to be highly reliable).

Software testing is used to demonstrate reliability and to detect errors in the developed code. Testing has been used since the early days of programming, and it is still the most commonly used quality assurance technique. It involves generation of test suites and executing the code on them. Testing can be also performed on the specification model, in order to detect errors in the formalization phase. The main disadvantage with software testing is that it is not exhaustive - it helps in detecting program errors but it is likely to miss some of them.

The amount of work that has been done in the area of software verification and validation is overwhelming. In this chapter we will just present related work on which this dissertation is based. Section 2.1. talks about Software Testing. Section 2.2. presents Model Checking and advances in this area like Abstraction and Compositional Methods. Section 2.3. presents results in using theorem proving for software verification. 


\subsection{Software Testing and Coverage Measures}

Usual assumption in the software reliability community is that code coverage achieved by testing is correlated with the reliability of a program [43][53][65]. In practice it has been observed that defect coverage and test coverage grow over time. However, this relationship is complicated [37] and no simple correlation has been found to date. Answering this question is important because it would justify test coverage monitoring for the purposes of reliability estimation.

Different coverage measures have been defined for the purpose of assessing the adequacy of software testing procedures [66][70]. Some of the well known coverage measures are statement, branch and path coverage. Statement coverage is defined as the number of different statements exercised by test cases. Complete statement coverage requires each statement of the code to be executed by at least one test case. Branch coverage is defined by the number of transfer of control (branch) statements executed by the test suite. Complete branch coverage is usually considered to be the minimal testing requirement. It is easy to see that branch coverage subsumes statement coverage, i.e. complete branch coverage implies complete statement coverage. Most comprehensive coverage measure is path coverage, which requires all possible paths in the code to be executed. It subsumes both previous testing strategies (complete statement, and complete branch coverage). Complete path coverage is usually infeasible, because even small programs can have infinite number of paths, primarily because of the loops.

Other coverage measures have been defined to fill in the gap between path coverage and branch coverage. Data flow testing selects paths based on definitions and uses of the variables in the program. A variable is defined when it is assigned a value in a specific statement. Afterwards, every statement that uses the defined variable belongs to the use set. The uses of the variables can be divided in P-uses (predicate uses, branch conditions), or C-uses (computation uses, I/O or calculations involving the variable). Based on definition-clear paths of the variables (a path between a definition and a use of a variable 
in which the variable does not receive a new value in some intermittent node) we can define other coverage measures such as All-uses, All-DU-paths, All-Def, All-C-Some-P, All-P-Some-C, All-P coverage [66]. There are many other coverage measures [80] including: structured path testing, boundary-interior path testing, strategies based on linear code sequence and jump, MC/DC, etc.

Testing strategies are very difficult to compare, primarily because most of them are defined ad-hoc and without theoretical foundations. The usefulness of the "subsumes" relationship between different coverage measures has been highly contested in literature [25][62] observing that most of the strategies are incomparable under subsumption, and it provides no quantitative measure of the effectiveness even for the ordered criteria. Other comparison methodologies have been proposed, such as like using probabilistic comparisons based on randomly selected tests satisfying different criteria [25].

\subsubsection{Specification based Testing}

Weyuker et al. [24] propose several strategies about how to automatically generate test data for a given boolean specification formula. In order to test an implementation of a given boolean formula $F$ with $n$ distinct variables, a number of test cases have to be picked from $n$-dimensional Boolean Space. Exhaustive testing is expensive, because a formula of $n$ variables requires exponential number $-2^{n}$ distinct test cases.

In [24], Weyuker et al. define different categories of test points, the most general being true points (points which cause the formula to evaluate to 1), and false points (points which cause the formula to evaluate to 0 ). True points are furthermore divided on unique true points for particular term $p_{i}$ of the formula given in disjunctive normal form (points which make the particular term true and consequently the whole formula true, however they do not make any other terms true), and overlapping true points (points which are true points for more then one term). The false points are divided on near false points (points which are false for given formula, however they are true points for the same formula with 
one complemented variable in one of the terms - representing incorrect or erroneous implementation) and remaining false points.

The basic strategy is conceived to test for particular type of fault called Variable Negation Fault (one of the variables in the implementation has been mistakenly complemented) and is motivated by hardware testing approaches. It selects one true test

point for each term of the formula, and selects one near false point for each possibly complemented variable in each term of the formula. Refining this basic strategy, Weyuker et al. propose different algorithms for automatic generation of test points for boolean formula specifications. The algorithms differ only in the number of points which are selected from the previously described categories.

\subsubsection{Relation to Dissertation Research}

The proposed algorithms are directly applicable to automated generation of test cases from SCR specifications as well as extending the coverage measures defined in this dissertation. The events and conditions in the SCR model can be represented as boolean formulas and used as input to the proposed algorithms. The test points generated by the algorithms represent particular assignment to the variables involved and used by a model checker to generate test cases for specific transition and the corresponding test points or monitored by the coverage-tracking tool.

Formal approaches to testing have been the focus of recent research. Specification based testing can be used for test generation early in the software lifecycle, before any code is written. Model checking has been used for automated test generation, leveraging the success of model checkers in generating counter examples [4][5]. The advantage of this methodology when compared with random testing has been questioned in a recent study [42]. However, other studies reached the opposite results [45], concluding that more research in this area is required. 


\subsection{Model Checking}

Temporal model checking [22] is a method for automatically deciding if a finite state program satisfies given temporal property. There are two main methods of model checking: state based (ex. the model checker SPIN), using depth-first search algorithm to explore the state space; or symbolic based, using binary decision diagrams (BDD [55]) and breath-first search algorithm to explore the state space (ex. the model checker SMV). An additional model checking method proposed recently is Bounded Model Checking that uses SAT solvers in order to perform model checking with limited depth.

A model checking algorithm for the propositional branching logic CTL was presented at the 1983 POPL conference [21]. The algorithm was linear both in the size of the model determined by the program and in the length of the given temporal property. In the paper, it was used to verify a simple version of the alternating bit protocol with 20 states. Since then, the size of the programs that can be verified by this means has increased dramatically. By developing special programming languages for describing transition systems, it became possible to check examples with several thousand sates. This was sufficient to find subtle errors in a number of nontrivial, although relatively small, protocols and circuit designs [38].

Use of binary decision diagrams [55] led to an even greater increase in size. Representing transition relations implicitly using BDDs made it possible to verify examples that would have required $10^{20}$ states with the original version of the algorithm [33]. Refinements of the BDD-based techniques [32] have pushed the state count up to over $10^{100}$ states. The size of the BDD can be exponential on the length of the formula it represents and is highly dependent on the ordering of the variables. Finding the optimal ordering is NP complete. In practice, symbolic model checking usually gives better results than state based model checking, allowing larger models to be verified.

With recent advances in abstractions [23] the limit has been pushed even further. The authors of [23] report the verification of a pipelined ALU circuit with 64 registers, each 
64 bits wide, having more than $10^{1300}$ reachable states. Another technique that has been proposed to combat the state explosion problem, which plagues model checking, is the compositional verification approach with assume-guarantee reasoning [20][68][69]. Despite the large number of research results in composition, it is rarely adopted in practical software development. Probably the main reasons are the hardness of satisfying the assumptions and that user ingenuity might be required to come up with the decomposition and the needed assumptions.

\subsubsection{Bounded Model Checking}

Bounded Model Checking (BMC) was first proposed by Biere et al. in 1999 [1]. The basic idea is to search for a counterexample in executions whose length is bounded by some integer $k$. If no faults are found then $k$ is increased until a fault is found, we are out of resources, or we have reached some pre-determined upper limit [2].

The BMC problem can be efficiently reduced to a propositional satisfiability problem and can be solved by SAT methods rather than BDDs. Modern SAT solvers can handle propositional satisfiability problems with hundreds of thousands of variables or more. Unfortunately, BMC has the disadvantage of not being complete, in most cases, and consequently not being able to prove absence of errors.

Experiments have shown [2] that if $k$ is small enough, e.g. not more than 60 to 80 steps, BMC outperforms BDD based techniques.

\subsubsection{Relation to Dissertation Research}

Our research addresses the issue of abstraction, but unlike the methods presented in [23], we advocate component-based abstraction, which utilizes the underlying modularity of specifications. We also address the issue of decomposing the specification into selfcontained components by minimizing the information exchange between the components (e.g. joint variables or dependencies) and thus facilitating the ease of compositional validation. 
A recent research addresses these problems by automatically generated invariants which are used for compositional verification [59][60]. Jeffords and Heitmeyer in [59] present the underlying theory for compositional verification of SCR specifications together with a strategy for verification that uses the generated invariants about a single variable and performs abstraction by removing this variable from the specification. Our approach extends this work by considering components of the specification (i.e. sets of variables) which have low coupling in terms of information exchange with the rest of the specification.

We use model checking techniques for verification of the components and present testing approaches applicable in the case when model checking is infeasible. The SCR toolset already has a module for exporting SCR specification in the Promela language which is used by model checker SPIN, as well as a module for exporting models in the language used by the SMV symbolic model checker.

\subsection{Theorem Proving}

Since the early work of Hoare, theorem proving has shown great promise for software verification. It does not suffer from the state explosion problem and even infinite state systems can be verified. However, the main problem with the theorem proving approach is its difficulty of application in practice. Unlike model checking, which is fully automated and represents a "pushbutton technique", theorem proving requires advanced mathematical skills and some user ingenuity in order to guide the theorem proving process.

Hoare's initial work has been later extended by Amir Pnueli and Zohar Manna [73] for the purposes of verification of concurrent and reactive systems. They introduced temporal logic in the computer science arena, and showed how it can be used for reasoning about programs. Interesting work on using temporal logic for compositional specification and verification of concurrent systems can be found in Lamport and Abadi's research papers $[36][41]$. 
Abadi and Lamport argue that the most natural way of representing composition of systems is by using conjunction of their specifications. They developed proof rules for reasoning about the correctness of such systems presented in the formalisms of Temporal Logic of Actions (TLA). This idea of representing the system specification as a conjunction of subsystems is central in the theory underlying the compositional verification of SCR requirements specifications, one of the goals of our research.

One of the most popular mechanized theorem proving systems is the PVS specification and verification system [75]. PVS consists of a specification language, a number of predefined theories, and a theorem prover. The PVS theorem prover provides a collection of powerful primitive inference procedures that are applied interactively under user guidance within a calculus framework. The primitive inferences include propositional and quantifier rules, induction, rewriting, and decision procedures for linear arithmetic.

Current research in the application of theorem proving to software verification is focused on taming the complexity and making it as automated as possible [46].

\subsubsection{Relation to Dissertation Research}

Abadi and Lamport [41] ideas on representing composition of systems by using conjunction of their specifications is central in the underlying theory we present on compositional verification of SCR requirements specifications. Similar to their work, we are also limiting our interest to safety properties, and advocate specifying SCR properties that are associated with components. 


\section{Chapter 3}

\section{Background and a Motivating Example}

In this chapter, we present the research background and tools on which the dissertation relies more heavily, i.e. the SCR requirements methodology and the underlying reachability theory from which the compositional deduction rules arise. We also introduce the min-cut problem by giving a formal problem statement and present some of the current algorithms for its solution. To illustrate the key concepts of this dissertation we present a small SCR requirement specification of a nuclear reactor safety injection system, which was introduced in [50] and is available as an example with the SCR toolset.

\subsection{Software Cost Reduction (SCR) Method}

In SCR we represent the environmental quantities as monitored and controlled variables. The environment non-deterministically produces a sequence of input events, where an input event represents a change in some monitored quantity. The system, represented as a finite state machine, begins execution in an initial state. It responds to each input event by changing the state and, possibly, by producing one or more output events. Output events are changes in controlled variables. An assumption of the model is that at each state transition exactly one monitored variable changes its value, often referred to as "one input" assumption. To concisely capture the system behavior, SCR specifications may 
include two types of internal auxiliary variables: terms, and mode classes. Mode classes and terms often capture historical information.

In the SCR operational model, system $\Sigma$ is defined as a finite state machine $\Sigma=(V, S, \Theta, \rho)$, where $V=\left\{r_{1}, r_{2}, \ldots, r_{n}\right\}$ is the set of state variables, $S$ is the set of states, $\Theta: S \rightarrow$ boolean is the initial state predicate, and $\rho: S \times S \rightarrow$ boolean is the next state predicate representing the transition relation. A state $s$ in $S$ is a function that maps each variable $x \in V$ to a value $x(s)$ from its set of legal values. Usually, the evolution of the system described by the transition relation $\rho$ is deterministic, i.e., it can be represented as a function that maps a given state and an input event to only one possible next state. To construct the next state predicate $\rho$, SCR uses the composition of smaller functions described in a tabular notation, thus improving the readability and understandability of the specification. There are three kinds of tables in SCR requirements specifications, event tables, condition tables, and mode tables. These tables describe the values of each dependent variable, that is, each controlled variable, mode class, or term.

In SCR, a state is a function that maps each variable in the specification to a value, a condition is a predicate defined on a system state, and an event is a predicate defined on a pair of adjacent system states implying that the value of at least one state variable has changed. When a variable changes value, we say that an event "occurs". The following notation is used to denote an event in which some condition becomes true:

$$
@ \mathrm{~T}(c) \stackrel{\operatorname{def}}{=} \neg c \wedge c^{\prime}
$$

where $c$ is a condition evaluated in the current state, and the primed condition is evaluated in the next state. Informally the notation@ @ $(c)$ can be read as "at true c," meaning that we are interested in the event when the logical value of the predicate $c$ changes from false in the current state to true in the next state. Similarly an opposing event is denoted with $@ \mathrm{~F}(c)$ - "at false c", meaning that we are interested in the event when the logic value of the predicate changes from true to false. 


$$
@ \mathrm{~F}(c) \stackrel{\text { def }}{=} @ \mathrm{~T}(\neg c)=c \wedge \neg c^{\prime}
$$

We can also have conditioned events, denoting that we are interested in the event only when some predicate already holds. The expression “@T(c) WHEN $d$ ” represents a conditioned event, which is defined by:

$$
@ \mathrm{~T}(c) \mathrm{WHEN} d \stackrel{\text { def }}{=} \neg c \wedge c^{\prime} \wedge d
$$

where the unprimed conditions $c$ and $d$ are evaluated in the current state and the primed condition $c$ is evaluated in the next state.

The keyword NEVER denotes a special event that is always false. We use the NEVER event in the SCR tables to denote transitions which should never occur during the operation of the system. In addition, there is a special condition keyword INMODE, which is shorthand for specifying that the value of the mode variable is equal to the mode specified in the first column of the row. Similarly, @T(INMODE) denotes the event when the mode variable becomes equal to the mode specified in the first column of the row. We give an example of an SCR requirements specification using all these constructs and keywords in the next section.

A condition table (Table 1) specifies that the value of the variable $X_{i}$ is $v_{k}$ if the boolean condition $c_{j, k}$ holds in mode $m_{j}$.

Table 1: Condition Table format.

\begin{tabular}{|c|c|c|c|c|}
\hline Modes & \multicolumn{4}{|c|}{ Conditions } \\
\hline$m_{1}$ & $c_{1,1}$ & $c_{1,2}$ & $\ldots$ & $c_{1, m}$ \\
\hline$\ldots$ & $\ldots$ & $\ldots$ & $\ldots$ & $\ldots$ \\
\hline$m_{n}$ & $c_{n, 1}$ & $c_{n, 2}$ & $\ldots$ & $c_{n, m}$ \\
\hline$X_{i}$ & $v_{1}$ & $v_{2}$ & $\ldots$ & $v_{m}$ \\
\hline
\end{tabular}

An event table (Table 2) specifies that the variable $Y_{i}$ takes value $v_{k}$ when event $e_{j, k}$ happens in mode $m_{j}$.

Table 2: Event Table format.

\begin{tabular}{|c|c|c|c|c|}
\hline Modes & \multicolumn{4}{|c|}{ Events } \\
\hline$m_{1}$ & $e_{1,1}$ & $e_{1,2}$ & $\ldots$ & $e_{1, m}$ \\
\hline$\ldots$ & $\ldots$ & $\ldots$ & $\ldots$ & $\ldots$ \\
\hline$m_{n}$ & $e_{n, 1}$ & $e_{n, 2}$ & $\ldots$ & $e_{n, m}$ \\
\hline$Y_{i}$ & $v_{1}$ & $v_{2}$ & $\ldots$ & $v_{m}$ \\
\hline
\end{tabular}


Mode tables (Table 3) are a variant of event tables and specify the behavior of a mode class: if mode has value $m_{i}$ and an event $e_{k}$ happens, then mode becomes $m_{j}$.

Table 3: Mode Table format.

\begin{tabular}{|c|c|c|}
\hline Old Mode & Event & New Mode \\
\hline$m_{1}$ & $e_{i}$ & $m_{i}$ \\
\hline$\ldots$ & $\ldots$ & $\ldots$ \\
\hline$m_{n}$ & $e_{j}$ & $m_{k}$ \\
\hline
\end{tabular}

The condition and event tables can have another variant called modeless condition or event tables. These are used in the case when the behavior of a specific variable is not tightly connected to some mode variable, so they have a single row and the mode precondition is considered to be always satisfied.

The SCR model requires the entries in each table to satisfy consistency and completeness properties. The completeness property, in the sense of SCR, is defined as complete definition of the tables, i.e. there cannot be cells which are empty or missing, and each enumerated value must be used as a possible assignment in the corresponding variable specification table. The consistency property ensures that the conditions or events used in specifying the variable are disjoint, i.e. there cannot be non-deterministic assignments of two or more values to a single variable at any point in time. These properties are automatically checked by the SCR toolset and guarantee that all of the tables describe total functions [10]. We must note that the defined completeness and consistency properties do not guarantee the correctness of the developed specification; they just provide assurance in the structural accuracy.

\subsection{Motivating Example - Safety Injection System}

To illustrate the key SCR constructs and the problems we are facing when performing verification we presents a simple specification of a nuclear safety system. The Safety Injection System (SIS) SCR specification describes the safety system of a water coolant system in a nuclear reactor [50]. Based on the pressure sensor readings the system 
decides whether a safety injection of water is needed in the reactor cooling system. The sensor system uses triple modular redundancy to deal with possible sensor failures and erroneous readings. Depending on the pressure monitored by three sensors and using majority voting, the system performs injection if the pressure is too low. Figure 2 presents a visual interface used for simulation and testing of the SIS specification.

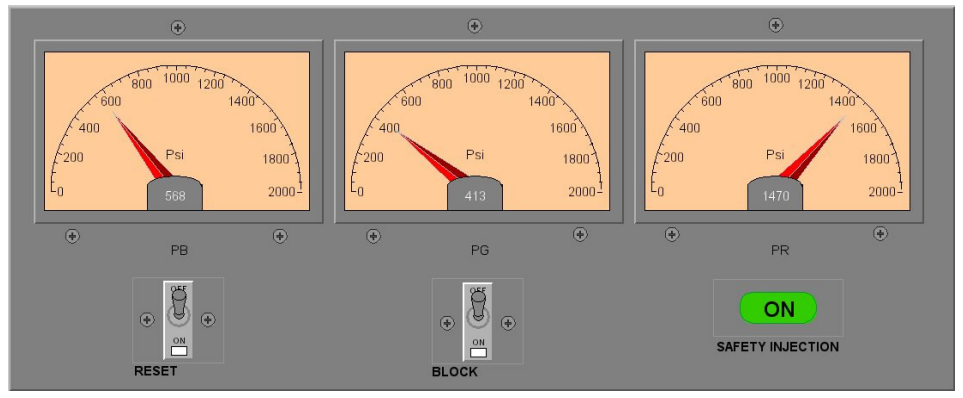

Figure 2: Visual Interface of the SIS specification.

\subsubsection{SCR tables of the SIS specification}

The SIS specification is a small specification with 5 monitored variables, 1 controlled variable, 3 term variables, and 2 mode variables. The SCR specification first defines the variables by giving their types and domains. The pressure sensors PG, PR and PB are monitored variables defined as integers having the range between $[0,2000]$ psi. The other two monitored variables are RESET and BLOCK representing switches on the control table with two possible values $\{\mathrm{ON}, \mathrm{OFF}\}$.

The MajorityPermit term variable is a boolean variable and its specification is given by the condition Table 4. From Table 4 we see that MajorityPermit is set to TRUE when any two pressure sensors are less than the value of the permit constant, which is defined as 1000 psi in the specification document. When we do not have two sensors which are less than permit the MajorityPermit variable is set to FALSE.

Table 4: MajorityPermit Condition Function.

\begin{tabular}{|l|c|c|}
\hline & \multicolumn{2}{|c|}{ Conditions } \\
\hline & $((\mathrm{PG}<$ permit $)$ AND (PR < permit $))$ & NOT $(((\mathrm{PG}<$ permit $)$ AND $(\mathrm{PR}<$ permit $))$ \\
& OR $((\mathrm{PR}<$ permit $)$ AND $(\mathrm{PB}<$ permit $))$ & OR $((\mathrm{PR}<$ permit $)$ AND $(\mathrm{PB}<$ permit $))$ \\
& OR $((\mathrm{PG}<$ permit) AND (PB $<$ permit $))$ & OR $((\mathrm{PG}<$ permit $)$ AND $(\mathrm{PB}<$ permit $)))$ \\
\hline MajorityPermit $=$ & TRUE & FALSE \\
\hline
\end{tabular}


The MajorityLow term variable (Table 5) is specified in an analogous way as the MajorityPermit variable. The only difference is that the low constant is now used, which is defined as 900 psi in the specification document. Table 4 and Table 5 are examples of modeless condition tables, since their definition does not involve a mode variable.

Table 5: MajorityLow Condition Function.

\begin{tabular}{|c|c|c|}
\hline & \multicolumn{2}{|c|}{ Conditions } \\
\hline & $((\mathrm{PG}<$ low $)$ AND $(\mathrm{PR}<$ low $))$ & NOT $(((\mathrm{PG}<$ low $)$ AND $(\mathrm{PR}<$ low $))$ \\
& OR $((\mathrm{PR}<$ low $)$ AND $(\mathrm{PB}<$ low $))$ & OR $((\mathrm{PR}<$ low $)$ AND $(\mathrm{PB}<$ low $))$ \\
& OR $((\mathrm{PG}<$ low $)$ AND $(\mathrm{PB}<$ low $))$ & OR $((\mathrm{PG}<$ low $)$ AND $(\mathrm{PB}<$ low $)))$ \\
\hline MajorityLow $=$ & TRUE & FALSE \\
\hline
\end{tabular}

Table 6: PermitStatus Mode Transition Function.

\begin{tabular}{|c|c|c|}
\hline Source Mode(s) & Events & Destination Mode \\
\hline BelowPermissive & @F(MajorityPermit) & AbovePermissive \\
\hline AbovePermissive & @T(MajorityPermit) & BelowPermissive \\
\hline
\end{tabular}

Table 6 presents the mode table for the PermitStatus mode variable. The first row of the table specifies that if the previous value of the PermitStatus is BelowPermissive and the boolean variable MajorityPermit becomes false then PermitStatus is assigned AbovePermissive as the new value. Similarly, the second row states that if the previous value was AbovePermissive and MajorityPermit becomes true, the next value of PermitStatus will be BelowPermissive. So, the assignment of a new value to a mode variable depends on its previous value, and an event causing the transition.

Table 7: M_Pressure Mode Transition Function.

\begin{tabular}{|c|c|c|}
\hline Source Mode(s) & Events & Destination Mode \\
\hline Low & @F(MajorityLow) & Normal \\
\hline Low & @F(MajorityPermit) & VoterFailure \\
\hline Normal & @T(MajorityLow) WHEN MajorityPermit & Low \\
\hline Normal & $@ T(M a j o r i t y L o w)$ WHEN (NOT MajorityPermit) & VoterFailure \\
\hline VoterFailure & $@ T(M a j o r i t y P e r m i t)$ & Low \\
\hline VoterFailure & @F(MajorityLow) & Normal \\
\hline
\end{tabular}


The mode variable M_Pressure is defined by the function described in Table 7. The table specifies the following transitions for the M_Pressure variable: 1) in case the current mode is Low and the MajorityLow variable becomes false, then the new mode for M_Pressure will be Normal; 2) in case the current mode is Low and the MajorityPermit variable becomes false, then the new mode for M_Pressure will be VoterFailure; 3) if the mode is Normal and MajorityLow becomes true when MajorityPermit is true, then the next value for the M_Pressure will be Low; 4) if the current mode is Normal and the MajorityLow variable becomes true when MajorityPermit is not true, then the new mode will be VoterFailure; 5) if the current mode is VoterFailure and the MajorityPermit variable becomes true then the new mode for M_Pressure will be low; and 6) if the current mode is VoterFailure and the MajorityLow variable becomes false, then the new mode will be Normal.

Table 8: OVERRIDDEN Event Function.

\begin{tabular}{|c|c|c|}
\hline Modes for PermitStatus & \multicolumn{2}{|c|}{ Events } \\
\hline AbovePermissive & Never & @T(Inmode) \\
\hline BelowPermissive & @T(Block = ON) WHEN (Reset = OFF) & @T(Reset = ON) \\
\hline BelowPermissive & Never & @T(Inmode) \\
\hline OVERRIDDEN' $=$ & TRUE & FALSE \\
\hline
\end{tabular}

The OVERRIDEN term variable is specified by the regular SCR event table given in Table 8. Depending on the current value of the PermitStatus mode variable, the new value of OVERRIDEN is assigned to TRUE or FALSE. In essence, OVERRIDEN becomes TRUE only if PermitStatus is BelowPermissive and the monitored variable Block becomes equal to ON when the Reset variable is equal to OFF. Whenever PermitStatus becomes equal to AbovePermissive or BelowPermisive, or in the event that the Reset variable becomes equal to $\mathrm{ON}$ when the PermitStatus is BelowPermissive, the OVERRIDEN variable becomes equal to FALSE. 
Table 9: Safety_Injection Condition Function.

\begin{tabular}{|c|c|c|}
\hline Modes for M_Pressure & \multicolumn{2}{|c|}{ Conditions } \\
\hline Normal & FALSE & TRUE \\
\hline Low & NOT OVERRIDDEN & OVERRIDDEN \\
\hline VoterFailure & TRUE & FALSE \\
\hline Safety_Injection $=$ & ON & OFF \\
\hline
\end{tabular}

The controlled variable Safety_Injection (Table 9) determines whether a safety injection is performed or not depending on the current value of the M_Pressure mode variable. When M_Pressure is Normal, the value of the Safety_Injection variable is OFF; when the M_Pressure is Low and the OVERRIDEN variable is FALSE, the Safety_Injection is ON, otherwise in the same mode when the OVERRIDEN variable is TRUE the Safety_Injection is OFF; finally if the mode of M_Pressure is VoterFailure then Safety_Injection is ON.

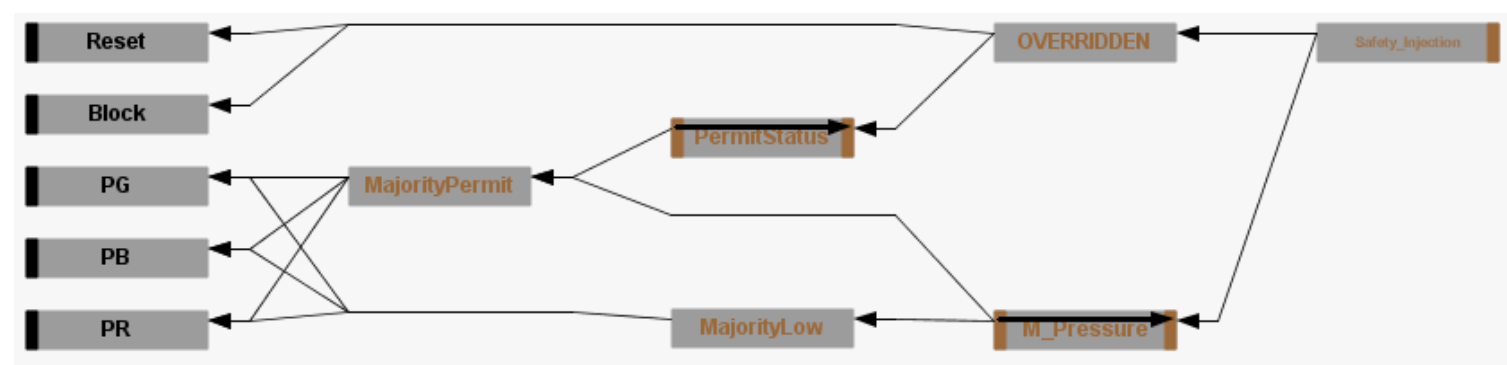

Figure 3: Dependency Graph of the Safety Injection SCR specification.

Given the SCR specification in tabular notation, we can observe that there are dependencies between the variables (Figure 3). In other words, the value of a particular variable depends on values of some other variables. This dependency chain starts with the monitored variables, used by the system to observe changes in the environment, and ends with controlled variables, which are produced by the system in order to affect the environment. The SCR toolset [9] includes dependency graph browser, which is used for navigation through or manual slicing of the specification under development. 


\subsubsection{Problems in the Verification}

Once the operational part of the SIS is specified, we proceed with verification of the stated properties that the specification must satisfy. We are focusing on safety properties, which can be either state or transition properties.

For example, we would like to prove that the following property holds:

Override Works: Reset $=O N=>$ not OVERRIDDEN.

This property states that the system cannot be stuck in overridden mode, i.e., if the monitored variable Reset is ON, then Overriden should be false.

pan: out of memory

(Spin Version 4.2.5 -- 2 April 2005)

Warning: Search not completed

Full statespace search for:

never claim - $\quad$ (not selected)

assertion violations +

cycle checks - (disabled by -DSAFETY)

invalid end states +

State-vector 40 byte, depth reached 499999, errors: 0

$1.1879 \mathrm{e}+07$ states, stored

$2.2326 \mathrm{e}+06$ states, matched

1.41116e+07 transitions (= stored+matched)

0 atomic steps

hash conflicts: $1.92053 \mathrm{e}+06$ (resolved)

Stats on memory usage (in Megabytes):

522.675 equivalent memory usage for states (stored*(State-vector + overhead))

475.349 actual memory usage for states (compression: $90.95 \%$ )

State-vector as stored $=36$ byte +4 byte overhead

33.554 memory used for hash table (-w23)

14.000 memory used for DFS stack (-m500000)

27.836 other (proc and chan stacks)

0.082 memory lost to fragmentation

536.822 total actual memory usage

Figure 4: Execution of the SPIN on the complete model. 
We first tried to run the model checker SPIN on the whole model. As expected, this was unsuccessful because we have run out of memory (see Figure 4). Since PG, PB, PR are integer variables with a big range we observed state space explosion when using explicit model checkers like SPIN. The application of the symbolic model checking tool SMV was successful and proved the property in 16 seconds. We also tried to apply the inductive theorem proving tool SALSA, however, it was unsuccessful in proving the property because of the incompleteness of the tool.

\subsubsection{Proposed Approach}

We explore how to utilize the modular structure of the SIS specification in order to enable more efficient verification of the stated properties. For example, the SIS specification can be decomposed into two parts: the triple modular redundancy sensor module (lower left corner of Figure 5), and the control module (upper part in Figure 5). The properties that involve variables within a single component can be soundly verified by abstracting the rest of the system. The properties that involve variables within several components can be verified either by combining the affected components or by using compositional proof rules.

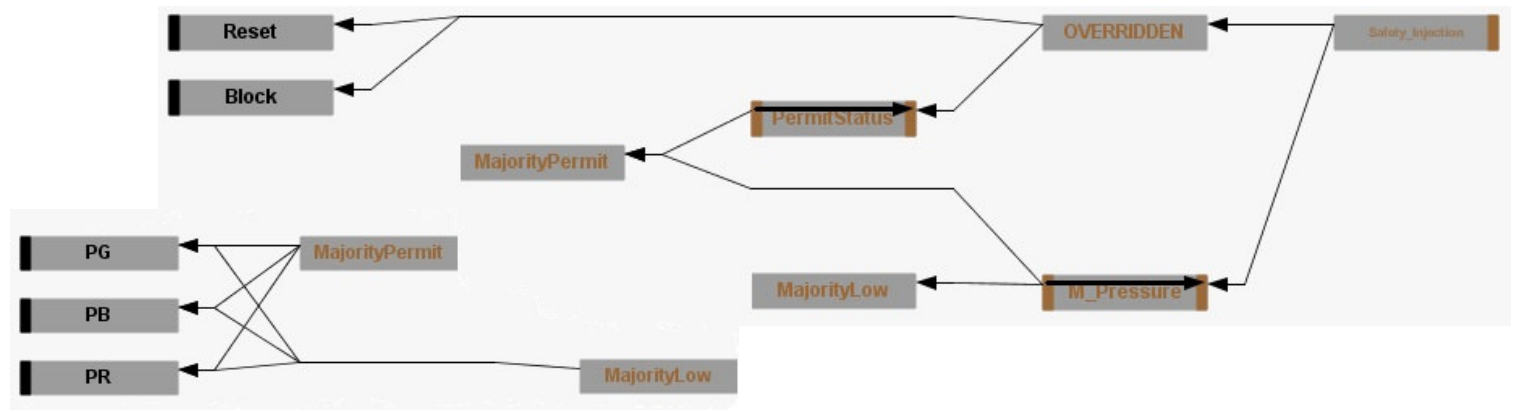

Figure 5: Decomposition of the SIS specification. 
In order to verify the stated Override Works property, we observe that we can focus on the identified control module. Application of the SPIN model checker verifies the property in 0.062 seconds avoiding the state explosion problem. SMV also verifies the property in 0.016 seconds, which is 1000 times faster than performing verification on the complete system.

We just demonstrated a single property, which is not enough to establish the correctness of the whole system (i.e. our checklist representing required properties is far from complete). However, we see that being able to verify properties focusing just on components of the specification is beneficial from performance standpoint. We must note that in general this is not as easy as illustrated in the given example. The underlying assumptions for this methodology to work are that the original specification is modular in nature and that the properties have been stated on component boundaries, or they involve only few components. Emergent system properties should be presented as conjunction of component properties to ease their compositional verification. We present a decomposition methodology that identifies the components of a given SCR specification and present a strategy for efficient verification of decomposable specifications.

\subsection{Theoretical Basis for the Verification of Decomposable Models}

In this section, we review the theoretical foundation for the compositional verification of SCR requirements specifications based on [59]. As mentioned previously, the SCR model of a given system $\Sigma$ can be viewed as a finite state machine, represented by the quadruple $\Sigma=(V, S, \Theta, \rho)$ where:

- $\quad V$ is a set of variables. It contains the monitored, controlled, and internal (term and mode class) variables of the system;

- $S$ is the set of system states. Each state $s \in S$ maps each variable $x \in V$ to a value in its set of legal values. The value of a variable $x$ in state $s$ is denoted by $x(s)$;

- $\Theta: S \rightarrow$ boolean is a one-state predicate defining the set of initial states; 
- $\rho: S \times S \rightarrow$ boolean is a two-state predicate defining the transitions of $\Sigma$. A state $s$ may transition to a sate $s^{\prime}$ if $\rho\left(s, s^{\prime}\right)$ is true.

The properties the system must satisfy, called system invariants, are represented by the first order logical formulae (predicates), defined on a single state, or a transition. The truth value of a single state predicate $\phi(s)$ is calculated by replacing the variables it involves with the values from $s$. Two-state predicate $\varphi\left(s, s^{\prime}\right)$ is evaluated with values from $s$ replacing unprimed variables and values from $s$ ' replacing primed variables. The following are standard definitions for reachability and invariants (e.g. see [54]).

Definition 1: Given a state machine $\Sigma=(V, S, \Theta, \rho)$, a state $s \in S$ is reachable, denoted $\operatorname{Reachable}_{\Sigma}(s)$, if and only if it is one of the initial states or if there exists another reachable state $s_{1}$ from which we can make a transition to the state $s$.

$$
\operatorname{Reachable}_{\Sigma}(s) \Leftrightarrow \Theta(s) \vee \exists s_{1} \in S: \operatorname{Reachable}_{\Sigma}\left(s_{1}\right) \wedge \rho\left(s_{1}, s\right)
$$

Often we need to use induction on the number of steps, so the following definition is beneficial. A state $s \in S$ reachable in $n$ steps denoted Reachable ${ }_{\Sigma}^{n}(s)$ is defined by:

$$
\text { Reachable }_{\Sigma}{ }_{\Sigma}(s) \Leftrightarrow\left\{\begin{array}{l}
\Theta(s), n=0 \\
\exists s_{1} \in S: \text { Reachable }{ }_{\Sigma}^{n-1}\left(s_{1}\right) \wedge \rho\left(s_{1}, s\right), n>0
\end{array}\right.
$$

Definition 2: Given a state machine $\Sigma=(V, S, \Theta, \rho)$, a one-state predicate $\phi(s)$ is a state invariant of $\Sigma$ if and only if it holds for all reachable states.

$$
\phi \in \operatorname{Inv}(\Sigma) \Leftrightarrow \forall s \in S: \operatorname{Reachable}_{\Sigma}(s) \Rightarrow \phi(s)
$$


Definition 3: A two-state predicate $\varphi\left(s, s^{\prime}\right)$ is a transition invariant of $\Sigma$ if and only if it holds for all pairs of adjacent reachable states.

$$
\varphi \in \operatorname{Inv}(\Sigma) \Leftrightarrow \forall s, s^{\prime} \in S:\left(\operatorname{Reachable}_{\Sigma}(s) \wedge \rho\left(s, s^{\prime}\right)\right) \Rightarrow \varphi\left(s, s^{\prime}\right)
$$

The following theorems (Theorem 1 and 2) are also given in [54].

Theorem 1: Let $\Sigma=(V, S, \Theta, \rho)$, then $\phi(s)$ is a state invariant of $\Sigma$ if the following holds:

$$
(\forall s \in S: \Theta(s) \Rightarrow \phi(s)) \wedge\left(\forall s, s^{\prime} \in S: \phi(s) \wedge \rho\left(s, s^{\prime}\right) \Rightarrow \phi\left(s^{\prime}\right)\right)
$$

The proof follows from definitions 1 and 2, by using induction on the number of steps. Basically we ensure that the property $\phi(s)$ holds for the initial states, and then show that if it holds in the current state, it must also hold in the next state defined by the transition relation, thus covering all reachable states as required by definition 2 . However, it covers more than just the reachable states, thus causing incompleteness. Some unreachable states can cause the right side of the premise to fail and dispute the validity of some probable invariant.

This theorem gives us the initial deductive rule for proving single state invariants (Eq. 1). In this shorter notation $\phi$ is a single state property and $\phi$ denotes the same property evaluated in the next state.

$$
\frac{\Theta \Rightarrow \phi,(\phi \wedge \rho) \Rightarrow \phi^{\prime}}{\phi \in \operatorname{Inv}(\Sigma)} \text { (Eq. 1) }
$$

We can strengthen the rule by using additional previously proven invariants. This allows us to reduce the incompleteness in the previous rule by using invariants that restrict the unreachable states causing it to fail. The deduction rules (Eq. 2) are obtained, where $\alpha$ represents a single state invariant and $\beta$ represents a transition invariant for the system $\Sigma$. 


$$
\begin{gathered}
\frac{\alpha \in \operatorname{Inv}(\Sigma),(\alpha \wedge \Theta) \Rightarrow \phi,\left(\phi \wedge \alpha \wedge \alpha^{\prime} \wedge \rho\right) \Rightarrow \phi^{\prime}}{\phi \in \operatorname{Inv}(\Sigma)} \\
\text { and } \left.\frac{\beta \in \operatorname{Inv}(\Sigma), \Theta \Rightarrow \phi,(\phi \wedge \beta \wedge \rho) \Rightarrow \phi^{\prime}}{\phi \in \operatorname{Inv}(\Sigma)} \text { (Eq. } 2\right)
\end{gathered}
$$

Theorem 2: Let $\Sigma=(V, S, \Theta, \rho)$, then $\varphi\left(s, s^{\prime}\right)$ is a transition invariant of $\Sigma$ if the following holds: $\forall s, s^{\prime} \in S: \rho\left(s, s^{\prime}\right) \Rightarrow \varphi\left(s, s^{\prime}\right)$.

The proof follows from definition 3 by removing the requirement that the state $s$ should be reachable. This introduces incompleteness, meaning that there might be invariants that we might not be able to prove by this theorem.

Like in the previous case, this theorem gives us the initial deduction rule (Eq. 3) for proving transition invariants.

$$
\frac{\rho \Rightarrow \varphi}{\varphi \in \operatorname{Inv}(\Sigma)}(\text { Eq. 3) }
$$

It also can be strengthened by using auxiliary state or transition invariants as presented in (Eq. 4).

$$
\frac{\alpha \in \operatorname{Inv}(\Sigma),\left(\alpha \wedge \alpha^{\prime} \wedge \rho\right) \Rightarrow \varphi}{\varphi \in \operatorname{Inv}(\Sigma)} ; \text { and } \frac{\beta \in \operatorname{Inv}(\Sigma),(\beta \wedge \rho) \Rightarrow \varphi}{\varphi \in \operatorname{Inv}(\Sigma)} \text { (Eq. 4) }
$$

The problem in using these rules for verification of system properties is that they rely on the specification of entire system $\Sigma=(V, S, \Theta, \rho)$. We would like to apply our decomposition methodology to divide the system into smaller subsystems $\Sigma_{1}, \Sigma_{2}, \ldots, \Sigma_{n}$, and perform the verification task on these components instead on the complete system. 


\subsubsection{Compositional Verification Rules}

Abadi and Lamport [41] argue that most natural way of representing composition of systems is by using conjunction of their specifications. This idea of representing the system specification as conjunction of subsystems is central in the underlying theory we present on compositional verification of SCR requirements specifications. Similar to their work, we are also limiting our interest to safety properties, and advocate specifying SCR properties that are associated with components.

Without the loss of generality, we consider how to decompose a given system $\Sigma=(V, S, \Theta, \rho)$ into two subsystems $\Sigma_{1}$ and $\Sigma_{2}$ allowing us to perform verification on the components. We use the same definition of parallel composition of state machines as given by Jeffords and Heitmeyer in [59].

Definition 4: Given two state machines $\Sigma_{1}=\left(V, S, \Theta_{1}, \rho_{1}\right)$ and $\Sigma_{2}=\left(V, S, \Theta_{2}, \rho_{2}\right)$, with the same set of variables and the same allowed values for each of the variables, their parallel composition $\Sigma_{1} \| \Sigma_{2}$ is defined as conjunction, i.e., $\Sigma_{1} \| \Sigma_{2}=\left(V, S, \Theta_{1} \wedge \Theta_{2}, \rho_{1} \wedge \rho_{2}\right)$.

Theorem 3: All reachable states within $\mathrm{n}$ steps of $\Sigma_{1} \| \Sigma_{2}$ are those that are reachable in both $\Sigma_{1}$ and $\Sigma_{2}$ within n steps.

$$
\operatorname{Reachable}_{\Sigma 1 \| \Sigma 2}^{n}(s) \Leftrightarrow \text { Reachable }_{\Sigma 1}^{n}(s) \wedge \text { Reachable }_{\Sigma 2}^{n}(s)
$$

The proof follows from definitions 1 and 4 using induction on the number of steps needed to reach the state. Since the set of all reachable states is obtained when we let the number of steps to go to infinity

$$
\left\{\mathrm{s}: \mathrm{s} \in \mathrm{S} \wedge \operatorname{Reachable}_{\Sigma}(\mathrm{s})\right\}=\left\{\mathrm{s}: \mathrm{s} \in \mathrm{S} \wedge \operatorname{Reachable}_{\Sigma}^{n \rightarrow \infty}(\mathrm{s})\right\}
$$


we conclude that all reachable states of $\Sigma_{1} \| \Sigma_{2}$ are those that are reachable in both $\Sigma_{1}$ and $\Sigma_{2}$

Corollary 1: Invariants of each of the subsystems are also invariants of the composition, i.e., $\operatorname{Inv}\left(\Sigma_{i}\right) \subseteq \operatorname{Inv}\left(\Sigma_{1} \| \Sigma_{2}\right)$ for $i=1,2$. From Theorem 3 it follows that each component contains all reachable states of the composition and possibly some more. Although this seams contradictory for verification purposes, we will show in Chapter 4 that focusing the verification on properties that are stated on components boundaries and performing abstractions will reduce the state spaces of the derived components.

Since the components may have additional reachable states, possibly invalidating properties that might hold true, we can use already proven invariants from the other components to strengthen the deduction rules. The rules (Eq. 5) can be used for this purpose, where $\alpha$ represents a single state invariant and $\beta$ represents a transition invariant for the subsystem $\Sigma_{1}$.

$$
\begin{aligned}
& \frac{\alpha \in \operatorname{Inv}\left(\Sigma_{1}\right),\left(\alpha \wedge \Theta_{2}\right) \Rightarrow \phi,\left(\phi \wedge \alpha \wedge \alpha^{\prime} \wedge \rho_{2}\right) \Rightarrow \phi^{\prime}}{\phi \in \operatorname{Inv}\left(\Sigma_{1} \| \Sigma_{2}\right)} \\
& \text { and } \frac{\beta \in \operatorname{Inv}\left(\Sigma_{1}\right), \Theta_{2} \Rightarrow \phi,\left(\phi \wedge \beta \wedge \rho_{2}\right) \Rightarrow \phi^{\prime}}{\phi \in \operatorname{Inv}\left(\Sigma_{1} \| \Sigma_{2}\right)} \text { (Eq. 5) }
\end{aligned}
$$

A similar sound compositional rule for proving a two-state property $\varphi$ is invariant is given in (Eq. 6):

$$
\frac{\alpha \in \operatorname{Inv}\left(\Sigma_{1}\right),\left(\alpha \wedge \alpha^{\prime} \wedge \rho_{2}\right) \Rightarrow \varphi}{\varphi \in \operatorname{Inv}\left(\Sigma_{1} \| \Sigma_{2}\right)} ; \text { and } \frac{\beta \in \operatorname{Inv}\left(\Sigma_{1}\right),\left(\beta \wedge \rho_{2}\right) \Rightarrow \varphi}{\varphi \in \operatorname{Inv}\left(\Sigma_{1} \| \Sigma_{2}\right)} \text { (Eq. 6) }
$$

Thus, the strategy for verification would be to decompose a given system into components which if composed using parallel composition result in the system itself. 
Then we prove invariants that hold for the components by using the deduction rules. From the Corollary 1 it follows that the proven invariants also hold for the complete system. Jeffords and Heitmeyer in [59] present a strategy for verification that performs abstraction by removing a single variable from the specification and uses the automatically generated invariants [60] for the abstracted variable to strengthen the deduction rules. Our approach extends this strategy by considering components of the specification (i.e. sets of variables) which have low coupling and information exchange with the rest of the specification. Instead of automatically generating invariants for the abstracted components, our approach is to specify properties on component level and later try to prove that they are invariants and use them in the certification process of the system as a whole.

Since each component usually assumes some properties about the environment for its correct operation, the rules (Eq. 5) and (Eq. 6) can be considered as assume-guarantee rules. We use them to prove and guarantee some properties of the component and consequently the system based on assumptions about the environment. These assumptions are either properties stating the correctness of components that the specific component interacts with, or assumptions about the monitored variables.

\subsubsection{Circular Assume-Guarantee Rules}

McMillan [34] argues that often in practical applications there is a need for a circular assume-guarantee rule. This happens when some properties $\phi_{1}$ and $\phi_{2}$, which we want to prove, require that the proof of $\phi_{1}$ for subsystem $\Sigma_{1}$ depends on $\phi_{2}$ and that the proof of $\phi_{2}$ for subsystem $\Sigma_{2}$ depends on $\phi_{1}$. During our research, we have not witnessed a case where such a rule was needed or applicable on SCR requirements we worked with. This is probably due to our restriction to state and transition safety properties, which are most commonly used in SCR specifications. In the case when more complex temporal properties are considered, we envision that this rule comes into play. Its exposition is useful since, as demonstrated by Jeffords and Heitmeyer [59], it leads to compositional rules similar to the ones demonstrated in the previous section that are geared towards model checking. 
McMillan's rule for circular compositional reasoning is given by (Eq. 7). The basic idea is to use induction and show that if assuming $\phi_{2}$ holds during the evolution of the system $\Sigma_{1}$ from step 0 to $n-1$, then $\phi_{1}$ also holds from 0 up to step $n$. If the opposite is demonstrated for the system $\Sigma_{2}$, proving that $\phi_{2}$ holds from 0 up to step $n$ assuming that $\phi_{1}$ holds from 0 to $n-1$, then by induction we conclude that both properties hold for the system. In general, McMillan's rule is used for proving the invariance of conjunction of two Linear Temporal Logic (LTL) properties $\phi_{1} \wedge \phi_{2}$, but in our case we are only interested in the defined state and transition properties.

$$
\begin{aligned}
& \Sigma_{1} \mid=\phi_{2} \text { up to } n-1 \Rightarrow \phi_{1} \text { up to } n \\
& \frac{\Sigma_{2} \mid=\phi_{1} \text { up to } n-1 \Rightarrow \phi_{2} \text { up to } n}{\phi_{1} \wedge \phi_{2} \in \operatorname{Inv}\left(\Sigma_{1} \| \Sigma_{2}\right)} \text { (Eq. 7) }
\end{aligned}
$$

For the special case when $\phi_{1}$ and $\phi_{2}$ are state properties, the McMillan rule can be simplified using the following observation. The fact that one-state property $\phi$ holds up through step $n-1$ can be soundly replaced by the conjunction of the assumed one-state property with both $\Theta$ and $\rho$ in $\Sigma=(V, S, \Theta, \rho)$, producing a new system $\Pi=(V, S, \phi \wedge \Theta, \phi \wedge \rho)$. This simplification with the additional requirement that $\phi_{1}$ and $\phi_{2}$ hold for the initial states of $\Sigma_{1} \| \Sigma_{2}$ produces the following circular assume-guarantee rule:

$$
\frac{\Theta_{1} \wedge \Theta_{2} \Rightarrow \phi_{1} \wedge \phi_{2}, \phi_{1} \in \operatorname{Inv}\left(V, S, \phi_{2} \wedge \Theta_{1}, \phi_{2} \wedge \rho_{1}\right), \phi_{2} \in \operatorname{Inv}\left(V, S, \phi_{1} \wedge \Theta_{2}, \phi_{1} \wedge \rho_{2}\right)}{\phi_{1} \wedge \phi_{2} \in \operatorname{Inv}\left(\Sigma_{1} \| \Sigma_{2}\right)}
$$

This proof rule also can be strengthened by adding auxiliary state invariants, $\alpha \in \operatorname{Inv}\left(\Sigma_{1}\right)$ and $\beta \in \operatorname{Inv}\left(\Sigma_{2}\right)$ to improve the completeness of the rule:

$$
\begin{gathered}
\alpha \in \operatorname{Inv}\left(\Sigma_{1}\right), \beta \in \operatorname{Inv}\left(\Sigma_{2}\right), \Theta_{1} \wedge \Theta_{2} \Rightarrow \phi_{1} \wedge \phi_{2}, \\
\phi_{1} \in \operatorname{Inv}\left(V, S, \alpha \wedge \beta \wedge \phi_{2} \wedge \Theta_{1}, \alpha \wedge \beta \wedge \alpha^{\prime} \wedge \beta^{\prime} \wedge \phi_{2} \wedge \rho_{1}\right), \\
\frac{\phi_{2} \in \operatorname{Inv}\left(V, S, \alpha \wedge \beta \wedge \phi_{1} \wedge \Theta_{2}, \alpha \wedge \beta \wedge \alpha^{\prime} \wedge \beta^{\prime} \wedge \phi_{1} \wedge \rho_{2}\right)}{\phi_{1} \wedge \phi_{2} \in \operatorname{Inv}\left(\Sigma_{1} \| \Sigma_{2}\right)} . \text { (Eq. 9) }
\end{gathered}
$$


For the special case when $\beta=$ TRUE and $\phi_{1}=$ TRUE, by renaming $\phi_{2}$ to $\phi$ we obtain rule (Eq. 10) which similar to (Eq. 5). The rule (Eq. 10) is more applicable to model checking or any other method of establishing invariants, instead of using proofs of implications required by rule (Eq. 5).

$$
\frac{\alpha \in \operatorname{Inv}\left(\Sigma_{1}\right), \Theta_{1} \wedge \Theta_{2} \Rightarrow \phi, \phi \in \operatorname{Inv}\left(V, S, \alpha \wedge \Theta_{2}, \alpha \wedge \alpha^{\prime} \wedge \rho_{2}\right)}{\phi \in \operatorname{Inv}\left(\Sigma_{1} \| \Sigma_{2}\right)} \text { (Eq. 10) }
$$

Applying rule (Eq. 1) we arrive at the exact version of rule (Eq. 5), but now starting from the McMillan's assume guarantee rule:

$$
\frac{\alpha \in \operatorname{Inv}\left(\Sigma_{1}\right),\left(\alpha \wedge \Theta_{2}\right) \Rightarrow \phi,\left(\phi \wedge \alpha \wedge \alpha^{\prime} \wedge \rho_{2}\right) \Rightarrow \phi^{\prime}}{\phi \in \operatorname{Inv}\left(\Sigma_{1} \| \Sigma_{2}\right)}
$$

\subsection{The Min-Cut problem}

We propose to use min-cut graph algorithms to determine the points of minimal coupling in a given SCR specification thus automating the component identification and abstraction.

The minimum cut problem is defined as follows:

Given an undirected graph $G(E, V)$ with a set of vertices $|V|=n$ and a set of corresponding edges $|E|=m$, we are interested in partitioning the vertices in two sets, $V_{1}$ and $V_{2}$, such that the sum of the weights of the edges with end-points in the different sets is minimal.

A closely related problem to the general minimum cut is finding the minimum capacity cut between two given vertices, source - $s$ and a sink - $t$, called the minimum $s$ - $t$ cut problem. Thanks to the maximum flow - minimum cut theorem, maximum flow algorithms can be used to identify the minimum $s$ - $t$ cut in polynomial time. 


\section{The maximum flow - minimum cut theorem [35][64] states:}

The maximal flow between two vertices $v_{i}$ and $v_{j}$ in a graph $G$ is exactly the weight of the smallest set of edges to disconnect $G$ with $v_{i}$ and $v_{j}$ in different components.

Given an undirected graph $G(E, V)$ with a set of vertices $|V|=n$ and a set of corresponding edges $|E|=m$, the classical algorithms of Ford and Fulkerson [35] and Edmonds and Karp [29], solve the maximum flow in $O\left(n^{3}\right)$ time. For extensive review on the minimum cut algorithms and their performance information, the reader is referred to [8]. We used the author's implementations, which are freely available [76], to conduct the research presented in this dissertation. The fastest current algorithms for the minimum $s$ - $t$ cut problem use flow techniques, in particular, the push-relabel method and run in $\omega(\mathrm{nm})$ time. For the general minimum cut problem - the classical Gomory-Hu algorithm solves the minimum cut problem using $(n-1)$ minimum $s$ - $t$ cut computations.

Recent advances resulted in faster algorithms for the minimum cut problem. Hao and Orlin developed an algorithm based on the push-relabel method, which shows how to perform all $(n-1)$ minimum $s-t$ cuts in time asymptotically equal to that needed to perform one $s$ - $t$ minimum cut computation. This algorithm runs in $O\left(n m \log \left(n^{2} / m\right)\right)$ time. Several other algorithms for the general min cut problem have been proposed that have better bounds than the known algorithms for the minimum $s-t$ cut problem. The algorithm of Nagamochi and Ibaraki runs in $O(n(m+n \log n))$ time. The algorithm of Karger and Stein runs in $O\left(n^{2} \log ^{3} n\right)$ expected time. Two closely related algorithms of Karger run in $O\left(m \log ^{3} n\right)$ and $O\left(n^{2} \log n\right)$ expected time. 


\section{Chapter 4}

\section{Component-based Verification}

Complex systems typically contain subsystems, which include different and possibly disjoint sets of monitored variables. For example, in an avionic system we have a navigation subsystem that depends on the altitude, speed, and direction; another lifesupport subsystem would deal with the cabin pressure and temperature. Several specification slicing techniques [6][12] have been proposed in order to tame a system's complexity and avoid state space explosion in formal verification. However, most of them are done ad hoc and require a user's experience in order to be applied successfully. Validation and verification of such complex specifications can be extremely timeconsuming, especially if we are not familiar with the system being specified.

Since the early days of the SCR Method, Parnas et al. [15] have argued that complex systems must be built by utilizing a modular structure. They demonstrate how the information hiding and abstraction principles are to be followed in the design of the Onboard Flight Program (OFP) for the A-7E aircraft by writing a hierarchical module guide document. The guide is intended to achieve the following goals:

a) A software engineer should be able to understand the responsibility of a module without understanding the module's internal design; 
b) A reader with a well-defined concern should easily be able to identify the relevant modules without studying irrelevant modules;

c) The number of branches at each non-terminal module in the hierarchy graph should be small enough so that the designers can prepare convincing arguments demonstrating that the sub-modules have no overlapping responsibilities and that the module covers all of the intended responsibilities.

These principles, since their introduction, have lead to the development of the Object Oriented programming methodologies. We argue that the same principles must be followed while writing the operational part (SCR tables) of any large SCR specification. The responsibilities of the components should be stated by the property-based part of the specification, creating a checklist for verification and validation of the components and the system as a whole. The verification process is simplified if the properties are stated on component boundaries (involving only the variables within a single component), as advocated by Abadi and Lamport [41].

Given an SCR specification in tabular notation, dependencies between the variables can be observed easily. Dependency chains end with controlled variables, which are generated by the system and affect the environment, and start with the monitored variables, which are used by the system to observe the changes in the environment. How the monitored variables are used, and how the controlled variables are produced is formally defined by the SCR specification and its constructs: tables, term variables, mode class variables, etc.

Our hypothesis is that if a given SCR model has modular structure as advocated by Parnas et al. in [15], then the boundaries of different subsystems in complex SCR specifications can be automatically identified at the points of minimal coupling with the rest of the specification. The expectation is that the subsystems generally transform monitored variables into a smaller set of derived variables (term variables), which are then further utilized to calculate the values of controlled variables. 
We use min-cut graph algorithms [8] on the dependency graphs to identify these points of minimal coupling and decompose the system in smaller components. The overarching idea is to apply a divide and conquer approach in the verification of SCR specifications. The min-cuts result in partitioning of the set of variables. Each partition represents a smaller component of the specification. Only the properties that involve the variables contained in the given partition need to be used for its verification.

Because of the decreased state space of the components, we expect to avoid the state explosion problem while performing model checking, or if it happens, the same decomposition procedure can be applied recursively to the components of interest. After each component has been validated, the resulting abstract model that will contain fewer states should be validated.

\subsection{Decomposition of Variable Dependency Graphs}

One approach to specification decomposition has been presented in [6]. The authors propose an algorithm for slicing system specifications represented with Colored Petri Nets. The slicing is performed by selecting a particular node of interests (CPN place or transition) and following the control flow of the CPN backwards in order to identify arcs, places, guards and transitions that lead to that node. Cukic et al. [4] argue that slicing the specification improves the understanding of the complex system models and helps with identifying high-risk components early in the life cycle.

The underlying ideas are very similar to our current approach: We want to decompose complex system specifications to smaller, more manageable and understandable parts. Instead of ad-hoc decomposition criteria, we propose using minimum coupling, i.e., the boundaries of system components should have minimal coupling (information exchange and/or control connectivity) with the rest of the system. 
Several abstraction and slicing methods for SCR have been proposed in [12][61]. We summarize them below.

\section{Removal of irrelevant variables.}

This method is similar to the "program slicing" technique [40], which removes irrelevant variables for the purpose of program analysis and understanding. Based on the property that we want to verify, only the relevant variables for this particular property obtained by reflexive and transitive closure on the dependency relation are preserved. This method is fully automated and already implemented in the existing SCR toolset.

\section{Removal of detailed monitored variables.}

If a relevant variable for a given property is the only one that depends on a set of monitored variables, then these monitored variables can be removed, and the relevant variable can be treated as a monitored variable.

\section{Replacement of a detailed variable with an abstract variable.}

In this case, the domain of a detailed variable is partitioned in equivalence classes and its size (the number of distinct input values) is reduced to the number of classes taking one representative for each class.

Methods 2 and 3 have not been fully automated [13]. We show how the min-cut graph algorithm can be used to decompose SCR specifications, as well as to automate the abstraction Method 2. Our methodology provides automatic abstraction of irrelevant monitored variables and provides guidance on how to perform verification and validation of system models. The specification and domain experts can focus their verification and validation efforts by combining the results and providing evidence for the correctness of the system as a whole.

In order to apply the min-cut algorithms, we transform the dependency graph produced by the SCR tool to an undirected graph having specific properties of interest. In general, we want to identify internal system variables that represent the points of minimal 
coupling and, consequently, module boundaries. Therefore, partitioning cuts can be applied to the vertices of the dependency graph only, and not to the edges.

We will represent each variable $v$ of the specification by two vertices $v_{\text {in }}$ and $v_{\text {out }}$ connected by the undirected edge $\left(v_{i n}, v_{\text {out }}\right)$. The weight of the newly introduced edge will depend on the type of the variable. We do not want to split monitored (input) and controlled (output) variables, since they cannot imply meaningful system partitions. Therefore, these edges will have infinite weight, $w\left(v_{\text {in }}, v_{\text {out }}\right)=\infty$. Conversely, term and mode variables will have their weights set to $1, w\left(v_{\text {in }}, v_{\text {out }}\right)=1$. Each undirected edge $(v, u)$ of the dependency graph $\left(v_{\text {out }}, u_{\text {in }}\right)$ will assume weight $w\left(v_{\text {out }}, u_{\text {in }}\right)=\infty$, as we do not want to cut any existing dependency edges either.

This transformation is linear on the number of vertices and edges requiring $O(n+m)$ time. It produces an undirected graph with $2 n$ vertices and $m+n$ edges. The choice of the cost of the edges in our transformed graph will force the min-cut algorithm to produce cuts, as intended, on vertices only. Figure 6 presents an example of the dependency graph transformation.

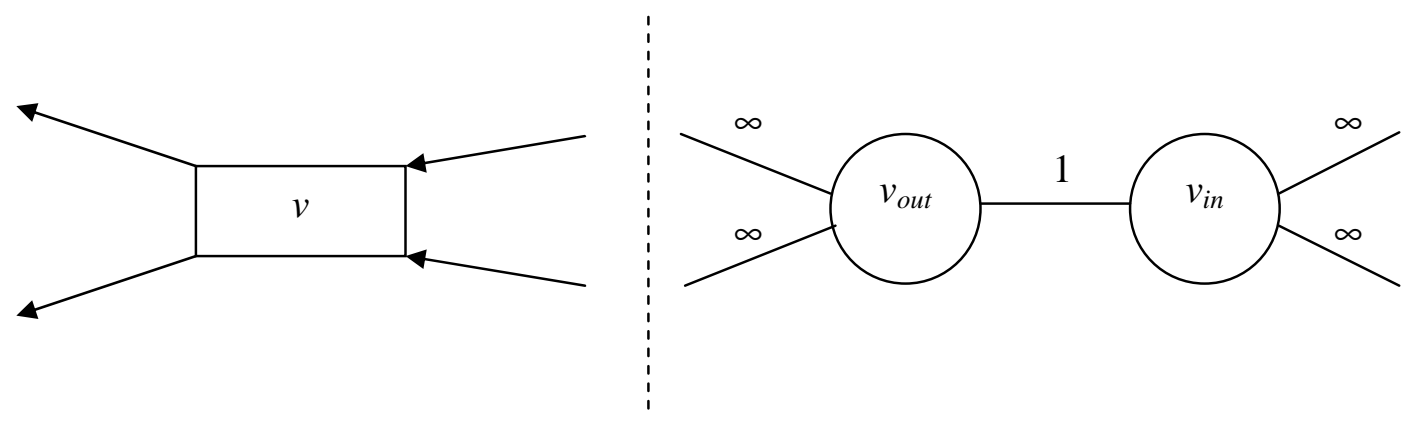

Figure 6: Transformation of the dependency graph to undirected graph.

The application of the min-cut algorithm will find a partitioning of the vertices of the transformed graph in two sets, which minimizes the cost of the edges connecting the both sets. Thus, the cut will contain one or more edges with cost 1 and no edges with infinite cost, thus performing partition of the variables of the specification that crosses the minimal number of variables. The min-cut algorithm can be recursively applied to the 
obtained partitions in order to further decompose the system. Since our transformation is linear and does not expand significantly the size of the problem, the time required to calculate the minimum cut is still polynomial with respect to the size of the specification.

Theorem 4: If an SCR variable $r$ is the only variable which depends on several monitored variables $r_{1}, r_{2}, \ldots, r_{i}$, then a minimum cut of the transformed graph will split this variable.

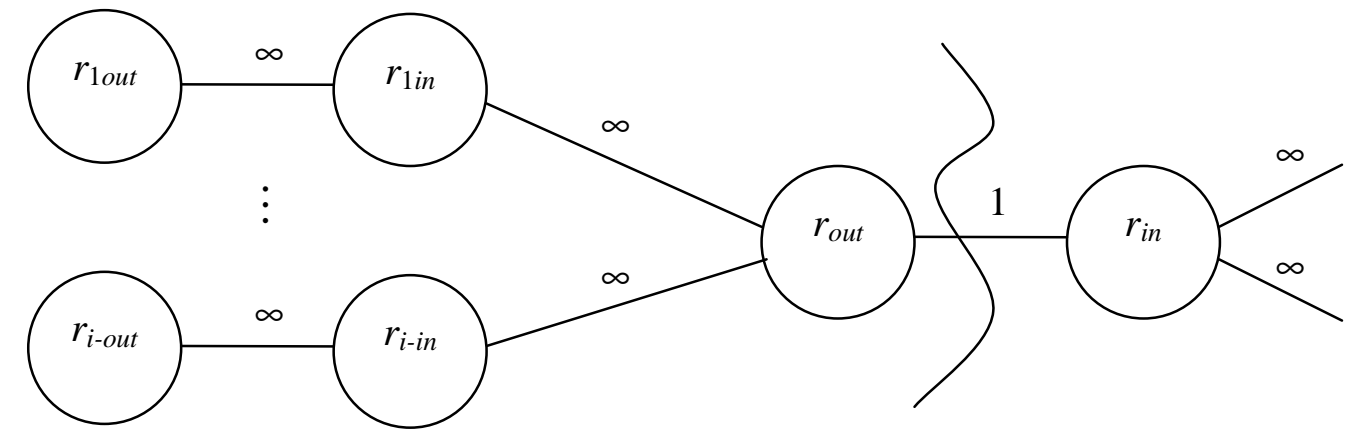

Figure 7: Minimum cut of the transformed graph of a variable directly dependant on several monitored variables.

The proof is intuitive, by construction (see Figure 7). If we generate the transformed graph of this specific dependency, then the cut splitting the variable $r$ has a cost of 1 . This is also a regular cut of the transformed graph because there are no other variables that depend on the given set of monitored variables. Consequently, it is a valid minimum cut, which will be identified by the algorithm.

Corollary 2: The proposed methodology automates the abstraction Method 2 - Removal of Detailed Monitored Variables.

Corollary 2 follows directly from Theorem 4. Calculating the minimal cuts of the transformed graph will automatically identify the variables to which this abstraction method can be applied. 
The proposed methodology not only identifies the variables that directly depend on a set of monitored variables, but it also identifies the variables that are deeper in the dependency graph and have minimal coupling with the other parts of the system. These variables, according to our hypothesis, are the "semi-controlled" variables defining the boundaries of the subsystems contained in the specification. However, we must note that this hypothesis might not hold, i.e., there are many ways one can write an SCR specification for a given system, some of them better than others. Different specifications might not follow generally recognized rules for writing good specifications. There is a list of properties that a specification should have; for example: being complete, unambiguous and minimal. However, from the $\mathrm{V} \& \mathrm{~V}$ perspective, the most desired property and the most difficult to achieve is for the specification to be easily verifiable. We argue that one measure that provides insight if a specification is "open" to verification methodologies is whether it is decomposable, and one such test is our proposed decomposition methodology.

\subsection{The Strategy for Verification of Decomposable Models}

Based on the theory presented in Section 3.3, we propose the following strategy for verification of decomposable models. As stated previously, the overarching idea is to apply a divide and conquer approach in the verification of SCR specifications. The mincuts decomposition results in partitioning of the variables, where each partition represents a smaller component of the complete specification. The verification of these parts is performed against the properties involving only variables contained in the given partition.

Jeffords and Heitmeyer in [59] present a strategy for verification that performs abstraction by removing a single variable from the specification and uses the automatically generated invariants [60] for the abstracted variable to strengthen the deduction rules. They state that the problem on how to decompose a given system for performing compositional verification is still open, and should be automatable and accessible to non-experts. Our approach extends their work by considering components of the specification (i.e. sets of variables) which have low coupling and information 
exchange with the rest of the specification. Instead of automatically generating invariants for the abstracted components, our approach is to specify properties at the component level and later try to prove that they are invariants and use them in the certification process of the system as a whole.

Given a specification of a system $\Sigma=(V, S, \Theta, \rho)$ and a set $P$ of desired properties to be proven as invariants, we apply the following steps.

Step 1. Apply the decomposition algorithm to obtain partitioning of the set of variables $V$, to disjoint subsets $V_{1}, V_{2}, \ldots, V_{n}$.

According to our heuristic hypothesis, each of these sets should represent one component of the system.

Step 2. Construct each component system $\Sigma_{i}$ from $V_{i}, i=1$ to $n$, and $\Sigma$ as follows:

(a) Delete from $\Theta$ the initial state definitions of variables in $V-V_{i}$, and assign the result to $\Theta_{i}$.

(b) Delete from $\rho$ the functions defining the variables in $V-V_{i}$, and assign the result to $\rho_{i}$.

This step essentially makes all variables in $V-V_{i}$ to behave as monitored variables for the subsystem $\Sigma_{i}$. In other words, by removing the functions defining them, these variables can assume any allowed value non-deterministically. This introduces incompleteness in the verification process in the following sense: in order to prove some properties for the component that are necessary for the system correctness, we might need to rely on some explicit or implicit assumptions that this component makes about its environment or other interfacing components. The goal is to make all needed assumptions explicit during the specification process, i.e. the created verification checklist of properties should be complete in order to allow verification of the system. 
Step 3. For each component $\Sigma_{i}$ select those properties from the set $\mathrm{P}$ that depend only on the variables found in $V_{i}$. Try to prove that these properties are invariants for the component and, consequently, from the corollary of Theorem 3, invariants for the system.

Our minimum cut decomposition approach guarantees that the component $\Sigma_{i}$ has the least number of dependencies on other system variables. Consequently, this selection of properties ensures that most of the variables $V-V_{i}$ will be abstracted away by using the abstraction method 1 and reduces the incompleteness of the component verification process.

Step 4. Use the compositional verification rules together with the invariants proven in the previous step to prove the rest of the properties, or those properties which have failed in step 3 .

Each derived component represents an abstraction of the complete system. However, components usually allow auxiliary behaviors that might invalidate the properties we would like to prove. The min-cut decomposition approach minimizes the dependency of a single component on external variables, thus reducing auxiliary behaviors and making it often possible to prove invariants for the system without using extra invariants.

Step 5. The properties that depend on variables contained in several components can be demonstrated in parts if they can be represented as conjunction of component properties. Or, they can be proven by creating larger subsystems from unions of the required sets of variables $V_{i} \cup V_{j}$ and repeating the steps 2, 3 and 4 .

Assuming that a given system specification $\Sigma$ is decomposable; the proposed strategy should improve the time and memory requirements for verification of the system properties. In Chapter 7 we demonstrate how an SCR specification, which originally was not decomposable, can be refactored into a decomposable specification. We identify the main principles for designing decomposable specifications and use the proposed strategy to verify the required system properties. 


\subsection{Combining Verification and Testing results}

Because of the scalability problems with formal methods and the common use of testing, combining both methodologies has been an obvious research goal. The main argument behind this combination implies that software reliability can be increased by applying different analysis methods. Sharygina and Peled [49] propose a combined methodology of testing the checked model against the actual code, as part of applying formal methods (Figure 8). More information on the subject of can be found in Peled's book [19].

Another approach for combining testing with formal models is for the purposes of automatic generation of tests [5][42]. Based on coverage measures of the formal specification model, model checking is used in order automatically to generate tests suites. These test suites are later used on the actual code. Many researchers have investigated models for reliability estimation based on code coverage. It would be interesting to add the specification coverage into the picture, and see what the correlation is.

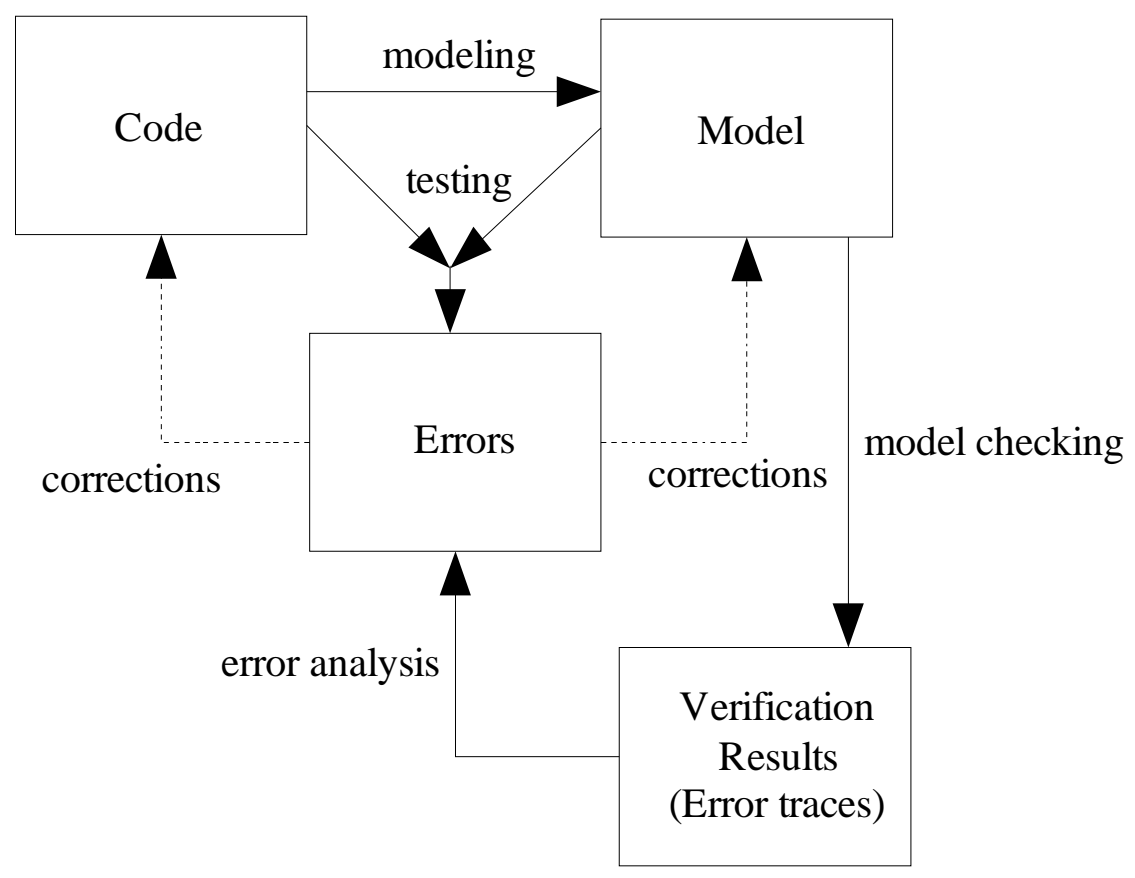

Figure 8: Combining Testing and Model Checking. 
Partition software testing is also a promising area to introduce formal specification models. There is plenty of research being done on comparing randomized testing with partition testing, pointing out when it is more beneficial to use one or the other [52]. However, currently domain partitioning is largely an informal process. Specification models can be used to derive the domain partitioning and test generation based on obtained equivalence classes.

Our interest is mainly on the validation of the formalization phase, i.e., whether the formal specification and its model are correct with respect to the informally captured requirements. If a correct formal model has been obtained, we can use other techniques like rewriting (program synthesis) to obtain correct executable code [26].

\subsubsection{Standard component based Reliability Estimation}

In the SCR model, a single update step sequentially executes the function definitions of the specification modifying the affected variables accordingly. For example, in Figure 9 we see the source code of the update step of the SIS specification that is automatically generated from the code.

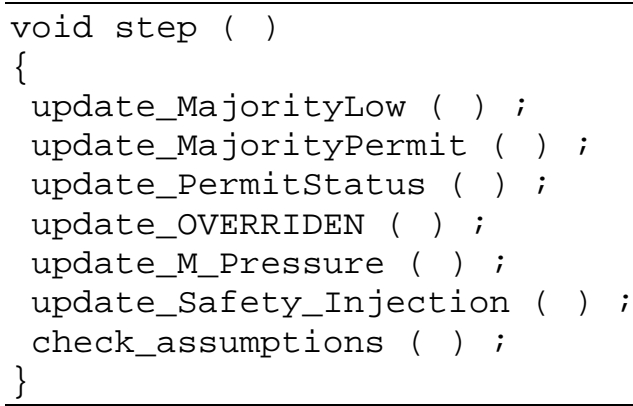

Figure 9: Executable $\mathrm{C}$ code for the SIS specification.

According to standard reliability models, the reliability of the single step of execution will be the product of the estimated reliabilities of each of the functions, or in the SCR case, the tables specifying the variables $v_{i}, i=1 . . n$. The assumptions are that component 
failures are independent and that there is no error propagation between the components [51].

$$
R(S)=R\left(v_{1}\right) \cdot R\left(v_{2}\right) \cdot R\left(v_{3}\right) \cdot R\left(v_{4}\right) \cdot \ldots \cdot R\left(v_{n}\right)
$$

Our decomposition algorithm generates partitions of these variables, which we consider to be components. Consequently, we can rearrange and rewrite the reliability equation combining the parts representing a single component, and present the system reliability as the reliability of components.

$$
R(S)=R\left(c_{1}\right) \cdot R\left(c_{2}\right) \cdot R\left(c_{3}\right) \cdot \ldots \cdot R\left(c_{k}\right)
$$

In case a component is formally verified, i.e., all corresponding properties have been demonstrated to hold, it is usually assumed that its reliability is $R\left(c_{i}\right)=1$. If the formal verification is out of reach, we can apply standard reliability growth models based on testing to estimate the component reliabilities. As we have stated previously, reliability growth models cannot be applied to high assurance systems, however they still can be used for systems or components that are not highly critical.

These approaches are rather simplistic and based on assumptions that are difficult to justify and rarely hold in practice. We propose a different correctness estimation approach, which is based on the stated properties about the components and the system.

\subsubsection{Property-based Correctness Estimation}

The list of stated properties represents our "check-list," which must be demonstrated in order to certify the correctness of the system. This list must be complete and take into account all required aspects of the system operation. Ideally, we would like to be able to formally verify and demonstrate the correctness of each of these properties and consequently the correctness of the system. However, we are faced often with scalability problems in applying formal methods, and we must rely on standard quality assurance techniques like testing or manual inspection. Once in the realm of informal techniques, usually we cannot be $100 \%$ sure of the validation outcome and think in the terms of 
probabilities of correct execution of the system, system failure rates, or reliabilities. Thus, the probability that our system is correct is equal to the probability that all stated properties are correct. Since we are dealing with high assurance systems, no observed failures of the properties are tolerated. If a property is observed as failed during testing or verification, then the system is considered as not being correct.

The properties can be dependent on each other, i.e. we do not assume that they are all independent. The dependency emerges from the assumptions that some of the component make about their environment that are needed in order for them to operate correctly and establish their invariants. These assumptions must be stated as properties and eventually proven for the respective interfacing components, i.e. the list of the properties must be complete, allowing verification of all stated properties. The independent properties would be the first ones that will be proven by applying our verification strategy. They are used in the next iteration in order to prove other properties that might depend on them, and we repeat this process until all properties have been proven as invariants for the system.

When performing verification of the complete system by using model checking, we are trying to establish the correctness of the formula:

$$
\mathrm{S}=\alpha_{1} \wedge \alpha_{2} \wedge \alpha_{3} \wedge \ldots \wedge \alpha_{\mathrm{n}}(\text { Eq. } 12)
$$

where $\alpha_{i}$ are the stated system properties. If our verification task is unsuccessful, we should try to estimate the probability of this formula being true. For systems that are not highly critical this is usually done by performing testing of the complete system, looking for failures of the stated properties and using reliability growth models.

The problem on how to quantify this probability for high assurance systems or when there are no failures observed remains open. One suggestion might be to use the fraction of the explored states and the total states of the system, or try to incorporate other coverage measures like the structural coverage (to be presented in Chapter 5). Since the testing or verification in this case is performed on the complete system, we do not need to 
make the assumptions of the components explicit - they are incorporated in the system model.

$$
P_{\Sigma}(S)=P_{\Sigma}\left(\alpha_{1} \wedge \alpha_{2} \wedge \alpha_{3} \wedge \ldots \wedge \alpha_{n}\right)=P_{\Sigma}\left(\alpha_{1}\right) \cdot P_{\Sigma}\left(\alpha_{2}\right) \cdot P_{\Sigma}\left(\alpha_{3}\right) \cdot \ldots \cdot P_{\Sigma}\left(\alpha_{n}\right) .(\text { Eq.13) }
$$

When dealing with large systems, we are faced with the limits of the state exploration methodologies and rarely can verify the properties on the complete system. Our component-based verification strategy breaks up the verification task on components. The advocated approach is that each component should state the properties that are guaranteed based on assumptions about the environment and the other components it depends on.

Due to the performed abstractions, establishing the correctness of a property might be dependent on other properties. Therefore, during the application of our verification strategy, we try first to demonstrate the correctness of properties that are independent on other properties, and later use them as assumptions in the verification process. In general, assuming the properties are ordered according to their dependencies and there is a single property per component, the verification process can be decomposed as following:

$$
\begin{aligned}
& P_{\Sigma}(S)=P_{\Sigma}\left(\alpha_{1} \wedge \alpha_{2} \wedge \alpha_{3} \wedge \ldots \wedge \alpha_{n}\right) \geq P_{\Sigma 1}\left(\alpha_{1}\right) \cdot P_{\Sigma-\Sigma 1}\left(\alpha_{2} \wedge \alpha_{3} \wedge \ldots \wedge \alpha_{n} \mid \alpha_{1}\right) \\
& \geq P_{\Sigma 1}\left(\alpha_{1}\right) \cdot P_{\Sigma 2}\left(\alpha_{2} \mid \alpha_{1}\right) \cdot P_{\Sigma-\Sigma 1-\Sigma 2}\left(\alpha_{3} \wedge \ldots \wedge \alpha_{n} \mid \alpha_{1} \wedge \alpha_{2}\right) \\
& \quad \geq P_{\Sigma 1}\left(\alpha_{1}\right) \cdot P_{\Sigma 2}\left(\alpha_{2} \mid \alpha_{1}\right) \cdot P_{\Sigma 3}\left(\alpha_{3} \mid \alpha_{1} \wedge \alpha_{2}\right) \cdot \ldots \cdot P_{\Sigma n}\left(\alpha_{n} \mid \alpha_{1} \wedge \alpha_{2} \wedge \alpha_{3} \wedge \ldots \wedge \alpha_{n-1}\right)
\end{aligned}
$$

The dependencies between the properties, or at least between the components on which the properties are stated, are evident on the dependency graphs of the SCR specification under analysis. In general, each component implicitly depends on properties established for components that precede it in the dependency graph. This formula is similar to the standard component reliability estimate formula, but it stresses that correctness of components must be evaluated in the context of their application. Recent work in such component-based reliability frameworks for the special case when components have no internal states is presented by Hamlet et al. [18]. 
Consequently, if the proof of a property is out of reach, we must estimate the probability of correctness using either the complete system as in (Eq. 13) or component-based evaluation as given in (Eq. 14).

\subsection{Example Application of Decomposition}

We demonstrate our methodology of component-based verification and combining testing and formal analysis by applying it to the described Safety Injection System specification.

The dependency between the variables in an SCR specification can be visualized by using the Dependency Graph Browser tool of the SCR toolset. Figure 10 presents the dependency graph for the Safety Injection specification.

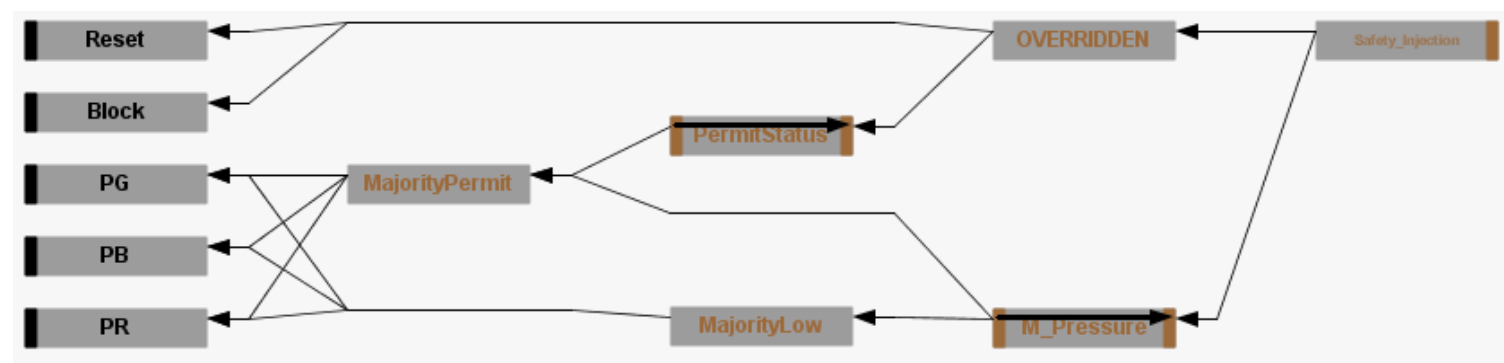

Figure 10: Dependency Graph of the Safety Injection SCR specification.

The SCR model keeps track of two values for each variable at any distinct point of time the current value, and the next state, or the "new" value. A change in one of the monitored variables, e.g., setting the next state value of the Block variable to true while the current value is false, will cause update in the variables that depend upon new value of Block. However, dependencies can also exist on the previous value of a specific variable, so the complete dependency graph contains both types of dependencies (dependency on the new values, and dependency on the old values). If we select to draw the dependency only on the new values, we would get the same graph as in Figure 10, except that the arrows connecting M_Pressure and PermitStatus to themselves would not be there. Figure 11 presents the dependency graph for the old values of the variables. 


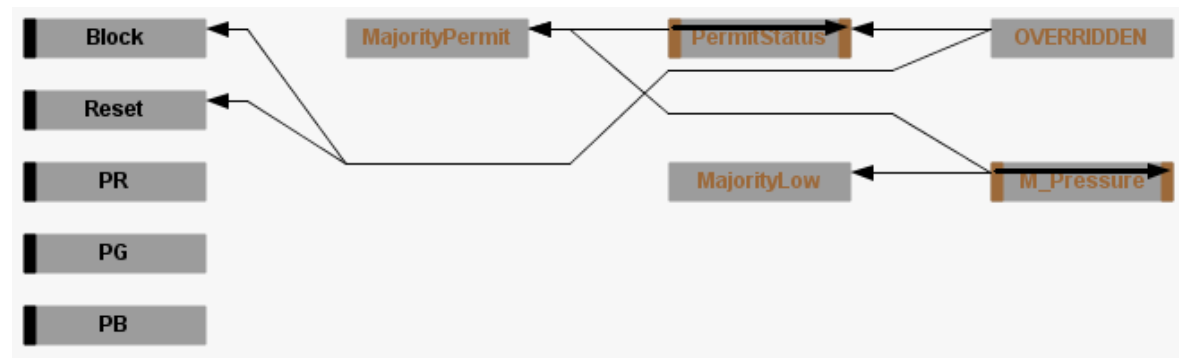

Figure 11: Dependency of the variables on the "old" values.

Performing the decomposition on different types of graphs can provide additional insight on the cohesion between the components and the types of dependency between them whether they depend on the "new" or "old" value of some component.

We implemented our decomposition methodology using a combination of JAVA and C implementations of the minimum cut algorithms [76]. From a given SCR specification, we extract the dependency graph information using the SCR toolset, and then use our tool to perform the decomposition. Currently, the prototype tool just reads the extracted dependency information and outputs the names of the variables that belong to different min-cut partitions of the dependency graph. Further analysis and application of our verification strategy is performed manually using the SCR toolset. In future, we plan to incorporate the decomposition algorithms within the SCR toolset, thus automating the application of our verification strategy. In addition, we noticed the need to allow the user to assign different weights to the dependency graph edges, guiding the min-cut partitioning as mentioned in Section 7.2.

\subsubsection{Decomposition of the specification}

The results of applying our decomposition algorithm on the complete dependency graph "SISall.dg" are given in Figure 12. The main module responsible for pressure monitoring and the incorporated majority voting has been identified, and we obtain the same decomposition as presented in Figure 5. Often the dependency on the old variables is what makes the dependency graph more complex and limits the identification of 
components by our algorithm. This can be resolved in several ways, either by possibly relaxing the costs of cuts on some of the dependency links (e.g. the "old" dependencies), or by running the decomposition algorithm on both dependency graphs as we decided to do in this example. Therefore, we perform additional run on the dependency information for the new values "SISnew.dg" (Figure 13).

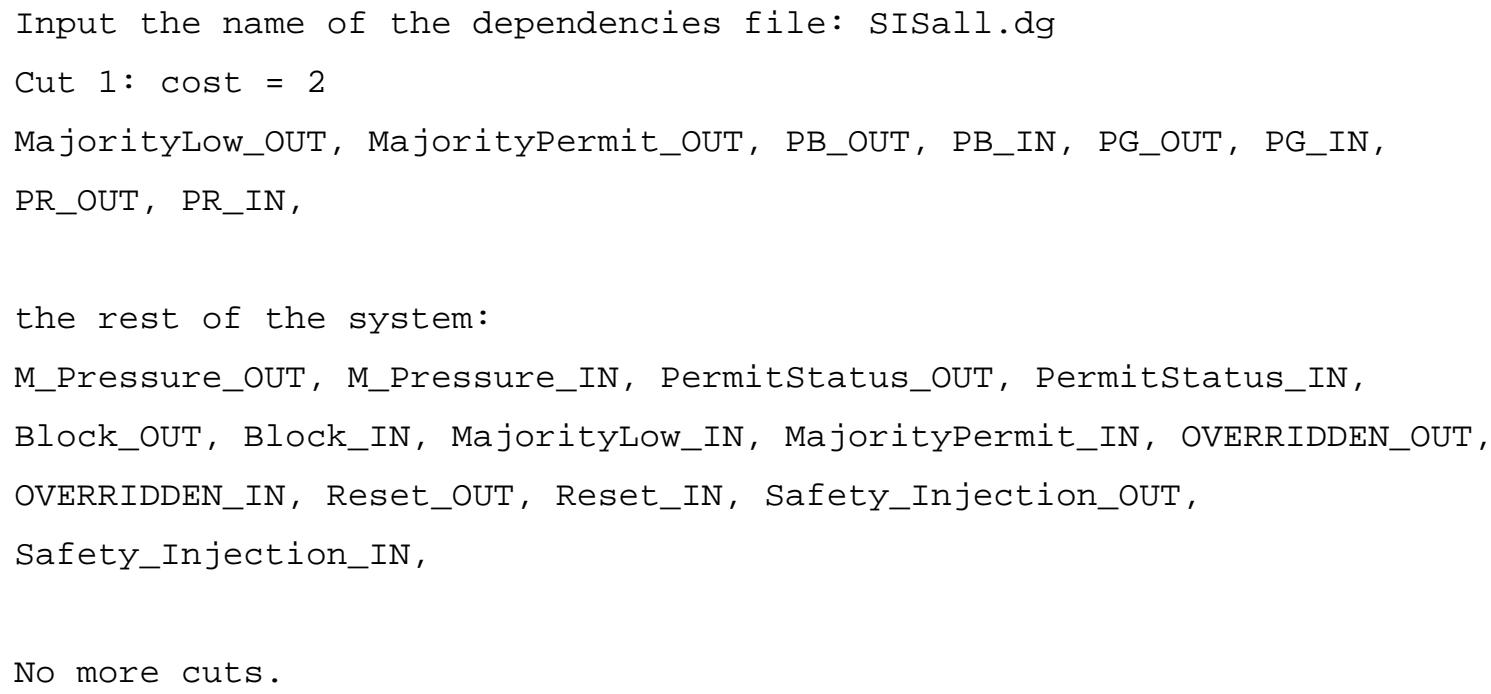

Figure 12: Decomposition of the complete dependency graph of the SIS specification.

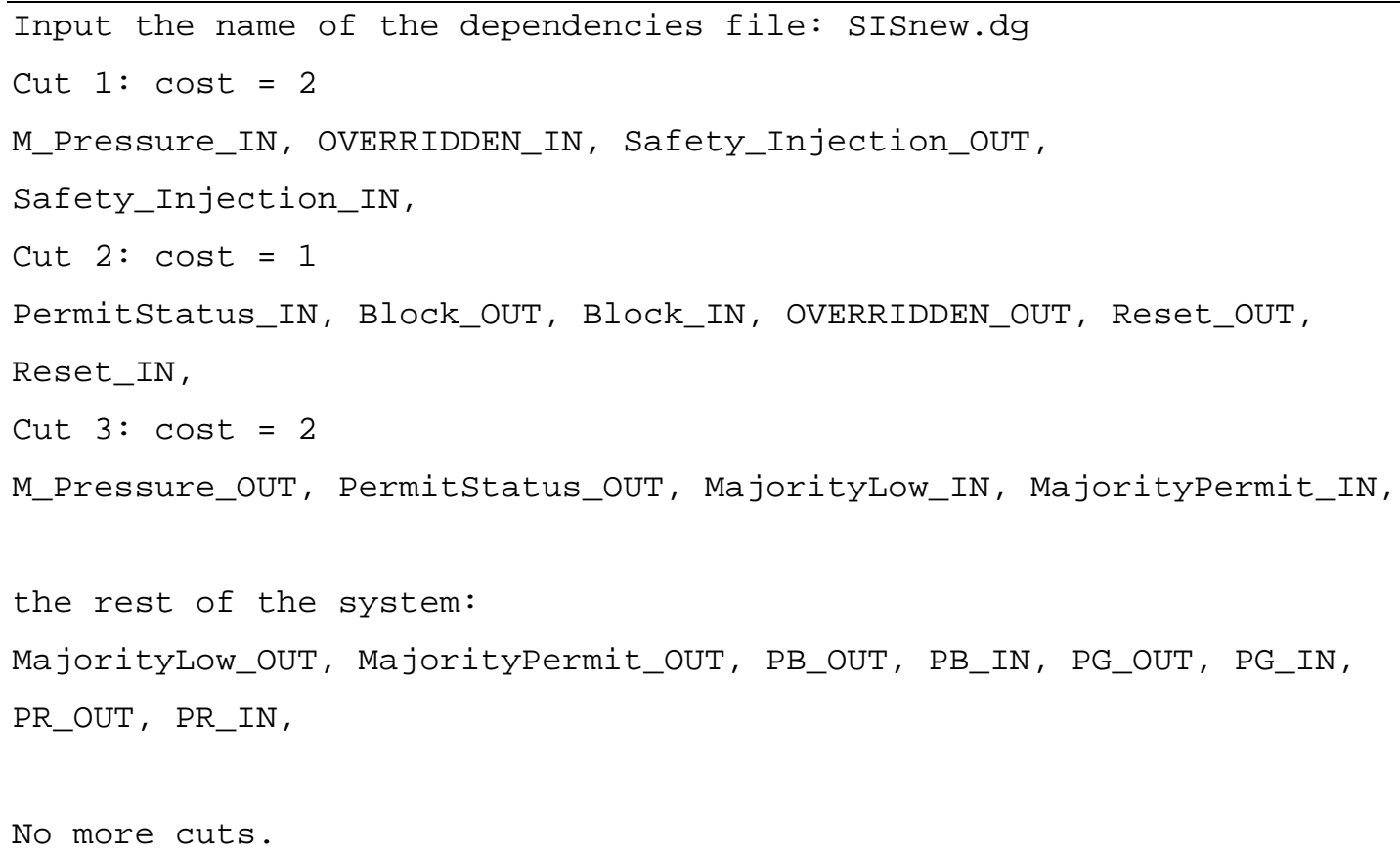

Figure 13: Decomposition of the "new" dependency graph of the SIS specification. 
In addition to the above-identified component, the rest of the system was decomposed in 3 modules (Figure 14). Having the decomposed specification, we can proceed with the validation and verification tasks.

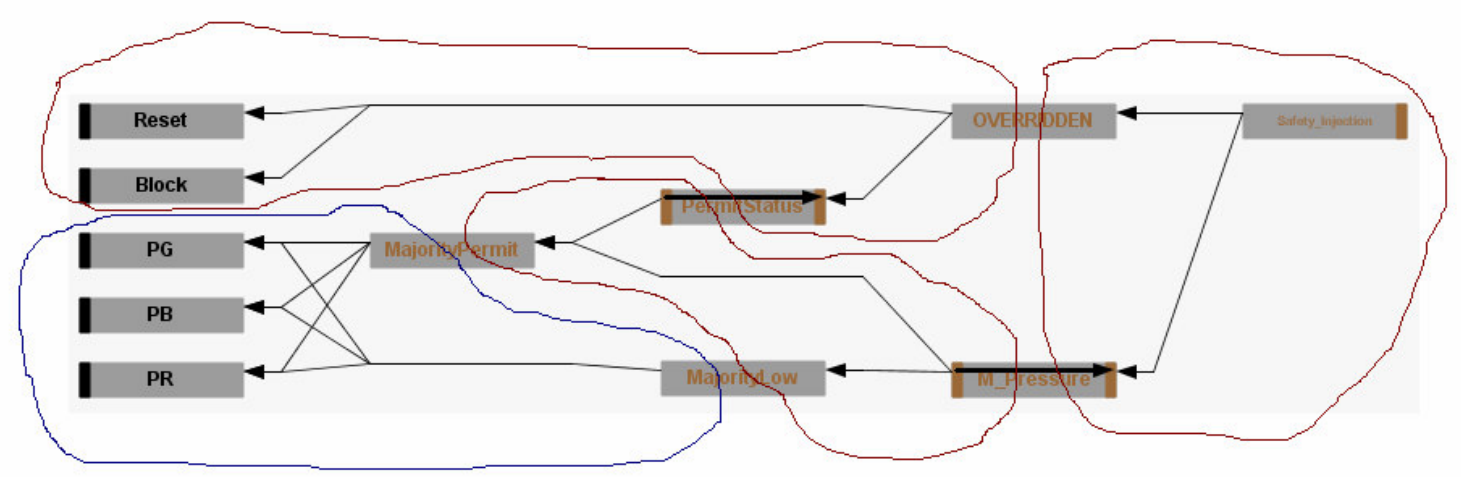

Figure 14: Decomposed Safety Injection SCR specification.

\subsubsection{Verification of the Properties}

The assumption of our methodology is that we have defined the complete list of properties that provide assurance in the correctness of the given specification. For this example, we will use the following properties:

Voting: $(P G>=$ permit $) A N D(P R>=$ permit $)=>$ NOT (MajorityPermit OR MajorityLow)

OverrideWorks: Reset $=O N=>$ not $O V E R R I D D E N$

InjectionOff: $(P G>=$ permit $) A N D(P R>=$ permit $)=>($ Safety_Injection $=$ OFF $)$

Although this list is far from being complete, it is useful for the demonstration of the methodology.

As we mentioned in Section 3.2.2, the problem we encounter when performing model checking of the complete specification with SPIN is the large state space of the voting 
component. Thus, after identification of the components by our decomposition algorithm, we will first try to prove the properties that are stated on component boundaries.

The property Voting includes only variables from the voting component and this component does not depend on any other components. Unfortunately, since the input domain of this component is very large, SPIN is unable to demonstrate the correctness of this property using the available memory, but also does not find violations.

OverrideWorks includes only variables from control component identified by the first decomposition. Figure 15 presents the results of model checking the control component for this property, demonstrating that it holds for the reduced system. We performed the full state-space search. It took only a few seconds using 35MB of memory. Consequently, this property also holds for the complete system.

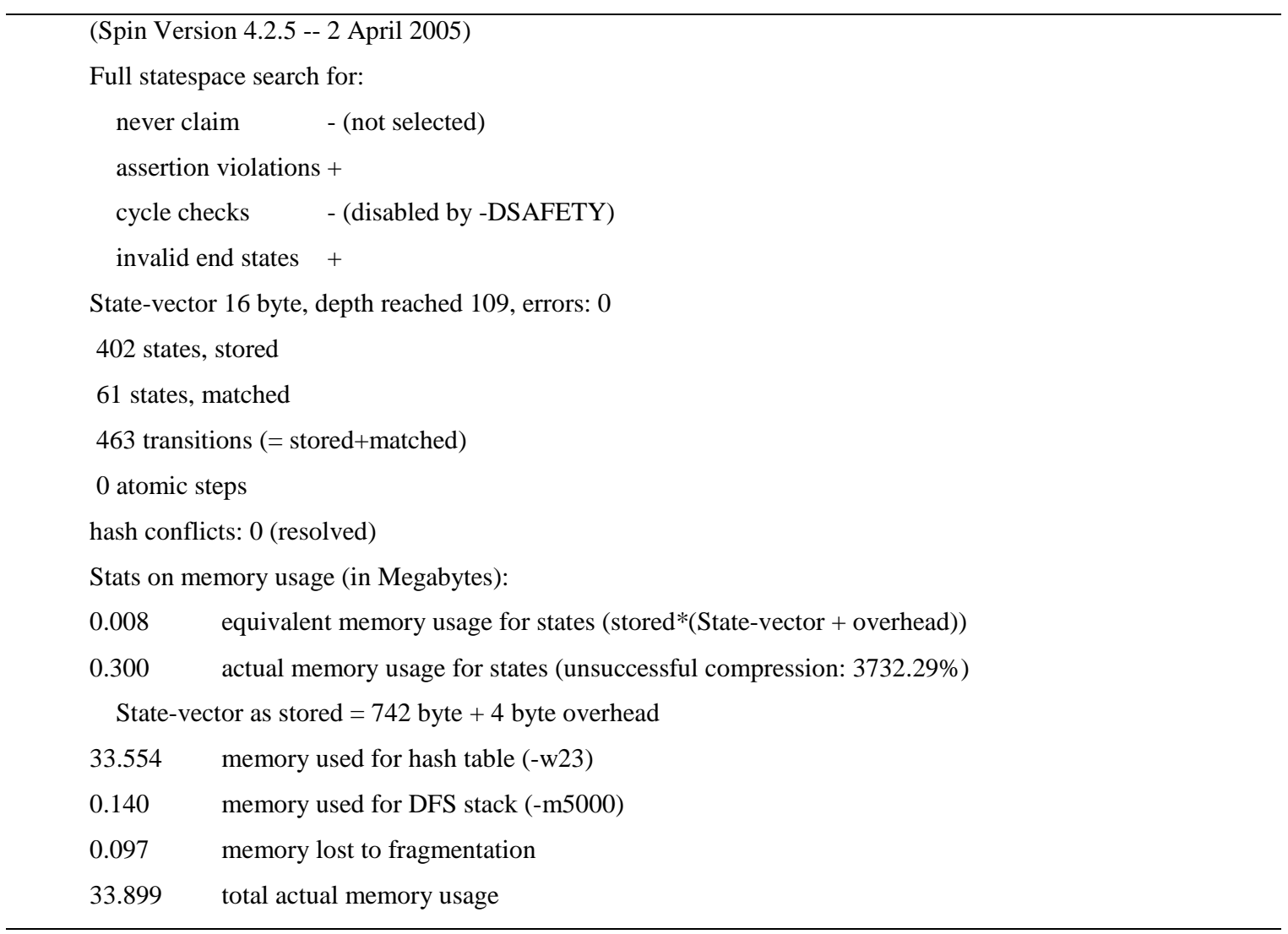

Figure 15: Model checking the control component. 
The InjectionOff property includes variables from both components, and as we know model checking the complete system with SPIN will be infeasible. Therefore, we use deduction rules to try to prove if this property holds for the control component, assuming that the Voting property is true. We try to present this property as a conjunction, as suggested in Step 5 of our verification strategy.

InjectionOff $=$ Voting $\wedge$ NOT $($ MajorityPermit OR MajorityLow $)=>$ Safety_Injection $=$ OFF

The second part of the conjunction now includes only variables from the control component, and is successfully verified by SPIN. Consequently, we can conclude that if the Voting property holds, the complete checklist can be verified and we can certify the correctness of the system. By using other tools like SMV and Salsa we were able to prove the Voting property, demonstrating the effectiveness of symbolic model checking and inductive theorem proving tools vs. state based model checking in this particular case.

If we were unable to establish the correctness or the failure of the Voting property, we would need to give an estimate of our belief that it holds true. The most obvious approach is to calculate the fraction of the explored states and the total states of the corresponding component. Thus, the estimate of the correctness of the system in this case according to our methodology would be:

$$
\begin{aligned}
& P(S) \geq P_{\text {voter }}(\text { Voting }) \cdot P_{\text {control }}(\text { SafetyOff } \mid \text { Voting }) \cdot P_{\text {control }}(\text { OverrideWorks })= \\
& =P_{\text {voter }}(\text { Voting }) \cdot 1 \cdot 1=\frac{\text { Voter }: \text { explored states }}{\text { Voter }: \text { reachable states }} \geq \frac{\text { Voter }: \text { explored states }}{\text { Voter }: \text { total states }} .
\end{aligned}
$$

\subsubsection{Testing the specification}

The properties that have not been proven correct must be evaluated either by testing or by inspection. In Chapters 5 and 6, we describe the approaches used for testing and validation of SCR specifications. We evaluate the effectiveness of random testing approaches for early fault detection and investigate the achieved structural coverage measures. We also advocate the use of visual environments for manual testing purposes. 
Using these visual environments, the domain expert can execute scenarios of the system operation and observe the system responses visually. The tools track the coverage of the executed tests, and store the scenarios for recording and certification purposes (e.g. the same scenarios can be re-executed if the specification changes, observing the differences in the induced coverage between both versions). For example, in Figure 24 we see that during the testing, MajorityPermit and MajorityLow variables have reached $100 \%$ coverage, increasing our confidence that their specifications are correct and providing evidence of the correctness of the Voting property.

These testing coverage metrics are defined on structural artifacts of the given specifications (e.g. events or conditions), and are different from the state-space coverage. Achieving complete (100\%) state-space coverage ensures the correctness of the property under verification, while complete structural test coverage is not sufficient to make this claim. It just provides further evidence of the correctness of the specification. 


\section{Chapter 5}

\section{Testing and Coverage of Formal Models}

Software systems pose significant challenges for quality assessment. This is mainly caused by the prohibitive complexity of many practical software applications. Despite the enthusiasm in the research community about the development of formal methods for software verification and validation, they are rarely used in industry, due, in part, to scalability problems. Many current software applications are outside of the reach of formal verification approaches and tools. Consequently, testing is the dominant methodology currently used in industry to assess the quality of software.

Software testing has another set of limitations. It is incomplete, so not encountering a failure during the testing phase does not mean that the software is fault-free. If performed manually, it leads to increased costs of the software development process since it is labor intensive and depends on the specialized skills of the testers to find bugs in the code. Thus, the tendency is to automate the testing process, either by creating automated test case replay tools, or by randomly generating input sequences and using an oracle to validate the correctness of the output.

In the case of the replay tools, the burden is moved to creation of the test case database and its maintenance as the system evolves. Random Testing [56] on the other hand can generate a large number of input cases automatically; however, the development of the 
oracle that would distinguish the failures during test execution is usually not an easy task. If the oracle produces too many false positives needing human review and resolution, it would significantly increase the cost, while false negatives would result in undetected errors in the code.

One approach to the oracle problem, especially in the domain of high assurance systems, is to use software requirements specifications written in some formal notation as an oracle [14][63]. The main assumption in this methodology is that the formal model of the system representing the requirements specification is "correct." Having a correct formal model can be very beneficial in the software development process since it can be used for automated generation of tests based on defined coverage metrics on the model [5], or it can be used for automated generation of source code which might represent an initial implementation of the system [26]. In our research, we are focusing on methodologies that can be used to verify such formal software models.

\subsection{Random Testing of Formal Models}

Our motivation is based on observation that incomplete random testing tools can be used most effectively early in the modeling process, e.g., while writing the SCR requirements specification for a given system. In this phase, we are still not sure if we have built the correct model or have correctly identified and stated the properties that the model must satisfy. Therefore, it is useful to have a way to quickly identify errors in the operational model or in the properties themselves, and make appropriate corrections early in the lifecycle.

As we get closer to a final version of the SCR requirements specification, having corrected the errors detected by testing, we can then apply complete verification tools like SPIN, if feasible, to demonstrate the consistency between the operational model and the stated properties. 
We use a prototype simulation tool for random testing and debugging of models of finitestate concurrent systems called Lurch [17]. Although Lurch's exploration of the state space is not exhaustive, it can be used to detect errors in large systems quickly using much less memory than any complete-search alternatives. Lurch uses a Monte Carlo, not Las Vegas, random search algorithm [57] in contrast to, for example, a random depthfirst search, in which all nodes are explored but the order is random. Where random search has been used recently in model checking by others, e.g. [3], it has been primarily the Las Vegas approach; this is fundamentally different from our approach as it requires the record keeping of conventional deterministic model checking (the search must keep track of states previously visited) and therefore can not scale to models as large.

To do cycle detection, Lurch stores a hash value for each unique global state in the current path. This requires some additional time and memory and can be turned off if the user is not interested in looking for cycle-based faults. Using the hash value storage needed for cycle detection, Lurch implements an early stopping mechanism that works in the following way: for each path generated, we save hash values for all unique global states visited (this is done already for cycle detection) and compare the number of collisions to the number of new values. When the percentage of new values drops below a user-defined saturation threshold (default is $0.01 \%$ ), the search is terminated. In our experiments, saturation is usually achieved quickly. In addition, when Lurch is allowed to run to saturation, it nearly always produces consistent results identifying the same errors in different test runs of the given model.

The hash values can be also used to determine the number of explored states of the model, providing a way to estimate the probability that a given property holds for the system under analysis. We use this measure as discussed in Section 4.3.2. 


\subsection{Structural Test Coverage of SCR specifications}

During testing or simulation of an SCR specification, we can monitor the structural coverage achieved by the test. These coverage measures represent the extent to which particular parts of the specification have been exercised during testing and increase our confidence in the correctness of the specification. Parts that have good coverage tend to have fewer faults, since the probability of detecting them during the testing phase is greater than in the parts that have achieved lower coverage. However, the exact relationship between code reliability and coverage measures is still an open problem.

There are two types of coverage that can be defined on underlying constructs of SCR specifications, similar to ones defined in [5] or [42], which are primarily used for automated generation of test cases using the specification as "correct" oracle. The first one is variable domain coverage, which tracks if all possible values of a specific variable's domain have been assigned to it by the test cases. Tacking the variable domain coverage is only feasible for domains with small bounds, like enumerated types or booleans. The second one is branch coverage, which tracks if all possible branches defined by events or conditions in the SCR tables have been executed.

Table 10: tNumCReads event table.

\begin{tabular}{|c|c|c|}
\hline Modes for mcStatus & Events & \\
\hline CheckCard & @T(mCardValid) OR @C(mReset) & $@ \mathrm{~F}(\mathrm{mCardValid})$ \\
\hline Error & @C(mOverride) OR @C(mReset) & NEVER \\
\hline ReEnterCard & $@ \mathrm{C}(\mathrm{mReset})$ & NEVER \\
\hline tNumCReads' = & 0 & tNumCReads +1 \\
\hline
\end{tabular}

Table 10 presents the event table for the tNumCReads term variable of the PACS system described in Section 7.1. This variable counts the number of unsuccessful card reads for the current user trying to obtain access through the system. Depending on the current value of the mcStatus variable and the events described in the cells of the table, tNumCReads is either set to zero or increased by one. The keyword NEVER denotes an event that can never become true. 
The branch coverage measures how many times each event in the cells was evaluated to $T R U E$ and to FALSE when the corresponding guard condition was satisfied. For example, for the cell in the first row and second column of Table 10 we count how many times the event @F(mCardValid) is evaluated to TRUE or to FALSE when the mcStatus variable is equal to CheckCard during the execution of the test. If there is no guard condition (i.e. for modeless tables), we consider that the guard is always satisfied. This coverage measure can be further extended to include more than just TRUE and FALSE cases by creating different sets of variables used in the conditions following the ideas of [5] and [24].

The other coverage measure, the variable domain coverage, tracks if all possible values of a specific variable domain have been assigned to it by test cases. The SCR tool itself warns the specifier if some of the values for a given enumerated variable are not used in the specification. With this coverage, we measure how many of them were used by a given test case. If an SCR table contains all the possible assignment values for the variable, then it is easy to see that complete TRUE transition coverage implies complete variable domain coverage, because each of the value assignments must have occurred during the test run. However, for variables with larger domains (e.g. integers like tNumCReads in Table 10) it is often impractical to track this coverage, so we mostly focused on the branch coverage.

It is interesting to compare the defined specification branch coverage to the source code branch coverage. Both, the generated java source code by the SCR toolset, and the automatically generated $\mathrm{C}$ code follow the following structure.

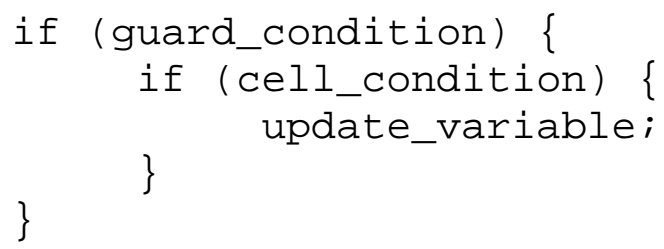

Thus, the observed specification branch coverage closely matches the source code branch coverage within the functions defining the variables of the specification. We expect that any test cases that are executed on the specification, or generated from it, will achieve the same or comparable branch coverage measures on the generated source code. 


\subsection{Empirical Evaluation of Random Testing}

In order to evaluate the efficacy of our random testing approach, we performed a mutation testing experiment. We created 50 error-seeded versions of the PACS specification by hand (PACS is described in Section 7.1, and the original SCR specification is given in Appendix A.1.). Each error-seeded specification contained one error not detectable by automatic checking features of the SCR Tool. For example, since syntax errors or errors involving circular dependencies are detectable by the SCR Tool, we did not use these for seeding. The mutation operations used were:

- Change of operators;

- Change of integer and boolean values;

- Change of variable names.

We believe that these are typical human errors which occur in regular SCR specifications, and should not skew our mutation testing results [30].

We used translation tools to create SPIN and Lurch versions of each error-seeded specification. All error-seeded SCR specifications resulted in syntactically correct SPIN and Lurch input models. We then ran SPIN and Lurch on the 50 error-seeded specifications.

To give an idea of the scale of the experiments, for a verification run of the Promela model generated from the original correct SCR specification, SPIN explored 180 million states. Without compression, the verification would have required $6.8 \mathrm{~GB}$ of memory. In order to perform a full verification run, we used the minimized automaton compression option, set for a state vector of 44 bytes, and had to increase the depth limit for the search to 3.2 million steps (default is 10,000). This verification run took about 30 minutes on a $2.5 \mathrm{GHz}$ desktop machine with $512 \mathrm{MB}$ of memory. 


\subsubsection{Error Detection}

SPIN with the settings appropriate to perform the complete verification, detected property violations in 43 of the 50 specifications. We assume that for the remaining 7 specifications the seeded errors did not cause violations of any specified properties and consider that they are equivalent to the original correct specification. All defined properties for the PACS specification were simple safety properties, represented as assertions in the SPIN and Lurch models.

For 35 of the 50 error-seeded specifications, Lurch detected a property violation. Because of Lurch's incomplete random search, it is possible to obtain different results in different runs. So, we ran Lurch 10 times on each specification, with default options (including the "saturation"” stopping criterion described previously). The maximum time for any Lurch run was about 15 seconds. For all but two of the specifications, Lurch found violations in either all or none of the runs. We counted Lurch as detecting violations only if they were found in all ten runs.

Consequently, the random testing approach in Lurch was successful in identifying $81.39 \%$ of the mutants requiring a small fraction of the time needed to perform the complete verification by using SPIN.

\subsubsection{Induced Coverage}

As a second part of the experiment we wanted to take a closer look at the induced structural coverage of the performed tests on the PACS SCR model.

The input sequences generated by the Lurch tool were exercised by instrumented simulators of the SCR models tracking the previously defined coverage measures. Table 11 gives the cumulative coverage numbers for portion of the variable definitions from a single Lurch run on the correct specification. Some of the variables have more than 6 cells, and we have truncated the table because of space constraints. In this table we can find the defined branch coverage of all 6 cells of the Table 10 specifying the tNumCReads variable. Note that the cells having NEVER event (cells 4 and 6) have zeros for their TRUE coverage measures, since this event can never become true. We can 
also see that the event @F(mCardValid) (cell 2 in Table 10) was evaluated to true 3219 times and to false 18025 times when it was in scope (the mcStatus variable was equal to CheckCard).

Table 11: Cumulative coverage measures for the PACS Specification.

\begin{tabular}{|c|c|c|c|c|c|c|c|c|c|c|c|c|}
\hline cell & \multicolumn{2}{|c|}{1} & \multicolumn{2}{|c|}{2} & \multicolumn{2}{|c|}{3} & \multicolumn{2}{|c|}{4} & \multicolumn{2}{|c|}{5} & \multicolumn{2}{|c|}{6} \\
\hline coverage totals & TRUE & FALSE & TRUE & FALSE & TRUE & FALSE & TRUE & FALSE & TRUE & FALSE & TRUE & FALSE \\
\hline mcPIN & 3762 & 145220 & 1078 & 18885 & 242 & 4334 & 225 & 4351 & 60 & 996 & 57 & 999 \\
\hline mPINInput & 12 & 25043 & & & & & & & & & & \\
\hline mcStatus & 12685 & 75881 & 3758 & 45614 & 12 & 25803 & 5 & 35 & 2 & 31 & 3206 & 46166 \\
\hline cGate & 75 & 175071 & 175071 & 75 & & & & & & & & \\
\hline cGuardAlarm & 63 & 175083 & 175083 & 63 & & & & & & & & \\
\hline cGuardDisplay & 12443 & 162703 & 13 & 175133 & & & & & & & & \\
\hline cUserDisplay & 12434 & 162712 & 3206 & 171940 & 3758 & 171388 & 4 & 175142 & 14 & 175132 & 13 & 175133 \\
\hline tNumCReads & 10921 & 10323 & 3219 & 18025 & 13 & 6 & 0 & 19 & 1563 & 3183 & 0 & 4746 \\
\hline tNumPReads & 8 & 11 & 4 & 15 & 13 & 12 & 0 & 25 & 4 & 2 & 0 & 6 \\
\hline
\end{tabular}

From the Table 11 we can conclude that the uniform random testing performed by Lurch does not result in uniformity of the measured coverage across the model. For example, notice the difference in the coverage between the tNumCReads variable, and the tNumPReads variable. Although these two variables are very similar (one counts the number of incorrect card reads, and the other counts the number of incorrect PIN reads) and they have almost the same specification tables, tNumCReads has been more extensively covered than tNumPReads. This is caused by the fact that there is a sequence of specific events in the system that needs to be executed for a person to be validated. The probability of the uniform event generation to explore this sequence decreases exponentially with its length.

Thus, having parts of the specification receive low coverage makes it possible for errors not to be detected with the random testing approach. One solution might be to increase the depth of the generated random probes, or to give Lurch more time to exercise the model, or both. We can use the defined coverage measures as a stopping criterion to determine when to stop the random testing. 
We compared the induced coverage by the same event sequence generated by Lurch on the correct specification, and the faulty specifications with the idea that deviations might point out the undetected mutant specifications. Our findings were inconclusive - there were mutant specifications which had different degrees of deviation of the coverage from the correct one. We observed faulty specifications with the same coverage measures as the correct one, as well as faulty specifications with large difference in the induced coverage from the correct one.

Another approach to even-out the induced coverage by random testing tools like Lurch is to use different probabilities for input event generation instead of generating the input sequences uniformly. The strategy may represent the operational profile of the system under test. These probabilities can be modeled, for example, by using Markov models as described in [27].

In our case study with the PACS specification, there are two variables (mReset and mOverride) having low probability of occurrence in the real system (less than 1\%). However, they have a big effect of taking the system to the initial state. Therefore, during the course of our random testing, a change in one of these variables severely limits the depth of our exploration. As a crude experiment, we removed the generation of these events from the Lurch model, and that resulted in the successful identification of the remaining 8 mutants which were not detected in the initial test runs. 


\section{Chapter 6}

\section{Using Visual Interfaces for Validation}

A specifier, based on the informal specification from the user (or the domain expert), writes the formal system specification. This formal specification is based on the mental model (understanding of the system) that the specifier develops. It should be complete, minimal and consistent with respect to the domain expert's informal specification. After the mental model has been developed, it is mapped into a formal domain, usually written in some form of formal notation or a domain specific language (e.g. SCR [10][13], Z [31], Relational Specifications [47], etc.)

There are two sources of errors that can arise during the formalization process. The first source is the incomplete understanding of the informal specification (or the domain) resulting in the development of an incorrect system model. Second, the specifier can introduce errors by incorrectly mapping the (possibly correct) mental model into the formal domain. Both of these types of errors can be discovered to some extent by consistency and completeness checks and this procedure can be automated [9]. However, completeness and consistency are necessary, but not sufficient conditions in assuring that the developed model is correct. 
The mathematical formulas that express the system can be very complicated even if the system itself is of moderate size. System complexity increases the possibility that errors are included in the formal specification. The major difficulty lies in our ability to correctly express system behavior in terms of symbolic formulas. Research has been conducted on how to write and structure these formulas to support incremental understanding [9][50]. Our research further addresses this issue of improving the understanding of formal specifications, but in a graphical framework.

Another issue in the formalization phase is the domain expert's understanding of the formal specification. Often the domain expert does not have the expertise in the specific form of formal notation and cannot validate whether the formal specification really captures all the desired features of the system correctly. This is the validation problem. One of the proposed solutions is to have two specification groups that develop the specifications independently. The first group is responsible for development of the formal specification while the second group provides redundant information that can be used to validate the specification developed by the first group [47]. The drawback of this process is the fact that the domain expert is not involved in the validation, although he/she is the one that possesses the most complete knowledge of the required system behavior.

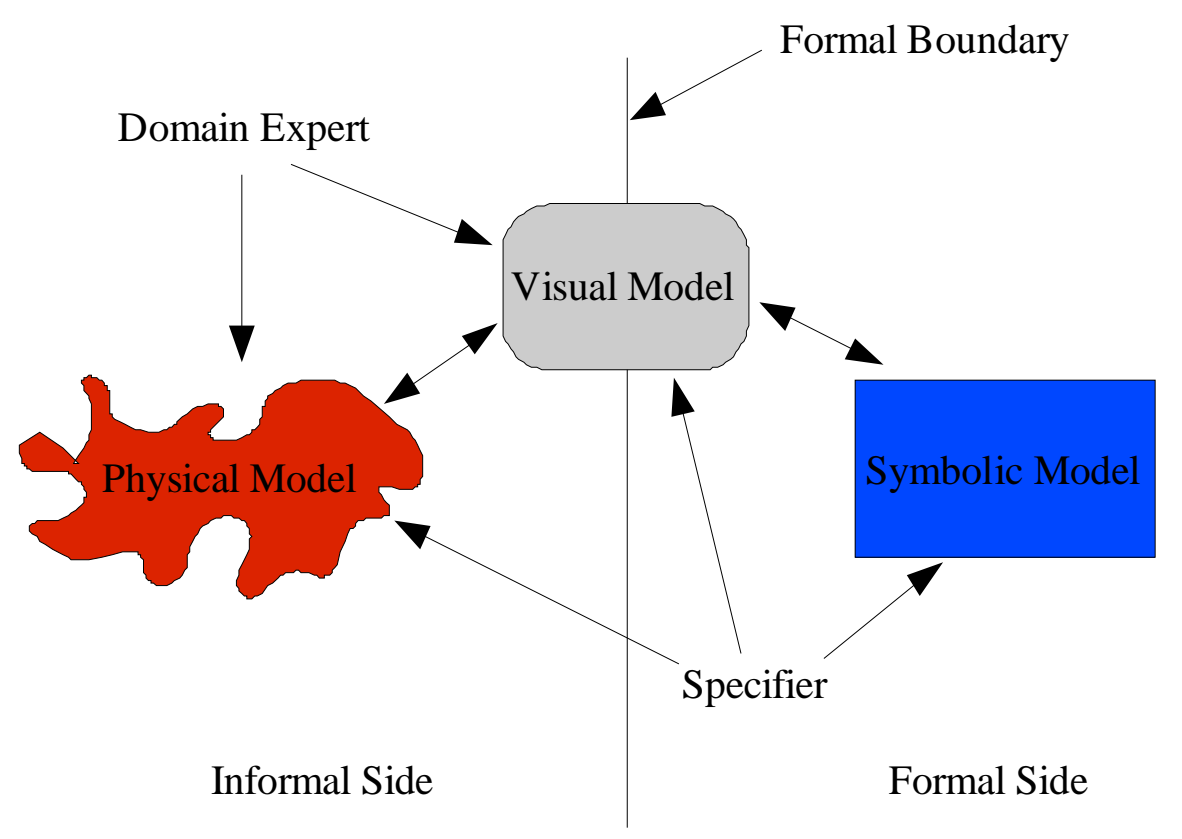

Figure 16: Using Visual Models for Validation. 
The domain expert can be included in the process of validating formal specification if the specification is rewritten in some easily understandable notation. Candidate notations are natural languages or visual models, but the automated rewriting is far from being simple.

By using graphical representation of the formal system model, the domain expert is included in the validation process. The specifier also benefits because the visual model increases his/her ability to construct the correct mental model and assists in the process of mapping this mental model to formal notations. Figure 16 depicts our intention to use the visual model as a bridge over the typical boundary between the domain expert's knowledge and the formal model, which (supposedly) captures it.

Many simulation researchers extensively explored the process of visualizing simulation models [11], and today visualization has become a critical component of simulation technology. Some of the aspects where visualization is found to be very beneficial in simulations (as identified in [39]) are the following:

- Verification and Validation - The logic model and the real-world behavior can be verified by watching the model. Animation, combined with sound statistical analysis, is an unmatched approach to evaluating how good a model really is.

- Understanding of Results - Often when the outcome from a model is not understood, the animation can provide insight. By watching the area(s) of interest, the modeler can "see" what is happening and understand how the dynamic behavior of the system affects the results.

- Communication of Results - Visualization is critical in communicating the outcome of a simulation to the non-technical audience. Watching a few minutes of visualization can eliminate hours of long tedious discussions. 


\subsection{Visualization of SCR specifications}

The SCR simulator has built in capabilities for visualization of specifications (see Figure 17). By using the developed visual objects given in the simulator palette, the users can visualize the variables used in a given SCR specification, and in a fast and efficient manner create a visual interface that can be used for the testing and validation of the specification. The palette is still work in progress, and it has limited number of visual objects that can be used (sliders, drop-down boxes, and picture changing elements). For the time being, their behavior is limited to displaying different pictures or text strings based on the values of the variables. Although limited, this behavior is enough to create rich visual interfaces for specifications where simple "point and click to change" interface is possible. However, if there is a need of more sophisticated display and interaction behavior where the user needs to drag or move some elements of the visual interface, we need to implement those objects in Java and add them to the interface, or find some existing implementations of similar objects and import them to the palette.

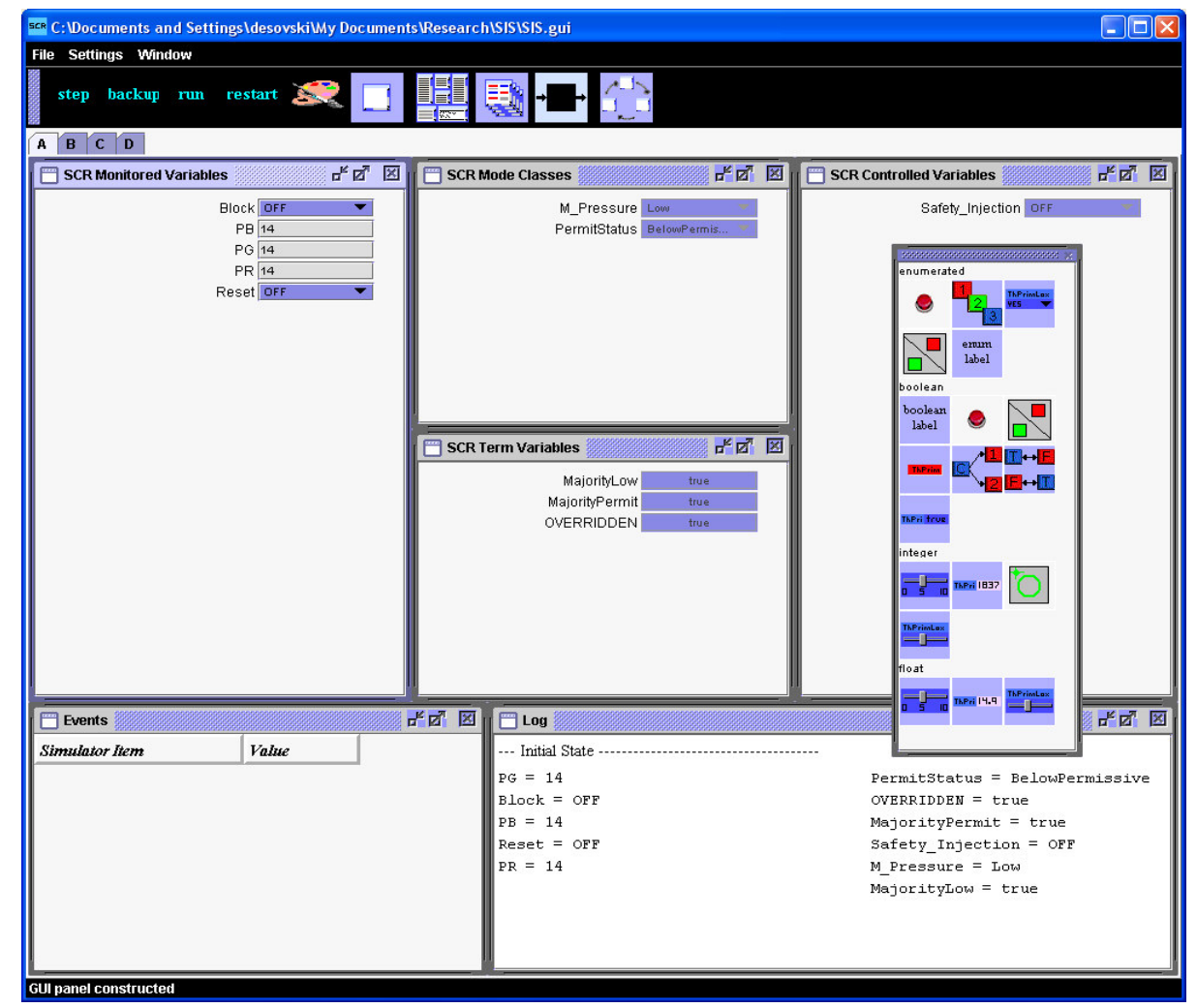

Figure 17: SCR simulator with the default interface for the Safety Injection System. 
Our research was focused on extending the SCR simulator. We investigated how existing and established visualization libraries can be used to create visual interfaces for SCR specifications, and how we can use the developed visual objects to enrich the existing simulator palette with minimal amount of programming required.

\subsubsection{Altia Design}

Altia Design [77] is a sophisticated graphics design environment. It is used for building animated, interactive graphical objects, which can be combined to create visual interfaces. The Altia Design Editor can be used to create the visualization and define the behavior and connections between the components and the external environment without any additional programming. Altia also provides libraries with great looking pre-built components. The design procedure in Altia Design is performed in four steps:

1. Drawing the graphics. The Graphics Editor can be used to draw components, import photo-realistic bitmap graphics from scanned images, or use the available components from the Altia libraries.

2. Define the behavior. Altia Animation tool is used to define the motion and appearance of objects by directly manipulating the object with the Graphics Editor. The Stimulus tool is used to define the interaction of the component with the user - mouse clicks, mouse motion, keystrokes, timer, etc. The Control tool is used to define more sophisticated behavior and component interaction.

3. Identify Properties. Once the components are built they can be packaged for reuse. The Properties tool is used to select number of properties that will be available to other users, such as color, font size, minimum and maximum.

4. Add connections. Once the objects are constructed and the visual interface is designed, it should be connected to the external simulator or code. Connection tool is used to define the connections between the different objects as well as the external environment. 


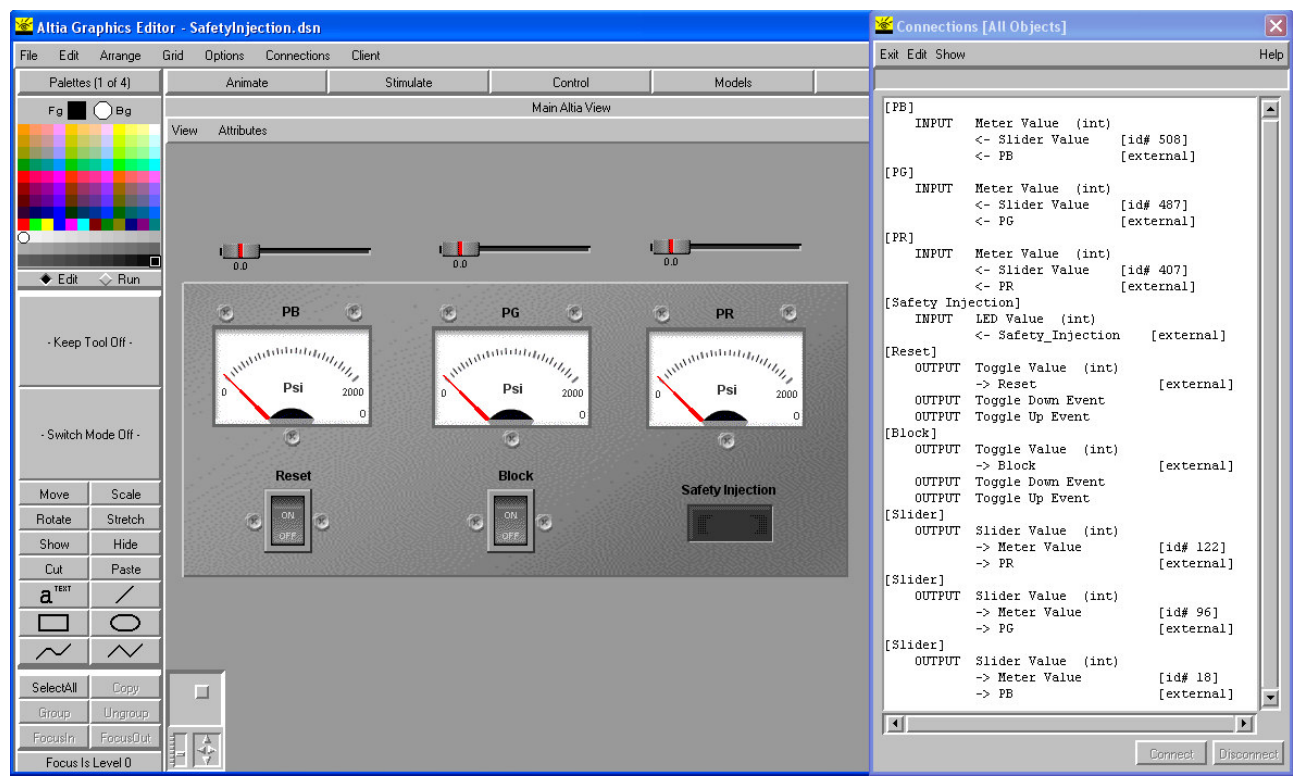

Figure 18: Designed SIS Visual Interface using Altia.

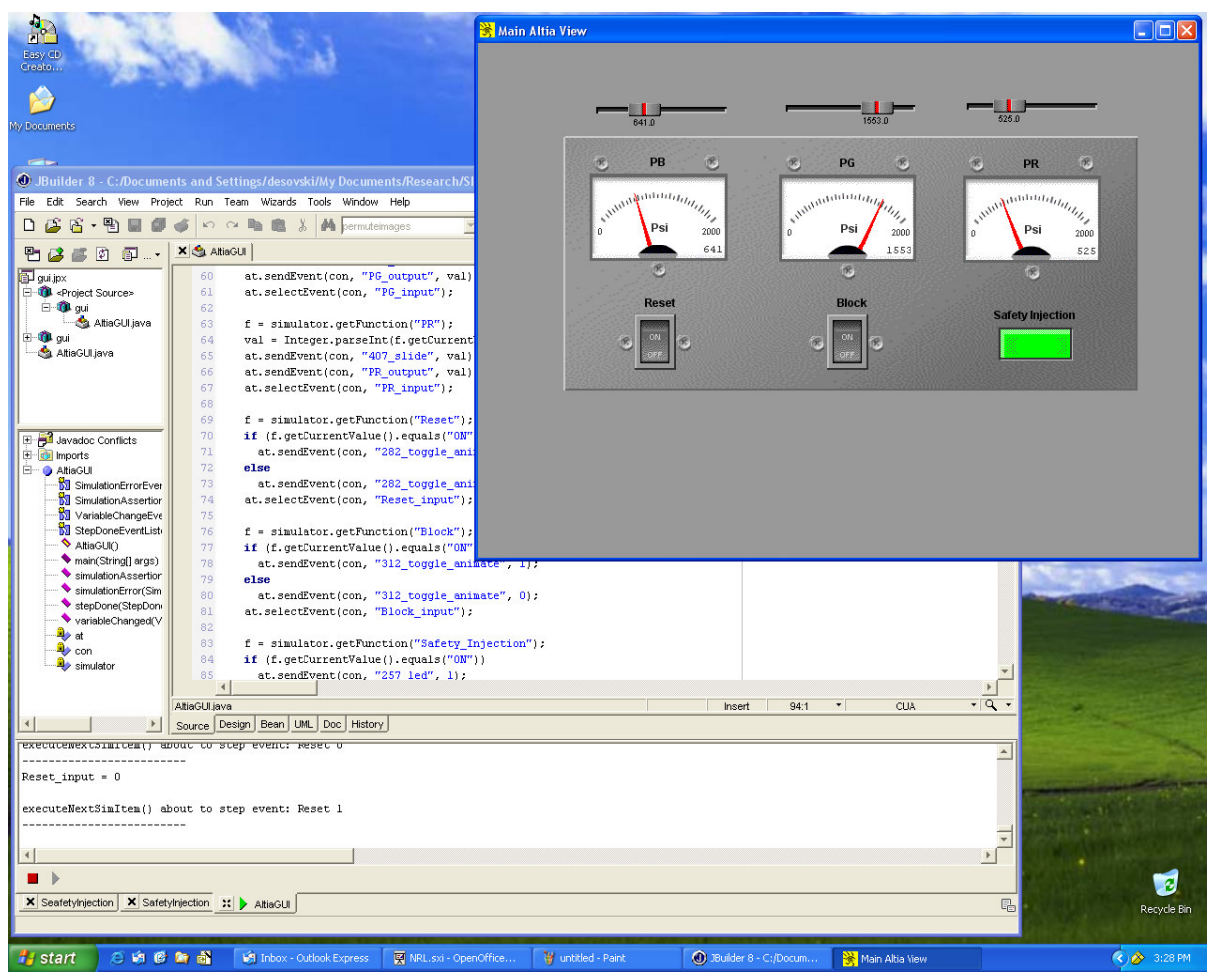

Figure 19: Interface code and Altia designed SIS panel. 
Figure 18 represents designed Safety Injection System (SIS) specification visual interface with the Altia Design and the defined connections between the components themselves as well as the external variables. We have used some of the existing objects in the Altia libraries (meter, slider, button, led, and some background objects) to create this visual interface. In order to connect the Altia Design with the SCR specification simulator, we have implemented a connection interface in Java, using the Altia Java APIs. Figure 19 represents a simulation of the SIS specification using the developed visual interface.

Altia has a large number of pre-existing components which have very nice photo-realistic design. However, changing the elements is complicated. Also, the designed objects can not be integrated with the SCR simulator palette and used in the SCR simulator - the designed visual interface must be run by the Altia runtime.

Although Altia has a large collection of very well designed visual components and the existence of Java APIs allows easy connection with the SCR specification simulator, we consider it only an external visual interface design tool that can be used to visualize SCR specification, and no integration with the SCR simulator is possible.

\subsubsection{JLOOX}

The JLOOX [78] visualization package has the similar components as the previously described Altia Designer. The JLooxMaker application is used to create visual designs without any additional programming. Visual objects can be designed using the drawing functions, created from imported pictures or external CAD programs, like AutoCad, or created from the pre-existing set of components. The designer defines the animations or possible user interactions with the components by using the appropriate tools. The number and the quality of the pre-existing components are not as good as the Altia package. However, the ease of creation of new dynamic objects and their modification for reuse is much better with $J L O O X$. Because $J L O O X$ is entirely written in Java it is possible for the created components to be encapsulated like Java beans and imported into the SCR simulator palette. 
The creation of dynamic objects (the objects that allow user interaction) is performed by selecting one of the DYNO object transformation (push button, toggle, slider, multiple state, dial, knob and digits are supported) and applying them to a set of components comprising the design of the object. Using some of the existing components and creating new ones we have developed the JLOOX SIS visual interface presented in Figure 9 together with the SCR interface code in the background.

Unlike the Altia interface the JLOOX-SCR interface code can be created to be very generic, without any references to the actual names of the objects in the design. The constructor of the SimulatorInterface class queries the SCR simulator for all functions contained in the SCR specification, and connects them with the appropriate JLOOX visual objects. The convention followed in this example is that a single variable corresponds to a singe visual object and their name is the same. However, this can be modified to allow multiple visual objects to represent the same value. By following a convention their name will have a prefix that is the same as the variable name and a suffix that will differentiate them. Consequently, the developed JLOOX-SCR interface code can be reused without any changes to drive all visual interfaces that have been built using the same convention.

It is important to note that the largest part of the code in the SimulatorInterface class is for handling type changes between different types of SCR variables and different types of visual components. We expect that further changes and improvements in this code will be needed and it would be useful to have a class that can convert between different types of variables just for this purpose.

The summary of our experience with $J L O O X$ is that it is much better suited for visualization of SCR specifications than Altia. Generic code can be developed to drive any design developed according to predefined naming conventions for the visual objects. Also, the created objects can be easily encapsulated in Java beans and imported into the SCR simulator palette. 


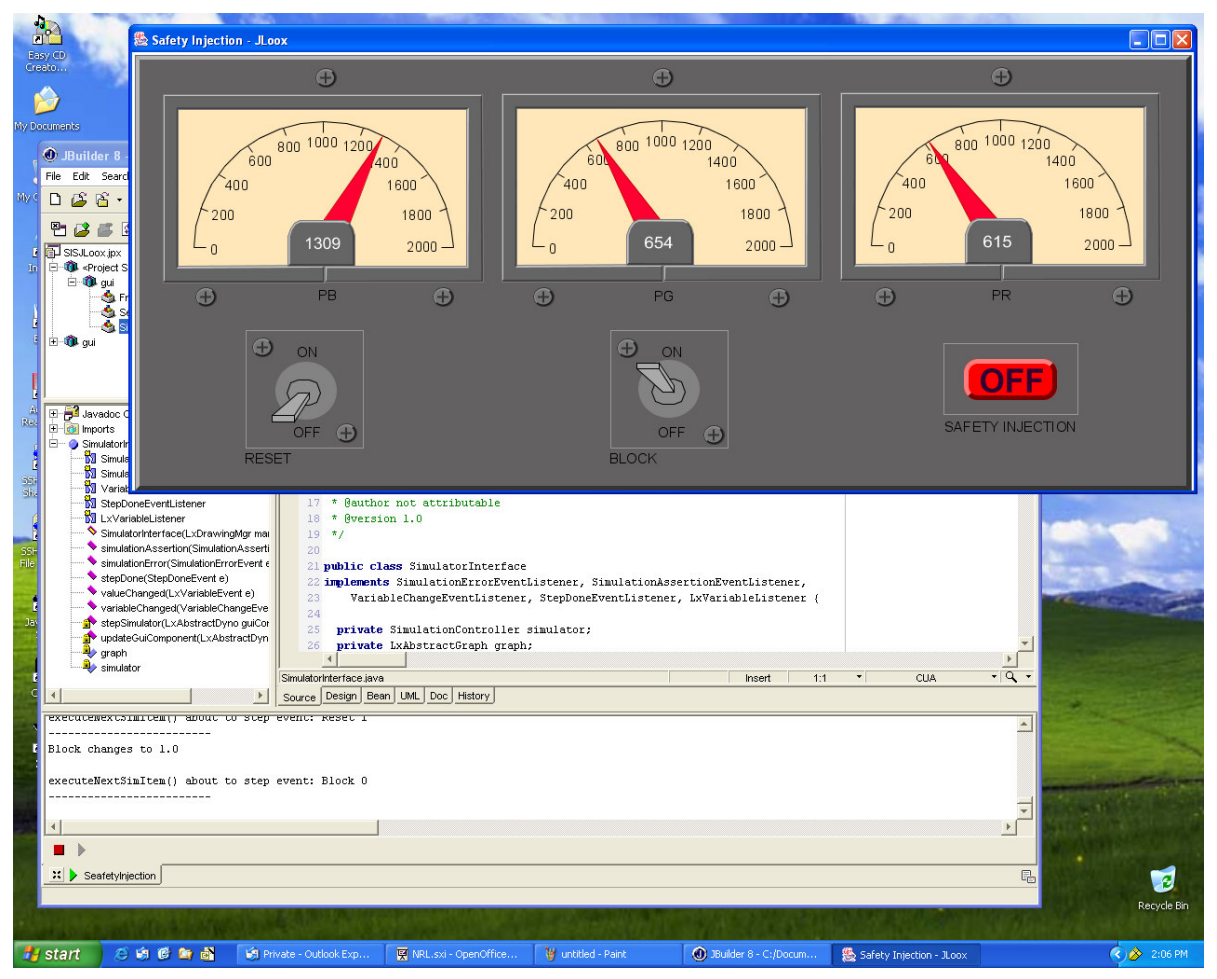

Figure 20: JLOOX SIS Visual Interface.

\subsubsection{ILOG JViews}

ILOG JViews [79] follows the same principles of visual interface creation as the previous two described packages. It has a Composer application which is used to create visual interface designs and dynamic objects (they are called "prototypes" in ILOG JViews). There is a set of pre-existing objects, but just like the JLOOX package, it cannot be compared with the Altia libraries. However, just like the JLOOX, the ease of the creation of the new objects and the ease of the modification of the existing objects is much better than the Altia Designer. Figure 10 displays the Composer with the meter object that we have created to mimic the JLOOX design.

The creation of prototypes is very straight-forward and intuitive by using the provided wizards. The designer specifies the properties of the prototypes and describes the graphical effects that are performed when the properties are changed, as well as the user interactions with the prototypes and their effect on the properties. Everything is done 
visually without any additional programming. Another very important feature of ILOG JViews package is that it provides automatic Java Bean encapsulation of the designer prototypes. Exported prototypes can be added to the SCR simulator palette. This is one of the features that we find particularly useful.

The connections between the different prototypes and to the external variables are created with the Connection tool by drawing a line between the connected components and assigning selecting the properties that will be communicated. The connections follow a publisher-subscriber paradigm; a change in a property that has subscribers will notify them and publish the new value. The connection to the external environment is done through special objects called ValueSources. There can be one or more ValueSources that can receive notifications when some of the prototype properties are changed by the user interaction, or send new values to the prototypes. Figure 21 presents our designed visual interface for the SIS specification with one ValueSource. Unfortunately, the ValueSources are placed in the top left corner of the design and cannot be moved around. For design clarity it would be best to group different types of SCR variables with particular ValueSource (e.g. ValueSource for monitored variables, ValueSource for controlled variables) and to be able to move them around because, usually, similar types of variables are placed close to each other in the visual designs.

We have implemented an interface for designed visualization to the SCR specification simulator. It is important to note that we do not need any additional object naming conventions, than we needed in the JLOOX case, because of the publisher-subscriber paradigm. Multiple objects can subscribe to the same SCR variable, or one object can subscribe to receive or send updates to multiple SCR variables.

The constructor of the SimulatorInterface class implements the opposite strategy of the one used in the JLOOX example for initialization and connection of the SCR variables with the visual interface, by first querying the visual design for the variables that are visualized. Depending on the size of the specification and the number of visualized variables this strategy might be better because usually not all SCR variables are represented by some objects in the visual interface. 


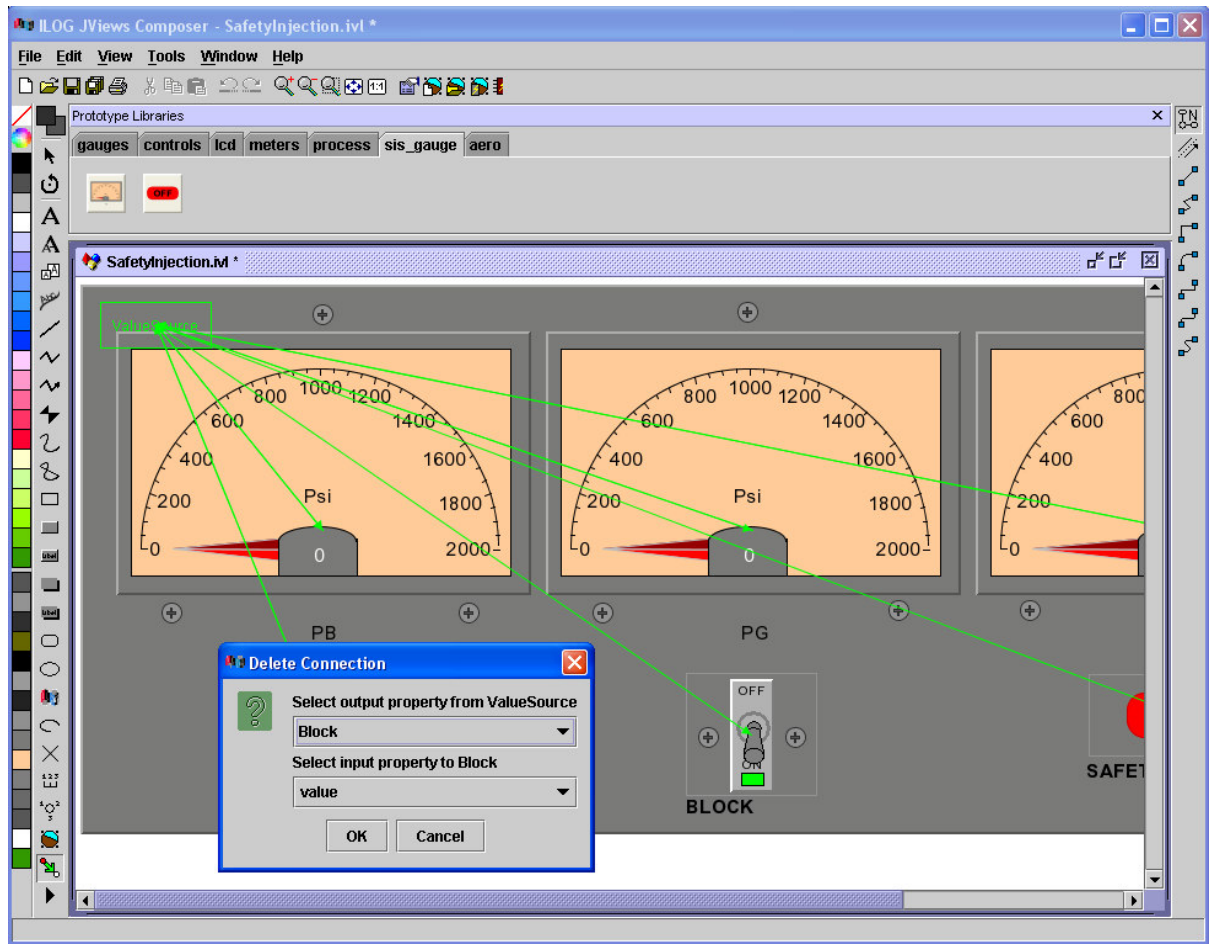

Figure 21: ILOG JViews SIS interface with the ValueSource connections.

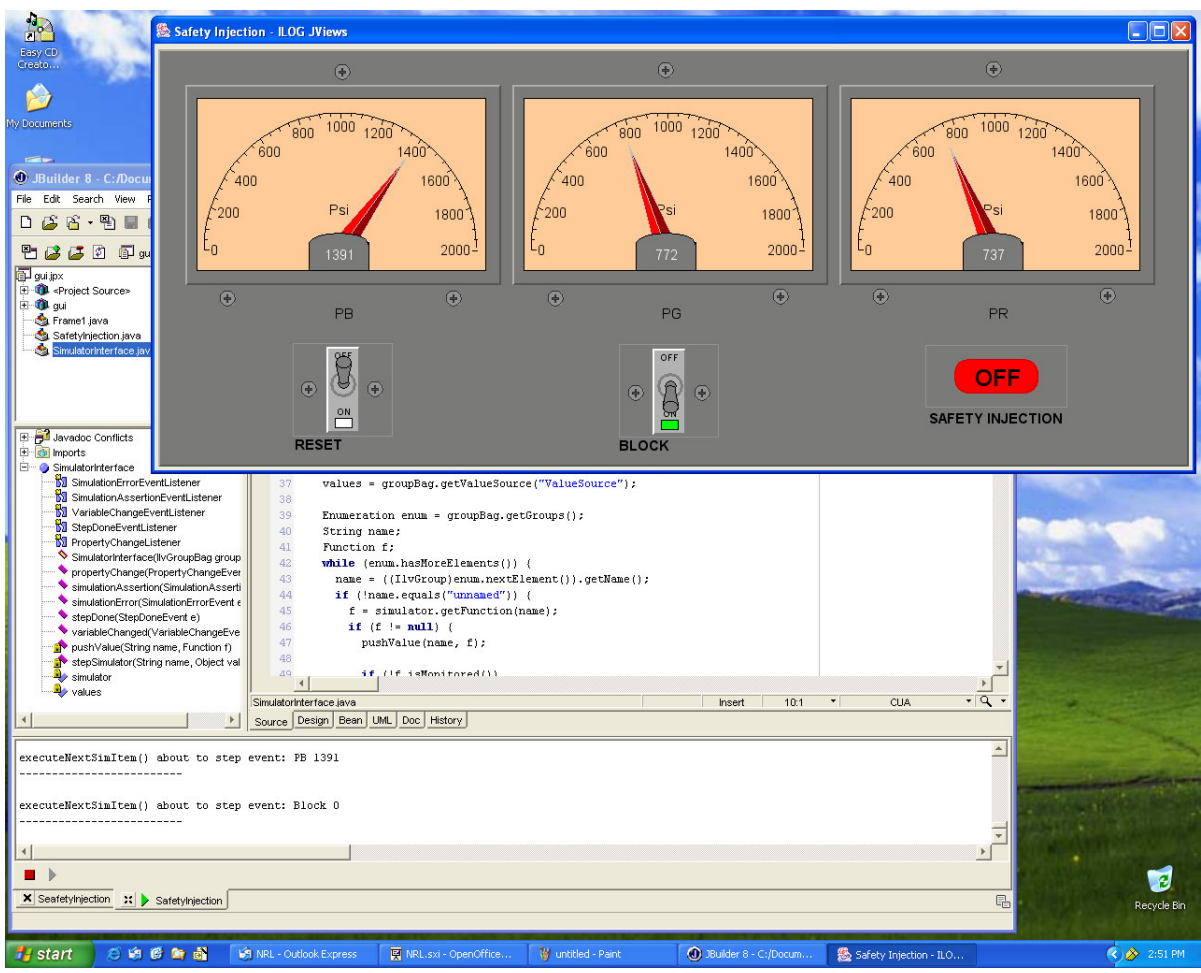

Figure 22: ILOG JViews SIS visual interface. 
Another important feature of the ILOG JViews package is that it provides a conversion class between different Java objects so that consequently the code for the SCR interface is much shorter and intuitive. Figure 22 presents the developed visual interface connected to the SCR generated Java code for the Safety Injection specification.

We used the Java Bean wizard and exported the meter object in order to add it to the SCR simulator palette. A small amount of programming work is needed here because the Java Beans components are unaware about the SCR environment. For any such Java Bean component (created with the ILOG JViews, purchased, or programmed) we will need to create a SCR wrapper that will allow the bean to interact with the SCR simulator environment. Figure 23 presents the SCR simulator palette with the ILOG JViews Meter object, and the created visual interface for the Safety Injection specification by using this object.

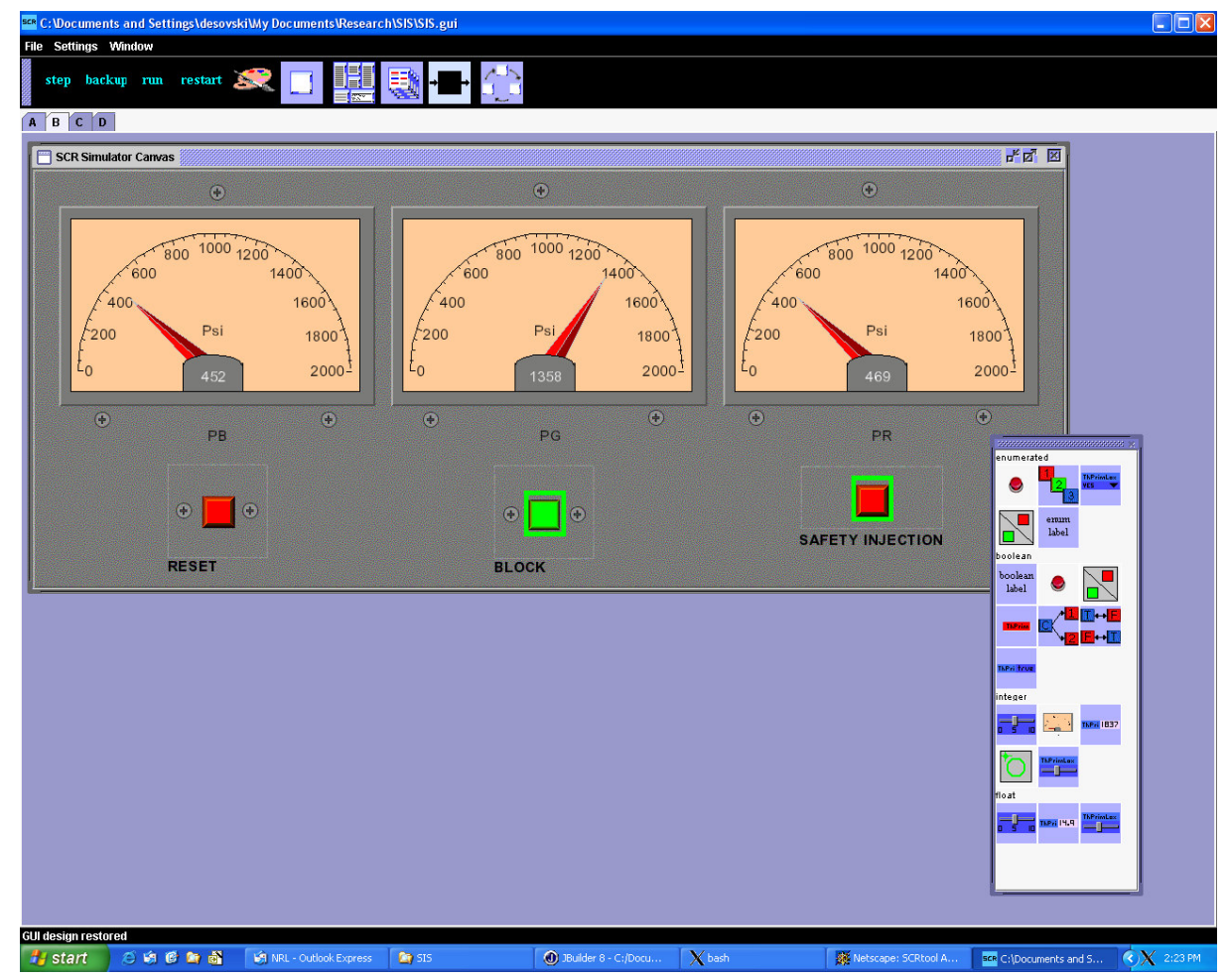

Figure 23: SCR simulator palette with the ILOG JViews Meter object. 


\subsection{Tracking the Coverage during Simulation}

The SCR simulator can execute the developed specification, thus providing us with testing capabilities. Although this feature has been implemented for a long time, testing has not been explored for the purpose of verification. This is mainly because the input spaces of moderate systems are enormous and the complete coverage of the input space of the model is impossible. Testing is used to prove the existence of errors, and cannot be used to prove their absence. Despite these problems, testing is the most used QA technique in the industry because it is easy to perform the actual tests. Recent papers in the area propose how to use model checking for automatic test case generation [4][5].

By using one of the developed visual interfaces, during testing we discovered a fault in the FPE (Fault Protection Engine) specification [4] developed in a joint project of NASA and NRL. Performing testing with these interfaces would allow the users to focus on the expected behavior of the system, providing significant evidence of the correctness of the specification. This research extends our previous research presented in [71] about using visualizations for validation of requirement specifications.

We implemented a prototype tool to track the test coverage for SCR specifications. In Figure 24 we present a visual interface for the Safety Injection specification running in the SCR simulator. While the user runs the simulator, the induced branch coverage is calculated and presented in the lower left corner. When the user completes a simulation, the detailed information is presented about which cases were not covered, and whether they are true of false coverage cases, for each table of the specification.

The coverage measures obtained during testing by the domain experts provide some information about the correspondence between the specification and the actual system in the following sense: if there are parts of the specification which have not been covered, it is possible that we have over-specified these aspects of the system. We would need to consult the domain expert whether these parts need to be removed, or if further test cases which are representative for these parts of the system can be developed. 
Our tool saves the executed test sequences, and they can be later used for certification of the developed executable code, or for the testing and validation of the specification as it evolves.

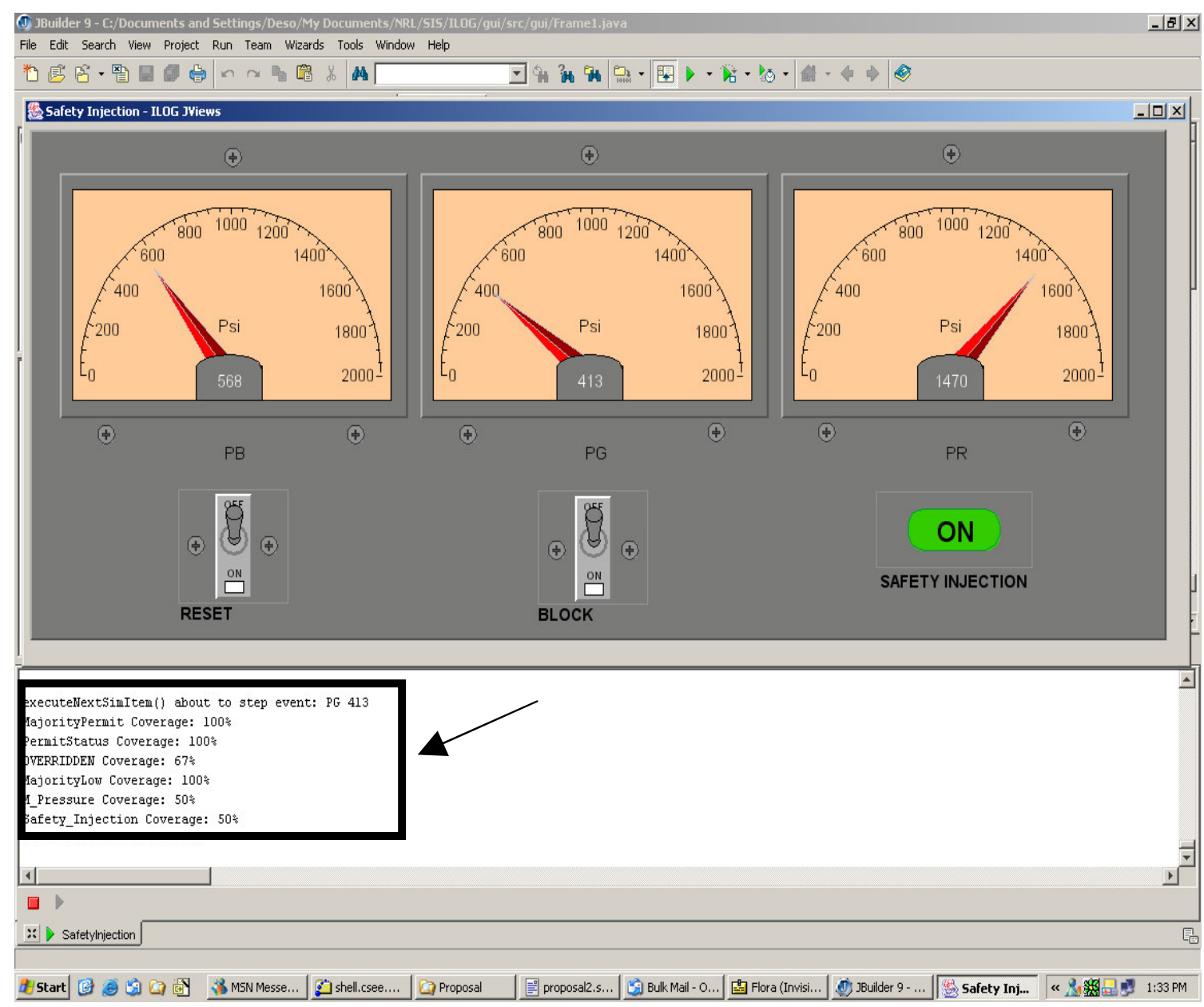

Figure 24: Prototype tool for tracking the coverage of the executed simulation. 


\section{Chapter 7}

\section{Case Study}

We demonstrate our methodology on an SCR specification of Personnel Access Control System (PACS), originally described in a prose requirements document from the National Security Agency [74]. The SCR specification (given in Appendix A.1.) had been originally developed as an example on how to write high quality formal requirements specification.

A high quality requirements specification must not only be easy to understand and change, precise, and unambiguous, it must also avoid implementation bias. In addition, it should be complete and consistent and organized as a reference document. Unfortunately, requirements specifications with all of these attributes are extremely rare. The original specification was developed focusing on two important aspects of a high quality requirements specification: the formulation of a set of system modes, which make the specification more concise and easier to understand, and the design of the specifications for ease of change. 


\subsection{PACS Description}

PACS checks information on magnetic cards and uses PIN numbers to limit physical access to a restricted area to authorized users. To gain access, the user swipes an ID card containing the user's name and Social Security Number (SSN) through a card reader. After using its database of names and SSNs to validate that the user has the required access privileges, the system instructs the user to enter a four-digit personal identification number (PIN). If the entered PIN matches a stored PIN in the system database, PACS allows the user to enter the restricted area through a gate. To guide the user through this process, the PACS displays messages on a single-line display screen. A security officer monitors and controls the PACS using a console with the second single-line display screen, an alarm, a reset button, and a gate override button.

To initiate the validation process, the PACS displays the message "Insert Card" on the user display. Upon detecting a card swipe it validates the user name and SSN. If the card is valid, the PACS displays "Enter PIN." If the card is unreadable or the information on the card fails to match the information in the systems database, the PACS displays "Retry" for a maximum of three tries. If after three tries the user's card is still invalid or there is no match, the system displays "See Officer" on both the user's display and the officer's display, and turns on an alarm (either a sound or a light) on the officer's console.

Before system operation can resume, the officer must reset the PACS by pushing the reset button. The user, who also has three tries to enter a PIN, has a maximum of five seconds to enter each of the four digits before the PACS displays the "Invalid PIN" message. If an invalid PIN is entered three times or the time limit is exceeded, the system displays "See Officer" on both the user and the officer display. After receiving a valid PIN, PACS unlocks the gate and instructs the user to "Please Proceed." After 10 seconds, the system automatically closes the gate and resets itself for the next user. 


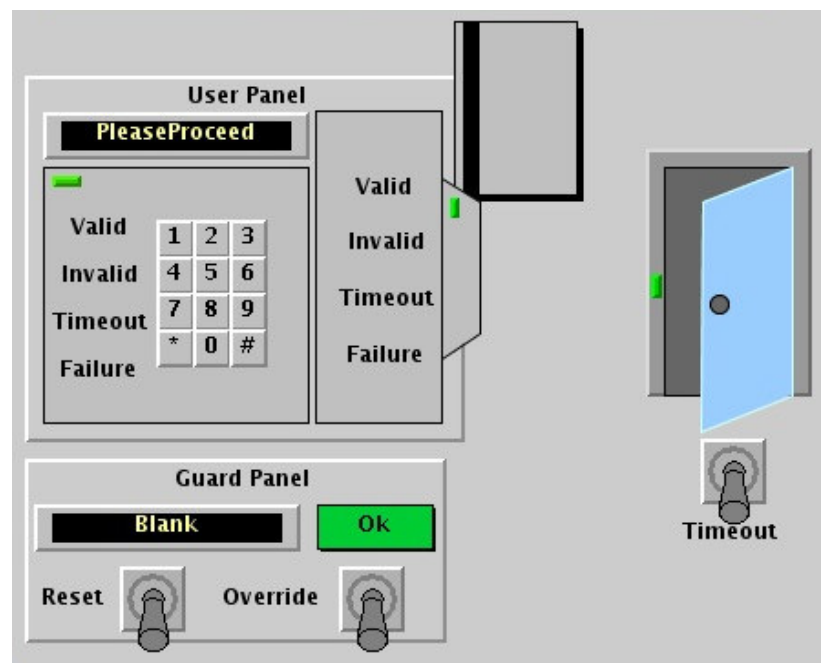

Figure 25: Visual Interface of the PACS specification.

Figure 25 presents a Visual Interface of the PACS system, which was used during the development of the specification.

\subsection{Applying the Decomposition Procedure}

The initial application of our decomposition algorithm on the PACS dependency graph was not successful in the sense that it did not identify the components we were expecting. Namely, from [74] we can see that PACS system has at least two components: the card reader and the PIN reader. Our decomposition heuristic could not detect these two components from the original specification leading to the conclusion that information hiding principles (advocated by Parnas et al. in [15]) have not been followed. After the careful review of the original specification and its dependency graph, we concluded that there are several factors limiting the decomposability of this specification:

- The behavior of the card reader is specified in the system status mode table, instead of being encapsulated as a separate component;

- The behavior of the PIN reader is separated from the system status mode table, however its information hiding can be improved (e.g. there are variables which are not completely encapsulated, adding unnecessary dependencies); 
- The specification contains two variables which directly affect most of the other variables in the system (mReset and mOverride). Although the behavior introduced by these two variables is simple (e.g. reset of all the variables in the system by mReset), they introduce additional dependency links that our algorithm cannot break.

To remedy the encapsulation problems, we refactored the original specification by applying the following principles:

1) Define term variables to represent the result of the operation of each component, thus encapsulating the internal component behavior.

2) Use mode classes for component specification.

3) Use the defined term variables when referring to the results of other components (do not break the encapsulation and information hiding by using internal component variables).

4) Avoid the use of global variables.

To break the dependencies on the global variables, we had to remove them from the specification. There are several ways this could be done in the future - we can denote these variables as global and relax the cost of the dependencies links that are connected to them, allowing the decomposition algorithm to break these links.

For the PACS specification, we defined term variables $t$ CardValid and $t$ PINValid to encapsulate the behavior of the two components. Both of them have the same domain \{Unknown, Yes, No, Error $\}$ denoting that, for example, tCardValid is either Unknown we do not currently know the result of the card entry and validation, Yes - the card is successfully swiped and validated, No -the card is not valid, or Error - the user exceeded the number of allowed non-valid swipes. The Error state allows us to encapsulate the tNumCReads variable, counting the number of non-valid swipes within the component. For each component we used a mode class (mcCard and $m c P I N)$, which simplifies the 
specification of their behavior. The system mode status table mcStatus and the other external variables to the components are changed only to refer to the defined terms.

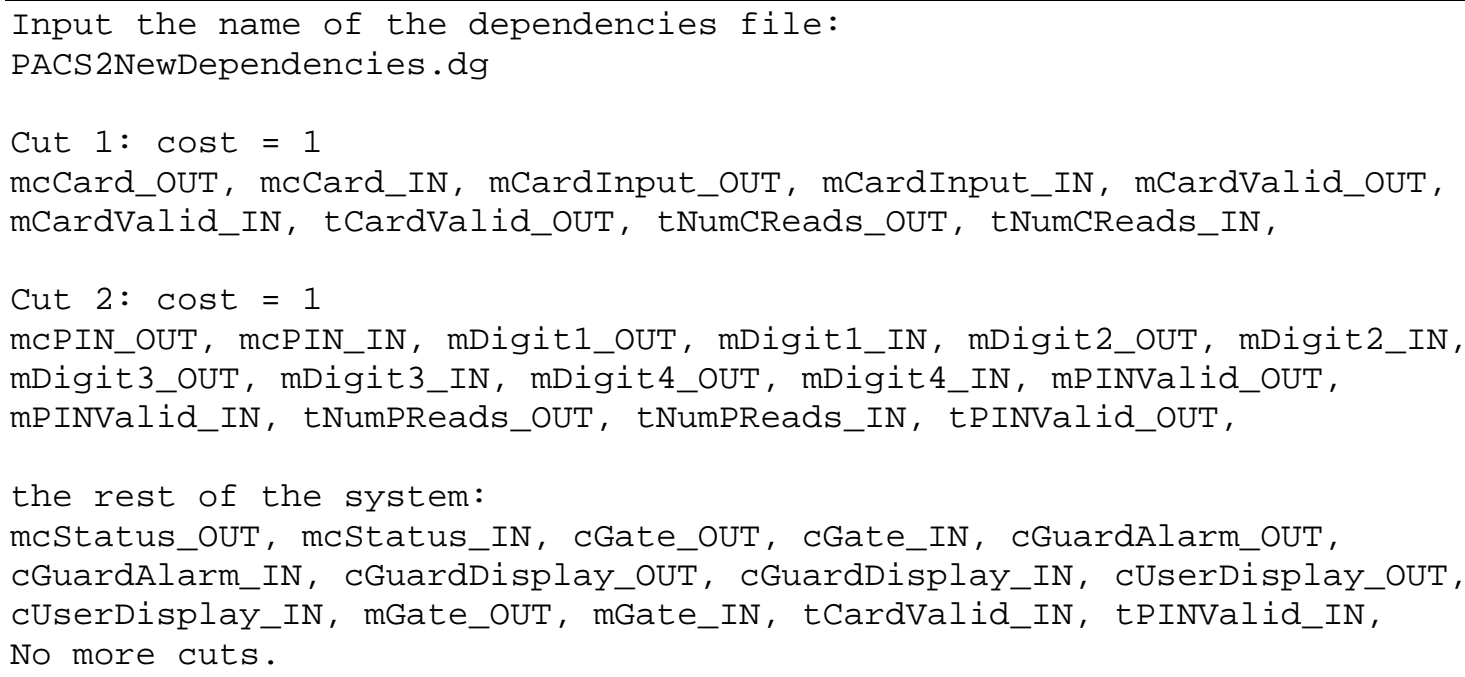

Figure 26: Decomposition of the refactored PACS specification.

After this refactoring, our decomposition algorithm correctly identified the two components of the system, leaving only the control component (Figure 26).

These changes in the operational part of the specification imply only small changes in the specification of properties. Because we removed the mReset and mOverride variables, we had to remove one of the properties which referred to these variables. The rest of the properties were either unchanged or only had replacement of the mCardValid or mPINValid monitored variables, with the corresponding term variables $t$ CardValid and tPINValid.

We used the SPIN model checker with default options and increased the depth limit to 180000 in order to achieve complete verification to verify all properties for the complete refactored PACS specification (Appendix A.2.). This took 35.405 seconds and required 553.342 MB of memory on a machine with Pentium M $1.5 \mathrm{GHz}$ processor and $1 \mathrm{~GB}$ of RAM. 


\subsection{Applying the compositional verification strategy}

Step 1 of our verification strategy gives the following partitioning of the variables:

$V_{1}=\{m$ CardValid, mCardInput,$t N u m C R e a d s$, mcCard,$t$ CardValid $\}$,

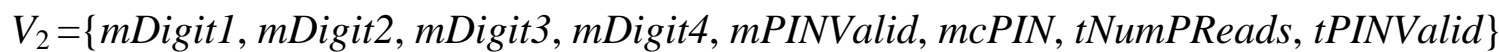

$V_{3}=\{m$ Gate, mcStatus,$c$ GuardAlaram,$c$ GuardDisplay, $c$ Gate,$c$ UserDisplay $\}$

In step 2, we derived the three components presented in Figure 27, Figure 28, and Figure 29. In the figures, we omitted the variables on which the components do not have explicit dependency. Since the properties of interests for each component are only those that depend on the variables within the component, the variables from the set $V-V_{i}$ are going to be abstracted by applying the abstraction method 1 described in Section 4.1.

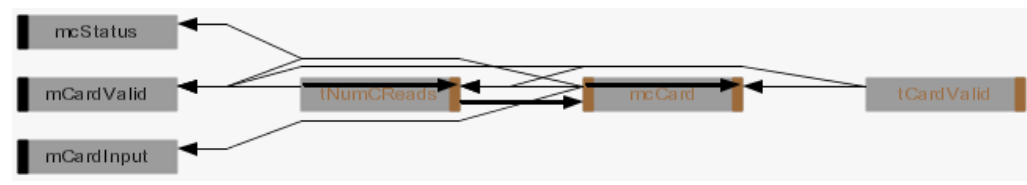

Figure 27: The Card reading component.

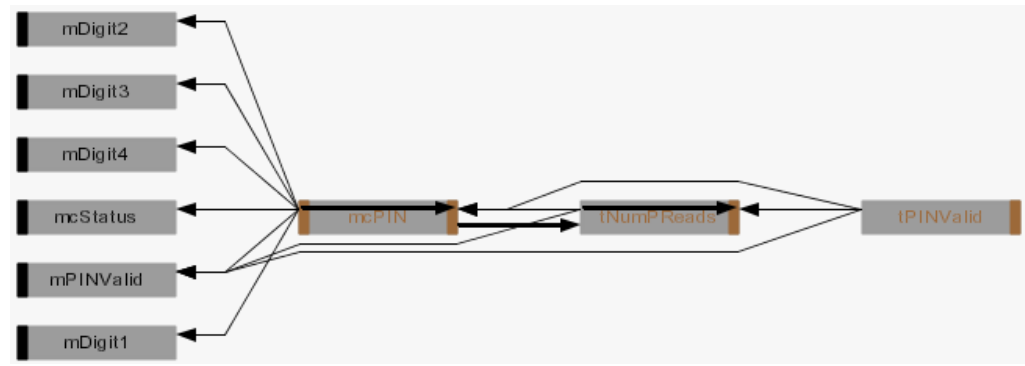

Figure 28: The PIN-Reading component. 


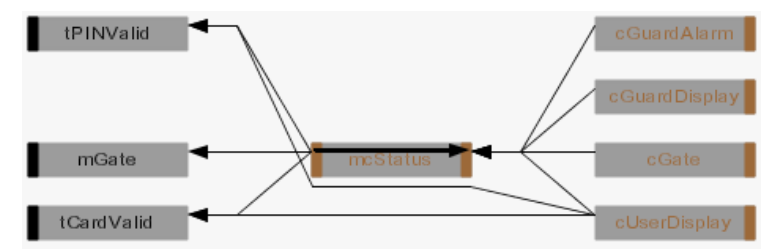

Figure 29: The Control component.

Next, in step 3 we need to prove the properties that hold for the identified components.

The verification of the Control component (Figure 29) with SPIN took 0.093 seconds and required $2.827 \mathrm{MB}$ of memory, establishing the following invariants for the system:

AlarmStatus: $(\mathrm{cGuardAlarm}=$ On $)<=>(\mathrm{cUserDisplay}=$ SeeOfficer $)$

CardSuccess: ((cUserDisplay = InsertCard OR cUserDisplay = Retry) AND mcStatus = CheckCard AND @ T $($ tCardValid = Yes $))=>($ UUserDisplay' = EnterPIN $)$

GateStatus: $(\mathrm{cUserDisplay}=$ PleaseProceed $) \Leftrightarrow=($ cGate $=$ Open $)$

PINSuccess: $((\mathrm{cUserDisplay}=$ EnterPIN OR cUserDisplay $=$ InvalidPIN $)$ AND mcStatus $=$ CheckPIN AND @ T(tPINValid = Yes $))=>($ cUserDisplay' $=$ PleaseProceed $)$

Safety: (cUserDisplay $=$ SeeOfficer $) \longleftrightarrow=($ cGuardDisplay $=$ SeeOfficer $)$.

The verification of the Card-Reading component takes 0.062 seconds and requires 2.724MB of memory, establishing the following invariants for the system:

CardErrors: $($ mcStatus $=$ CheckCard AND @ T $($ tCardValid $=$ No $))=>\left(\mathrm{tNumCReads}^{\prime}=\right.$ tNumCReads + 1)

NumCardErrors: (tNumCReads <= MaxCardError).

Similarly, the verification of the PIN-Reading component takes 2.327 seconds and requires $55.320 \mathrm{MB}$ of memory, establishing the following properties as invariants: 
NumPINErrors: (tNumPReads $<=$ MaxPINError)

PINErrors: $($ mcStatus $=$ CheckPIN AND @ $\mathrm{T}(\mathrm{tPINV}$ alid $=$ No $)) \Rightarrow\left(\mathrm{tNumPReads}^{\prime}=\right.$ tNumPReads +1$)$.

We should note that the verification of the PIN-Reading component requires most resources, because its state space is larger. Also, note that the demonstrated CardErrors and PINErrors invariants are stronger than the ones included in the system specification in Appendix A.2.

Consequently, by applying step 3 of our verification strategy, we verified 9 out of the 14 invariants, in total of 2.482 seconds and requiring maximum 55.320MB of memory, which is for an order of magnitude more efficient than performing verification on the complete system.

The rest of the properties include variables that span across more than one component. We were not successful in applying the deduction rules in step 4 to prove the remaining 5 properties, partly because the automated translation from the SCR toolset to Promela currently does not handle assumptions. The results in using Salsa and SMV suggest that the problem in verification of these 5 properties is the over-approximation introduced by the decomposition. We would need to come up with additional invariants or rewrite these properties, but that was not our goal in this study.

CardDisplay1: (tNumCReads $>0$ AND tNumCReads $<$ MaxCardError) $\Leftrightarrow$ $(\mathrm{cUserDisplay}=$ Retry $)$

CardDisplay2: $(\mathrm{tNumCReads}=$ MaxCardError $)=>(\mathrm{cUserDisplay}=$ SeeOfficer $)$

PINDisplay1: (tNumPReads $>0$ AND tNumPReads < MaxPINError) $\Leftrightarrow$ (cUserDisplay $=$ InvalidPIN)

PINDisplay2: $(\mathrm{tNumPReads}=$ MaxPINError $)=>(\mathrm{cUserDisplay}=$ SeeOfficer $)$

PINEntry: $(@ \mathrm{C}(\mathrm{tPINValid})=>(\mathrm{mcStatus}=\mathrm{CheckPIN}))$ 
By using step 5 of our strategy and creating unions of components, we are successful in verifying the rest of the properties. Combining the Card-Read and Control components allows verification of the CardDisplay1 and CardDisplay2 properties in 0.124 seconds with $3.544 \mathrm{MB}$ of memory. More demanding is the union of the PIN-Read and Control components, which allows verification of the remaining 3 properties in 16.734 seconds and $273.060 \mathrm{MB}$ or memory. Table 12 summarizes the results of the case study.

Table 12: The resources needed and number of verified properties for each component.

\begin{tabular}{|l|c|c|c|}
\hline \multicolumn{1}{|c|}{ Module } & Time (seconds) & Memory (MB) & Properties Proven \\
\hline Complete PACS & 35.405 & 553.342 & 14 \\
\hline Control & 0.093 & 2.827 & 5 \\
\hline Card Read & 0.062 & 2.724 & 2 \\
\hline PIN Read & 2.327 & 55.320 & 2 \\
\hline Card Read + Control & 0.124 & 3.544 & 2 \\
\hline PIN Read + Control & 16.734 & 273.060 & 3 \\
\hline
\end{tabular}




\section{Chapter 8}

\section{Conclusions and Future Work}

In order to perform verification and validation of complex system specifications, it is beneficial to identify the sub-components, apply abstraction and compositional verification methods. Our approach is based on the hypothesis that specification components can be automatically identified at the points that have minimal coupling with the rest of the system. In the case of SCR specifications, these points are presented by the variables in the specification, and coupling appears in the dependency graph. Applying the minimum cut algorithms, we can identify these points in the graph and perform specification decomposition.

We observed that our hypothesis holds true for specifications that follow the principle of information hiding. The proposed algorithms are polynomial and therefore applicable to large specifications. We demonstrated that our algorithm automates the abstraction procedure for removal of detailed monitored variables in the SCR specifications.

We presented the theoretical framework for compositional verification of the SCR specification properties. The identified deduction rules are sound, but incomplete in general, possibly requiring additional inductive invariants that should be used to prove some of the system invariants. Although we are focusing on the SCR requirements 
model, the same approach should be applicable to similar formal models (e.g. Reactive Modules [69]), and this is one of the subjects for future research.

Assuming that the specification under review is decomposable, we proposed verification strategy that provides significant reductions in the required time and memory. Each derived component represents an abstraction of the complete system; however, it usually allows additional behaviors that might invalidate the properties we would like to prove. The min-cut decomposition approach minimizes the dependency of a single component on external variables. Our approach considers components of the specification (i.e. sets of variables) which have low coupling and limited information exchange with the rest of the specification. We specify properties at component level, prove that they are system invariants, and use them in the certification process. This does not always work, because of the additional behavior that the abstract components might have when considered by themselves. The invariants proven this way can be used to prove additional invariants by using the strengthening compositional and assume-guarantee rules.

During our experiments with the PACS system, we have identified the basic principles that need to be followed when writing decomposable SCR specifications. They echo the long time advocated principles of modularity, encapsulation, and information hiding in the software development process:

1) Define component border variables that will represent the result of the operation of each component, thus enabling encapsulation of the internal component behavior. In SCR models, these variables are represented as term variables.

2) Use variables that capture the internal state of the system modules in order to achieve specification modularity and ease of change. In SCR models, these variables are represented as mode class variables.

3) Use the defined border variables (from 1) when referring to the results of other components (do not break the encapsulation by using internal component variables). 
4) Avoid the use of global variables, or break the dependencies caused by them.

The results of the PACS case study demonstrate the advantages of decomposable specifications. Following the proposed verification strategy we achieved significant reduction in time and memory required for automated verification. The strategy allows us to perform the verification component by component, and combine the obtained results.

\subsection{Future Work}

In the future, we would like to further demonstrate the effectiveness of our componentbased verification methodology by applying it to larger software models. Important aspect of this work would be to formally identify the design principles that lead to decomposable and easily verifiable formal software models.

We presented our methodology and tools for random testing of formal software models. Although simple, random testing approach is effective in the early phases of formal modeling. It identifies faults and inconsistencies between the operational and the property-based description of the system. Because of the inherent incompleteness of the random testing, we need to augment our tools by using complete verification tools, like SPIN, in the later phases of the model development. We, however, note that this is not always feasible due to the large state spaces of practical software systems; current complete model-checking approaches often hit the state explosion barrier and cannot be applied. For this purpose, we plan to investigate the proposed test coverage metrics for SCR formal models and, if necessary, develop comprehensive coverage metrics. One direction is to use different input generation strategies, which would explore the structure of the model more uniformly. The other goal is to modify our random search methodology to automatically explore uncovered parts of the model leading to higher assurance in the correctness of the model under study.

Current requirements validation techniques do not address the problem of demonstrating the correctness of the formalization phase satisfactorily. This problem is especially 
significant in the realm of high assurance systems. Current techniques do not provide friendly interfaces for domain experts although they have the most complete knowledge of the system being built. We believe that validation techniques based on reasoning about symbolic system representation do not take advantage of the human cognitive process. These problems can be addressed in future by having a suitable visual interface in which symbolic formulas can be projected, visualized, and validated.

The SCR method addresses the validation problem with a number of approaches. The underlying model is easy to understand and uses compositional approach in building the model. The automated consistency and completeness checks ensure that the defined functions are deterministic and well defined. Different model checkers, theorem provers, and the SCR simulator can be used for the validation of the developed specifications.

There are several benefits in this approach. Creating a visual interface will unavoidably increase the time/resources required in the formalization phase, and thus if some users of the tool already have experience in using some visualization library, they can reuse this knowledge and the set of developed visual components. We also believe that the benefit of having visual interface in the validation process will compensate the initial higher cost. The developed specification visualizations can be connected to be driven not just by the SCR specification, but also by different implementations or specifications of the same system, and thus be used in cross validation.

Another subject that we would like to explore in the future is the input domain partitioning based on SCR specifications. If we are given an internal state of an SCR model, it induces a partition in the input space. In other words, it should be possible to generate input state partitions that do (or do not) change the value of the state variables. This result may lead towards more efficient testing strategies for SCR models by obtaining complete equivalence partitioning of the input space. 


\section{References}

[1] A. Biere, A. Cimatti, E. Clarke, Y. Zhu, "Symbolic model checking without BDDs", In Proceedings of the Workshop on Tools and Algorithms for the Construction and Analysis of Systems(TACAS'99), LNCS. Springer-Verlag, 1999.

[2] A. Biere, A. Cimmati, E. M. Clarke, O. Strichman, Y. Zhu, "Bounded Model Checking". Advances in Computers, Vol. 58, Academic Press, 2003.

[3] A. Groce, W. Visser, "Heuristic Model Checking for Java Programs", SPIN Workshop on Model Checking of Software, 2002.

[4] Allen Nikora, Constance L. Heitmeyer, "Automated Specification-Based Test Case Generation using SCR". International Workshop on Software Engineering for High Assurance Systems (SEHAS 2003) held in conjunction with International Conference on Software Engineering (ICSE 2003), Portland, Oregon, USA, May 2003.

[5] Angelo Gargantini and Constance Heitmeyer, "Using Model Checking to Generate Tests from Requirements Specifications". In proceedings of joint 7th European Software Engineering Conference and 7th ACM SIGSOFT International Symposium on Foundations of Software Engineering (ESEC/FSE99), Toulouse, FR, Sept. 6-10, 1999.

[6] Bojan Cukic, Hany H. Ammar, Khalid Lateef, "Identifying High-Risk Scenarios of Complex Systems Using Input Domain Partitioning". In proceedings of the $9^{\text {th }}$ International Symposium on Software Reliability Engineering (ISSRE'98), 4-7 November 1998, Paderborn, Germany, pp. 164-173.

[7] C. A. R. Hoare, "An Axiomatic Basis for Computer Programming". Communications of the ACM, Vol. 10, No. 10, October 1969, pp. 576-583.

[8] C. Chekuri, A. Goldberg, D. Karger, M. Levine, C. Stein, "Experimental study of minimum cut algorithms". Proceedings of the $8^{\text {th }}$ Annual ACM-SIAM Symposium on Discrete Algorithms (SODA'97), New Orleans, 1997, pp. 324-333.

[9] C. Heitmeyer, J. Kirby, B. Labaw, R. Bharadwaj, "SCR*: A Toolset for Specifying and Analyzing Requirements". In proceedings of the 10th Conference on ComputerAided Verification, (CAV'98), Vancouver, Canada, 1998.

[10] C. L. Heitmeyer, R. D. Jeffords, B. G. Labaw, "Automated consistency checking of requirements specifications". ACM Transactions on Software Engineering and Methodology, Vol. 5, No. 3, pp. 231-261, April-June 1996. 
[11]C. L. Swider, K. W. Bauer Jr., T. F. Schuppe, "The Effective Use of Animation in Simulation Model Validation". In proceedings of 1994 Winter Simulation Conference, Lake Buena Vista, FL, December 11-14, 1994.

[12] Constance Heitmeyer, James Kirby Jr., Bruce Labaw, Myla Archer, Ramesh Bharadwaj, "Using Abstraction and Model Checking to Detect Safety Violations in Requirements Specifications". IEEE Transactions on Software Engineering, Vol. 24, No. 11, November 1998, pp. 927-948.

[13] Constance Heitmeyer, Myla Archer, Ramesh Bharadwaj, Ralph Jeffords, "Tools for constructing requirements specifications: The SCR toolset at the age of ten". International Journal of Computer Systems Science and Engineering, January 2005, Vol. 5, pp. 95-114.

[14]D. J. Richardson, S. L. Aha, T. O. O'Malley, "Specification-based test oracles for reactive systems", Proceedings of the $14^{\text {th }}$ International Conference on Software Engineering (ICSE'92), 1992.

[15]D. L. Parnas, P. C. Clements, D. M. Weiss, "Modular Structure of Complex Systems", Proceedings of the $7^{\text {th }}$ International Conference on Software Engineering, pp. 408-417, 1984.

[16]D. Owen, B. Cukic, T. Menzies, "An Alternative to Model Checking: Verification by Random Search of AND-OR Graphs Representing Finite-State Models". In proceedings of $7^{\text {th }}$ IEEE International Symposium on High Assurance Systems Engineering, Tokyo, Japan, October 2002.

[17]D. Owen, T. Menzies, "Lurch: A Lightweight Alternative to Model Checking", Proceedings of the $15^{\text {th }}$ International Conference on Software Engineering and Knowledge Engineering, 2003.

[18] Dick Hamlet, Dave Mason, Denise Woit, “Theory of Software Reliability Based on Components", Proceedings of the International Conference on Software Engineering (ICSE'01), 2001.

[19] Doron A. Peled. Software Reliability Methods. Springer-Verlag, 2001.

[20]E. M. Clarke, D. E. Long, K. L. McMillan, “Compositional Model Checking”. In proceedings of the $4^{\text {th }}$ IEEE Symposium on Logic in Computer Science, pp 353-362, 1989.

[21]E. M. Clarke, E. A. Emerson, A. P. Sistla, "Automatic verification of finite-state concurrent systems using temporal logic assertions". In proceedings of the $10^{\text {th }}$ ACM Symposium on Principles of Programming Languages, Austin, Texas, January 1983.

[22]Edmund M. Clarke Jr., Orna Grumberg, Doron Peled. Model Checking. The MIT Press, 2001. 
[23]Edmund M. Clarke, Orna Grumberg, David E. Long, "Model Checking and Abstraction". ACM Transactions on Programming Languages and Systems, pp. 1512-1542, Vol. 16, No. 5, September 1994.

[24]Elaine Weyuker, Tarak Goradia, and Ashutosh Singh, "Automatically Generating Test Data from a Boolean Specification". IEEE Transactions on Software Engineering, Vol. 20, No. 5, May 1994.

[25]Elanie J. Weyuker, Stewart N. Weiss, Dick Hamlet, "Comparison of Program Testing Strategies". In proceedings of ACM SIGSOFT International Symposium on Software Testing, Analysis and Verification, Victoria, British Columbia, Canada, 1991.

[26] Elizabeth Leonard, Constance Heitmeyer, "Program Synthesis from Formal Requirements Specifications using APTS". Higher-Order and Symbolic Computation 16 (1-2): 63-92, Kluwer Academic Publishers, March - June, 2003.

[27] J. A. Whittaker, J. H. Poore, "Markov Analysis of Software Specifications", ACM Transactions on Software Engineering and Methodology, vol. 2, no. 1, 1993.

[28] J. D. Musa, A. Iannino, K. Okumoto. Software Reliability: Measurement, Prediction and Application. McGraw-Hill, New York, 1987.

[29] J. Edmonds, R. M. Karp, "Theoretical Improvements in Algorithmic Efficiency for Network Flow Problems". Journal of the ACM, Vol. 19, 1972, pp.248-264.

[30] J. H. Andrews, L. C. Briand, Y. Labiche, "Is Mutation an Appropriate Tool for Testing Experiments?", Proceedings of the $27^{\text {th }}$ International Conference on Software Engineering (ICSE'05), 2005.

[31]J. M. Spivey. Understanding Z: a specification language and its formal semantics. Cambridge University Press, 1988.

[32] J. R. Burch, E. M. Clarke, D. E. Long, "Representing circuits more efficiently in symbolic model checking". In proceedings of the $28^{\text {th }}$ IEEE Design Automation Conference, pp 403-407, 1991.

[33] J. R. Burch, E. M. Clarke, K. L. McMillan, D. L. Dill, "Sequential circuit verification using symbolic model checking". In proceedings of the $27^{\text {th }}$ IEEE Design Automation Conference, pp 46-51, 1990.

[34] K. L. McMillan, "A methodology for hardware verification using compositional model checking”, Science of Computer Programming, Vol.37, pp. 279-309, 2000.

[35]L. R. Ford, D. R. Fulkerson, Flows in Networks. Princeton University Press, 1962.

[36]Leslie Lamport, "The Temporal Logic of Actions". ACM Transactions on Programming Languages and Systems, pp. 872-923, Vol. 16, No. 3, May 1994. 
[37]Lionel Brand, Dietmar Pfahl, "Using Simulation for Assessing the Real Impact of Test Coverage on Defect Coverage". In proceedings of $10^{\text {th }}$ International Conference on Software Reliability Engineering (ISSRE), Boca Raton, Florida, 1999.

[38] M. C. Browne, E. M. Clarke, D. L. Dill, B. Mishra, "Automatic verification of sequential circuits using temporal logic". IEEE Transactions on Computers, pp 1035-1044, C-35, 12, 1986.

[39]M. W. Rohrer, "Seeing is Believing: The Importance of Visualization in Manufacturing Simulation". In proceedings of the 2000 Winter Simulation Conference, Orlando, Fl, 2000.

[40] M. Weiser, "Program Slicing". IEEE Transactions on Software Engineering, Vol. SE-10, No. 4, 1984, pp. 352-357.

[41] Martin Abadi, Leslie Lamport, "Conjoining Specifications". ACM Transactions on Programming Languages and Systems, pp. 507-534, Vol. 17, No. 3, May 1995.

[42] Mats P.E. Heimdahl, Devaraj George, Robert Weber, "Specification Test Coverage Adequacy Criteria = Specification Test Generation Inadequacy Criteria?". In proceedings of $8^{\text {th }}$ IEEE International Symposium on High Assurance Systems (HASE'04), Tampa, Florida, 2004.

[43] Mei-Hwa Chen, Michael R. Lyu, W. Eric Wong, "An Empirical Study of the Correlation between Code Coverage and Reliability Estimation". In proceedings of $3^{\text {rd }}$ International Software Metrics Symposium, pp 133-141, Berlin, Germany, 1996.

[44] Mei-Hwa Chen, Michael R. Lyu, W. Eric Wong, "Effect of Code Coverage on Software Reliability Measurment". IEEE Transactions on Reliability, pp 165 - 170, Vol. 50, No. 2, June 2001.

[45] Mike Benjamin, Daniel Geist, Alan Hartman, Yaron Wolfsthal, Gerard Mas, Ralph Smeets, "A Study in Coverage-Driven Test Generation". In proceedings of the 36th ACM/IEEE conference on Design automation, New Orleans, Louisiana, 1999.

[46] Myla Archer, Constance Heitmeyer, Elvinia Riccobene, "Proving Invariants of I/O Automata with TAME”. Automated Software Engineering, Vol. 9, 2002.

[47] N. Boudriga, F. Elloumi, A. Mili, "On the lattice of specifications: Applications to a specification methodology". Formal Aspects of Computing, vol. 4, pp. 544-571 1992.

[48] N. G. Leveson, "Software Safety: Why, What, and How". Computing Surveys, Vol. 18, No. 2, June 1986.

[49] Natasha Sharygina, Doron Peled, "A Combined Testing and Verification Approach for Software Reliability". In proceedings of the International Symposium on Formal Methods Europe (FME 2001), Berlin, Germany, March 12-16, 2001. 
[50]P. J. Courtois, D. L. Parnas, "Documentation for Safety Critical Software". In proceedings of 15th International Conference on Software Engineering, Baltimore, MD, 17- 21 May, 1993.

[51] Petar Popic, Dejan Desovski, Walid Abdelmoez, Bojan Cukic, "Error Propagation in the Reliability Analysis of Component Based Systems", Proceedings of the International Symposium of Software Reliability (ISSRE'05), 2005.

[52] Philip J. Boland, Harhsinder Singh, Bojan Cukic, "Comparing Partition and Random Testing via Majorization and Schur Functions". IEEE Transactions on Software Engineering, pp 88-94, Vol. 29, No. 1, 2003.

[53] Praerit Garg, "Investigating Coverage-Reliability Relationship and Sensitivity of Reliability to Errors in the Operational Profile". In proceedings of the IBM Centre for Advanced Studies on Collaborative research conference, IBM Press, Toronto, Ontario, Canada, 1994.

[54]R. Bharadwaj, S. Sims, "Salsa: Combining Constraint Solvers with BDDs for Automatic Invariant Checking", Proceedings of Tools and Algorithms for the Construction and Analysis of Systems, 2000.

[55]R. E. Bryant, "Graph-based algorithms for Boolean function manipulation”. IEEE Transactions on Computers, pp 677-691, C-35, 8, 1986.

[56] R. Hamlet. Random Testing. Encyclopedia of Software Engineering, Wiley, 1994.

[57]R. Motwani, P. Raghavan. Randomized Algorithms. Cambridge University Press, 1995.

[58] Rajeev Alur, Robert K. Brayton, Thomas A. Henzinger, Shaz Qadeer, and Sriram K. Rajamani, "Partial-Order Reduction in Symbolic State-Space Exploration". Formal Methods in System Design 18:97-116, 2001.

[59] Ralph D. Jeffords, Constance L. Heitmeyer, "A Strategy for Efficiently Verifying Requirements Specifications Using Composition and Invariants". In proceedings of $9^{\text {th }}$ European Software Engineering Conference held jointly with $11^{\text {th }}$ International Symposium on Foundations of Software Engineering (ESEC/FSE'03), Helsinki, Finland, 1-5 September, 2003.

[60] Ralph Jeffords, Constance Heitmeyer, "Automatic Generation of State Invariants from Requirements Specifications". In proceedings of $6^{\text {th }}$ International Symposium on Foundations of Software Engineering (FSE'98), Orlando, FL, 3-5 November, 1998.

[61]Ramesh Bharadwaj, Constance L. Heitmeyer, "Model Checking Complete Requirements Specifications Using Abstraction". Automated Software Engineering, Vol. 6, 1999, pp. 37-68. 
[62] Richard Hamlet, "Theoretical Comparison of Testing Methods". In proceedings of ACM SIGSOFT International Symposium on Software Testing, Analysis and Verification, Key West, Florida, 1989.

[63] S. Antoy, R. Hamlet, "Automatically Checking an Implementation against Its Formal Specification”, IEEE Transactions on Software Engineering, vol. 26, no. 1, 2000.

[64] S. Skiena, Implementing Discrete Mathematics: Combinatorics and Graph Theory with Mathematica. Addison-Wesley, 1990.

[65] Saileshwar Krishnamurthy, Aditya P. Mathur, "On Predicting Reliability of Modules Using Code Coverage". In proceedings of the IBM Centre for Advanced Studies on Collaborative research conference, IBM Press, Toronto, Ontario, Canada, 1996.

[66] Simeon C. Ntafos, "A Comparison of Some Structural testing Strategies". IEEE Transactions on Software Engineering, Vol 14, No 6, pp 868-874, June 1988.

[67] Stacy J. Prowell, Jesse H. Poore, "Foundations of Sequence-Based Software Specification". IEEE Transactions on Software Engineering, Vol. 29, No. 5, May 2003.

[68] Thomas A. Henzinger, Shaz Qadeer, Sriram K. Rajamani, "Decomposing Refinement Proofs using Assume-Guarantee Reasoning". In proceedings of the IEEE/ACM International Conference on Computer-aided Design (ICCAD'00), pp. 245-252, 2000.

[69] Thomas A. Henzinger, Shaz Qadeer, Sriram K. Rajamani, "You Assume, We Guarantee: Methodology and Case Studies". In proceedings of the $10^{\text {th }}$ International Conference on Computer-Aided Verification (CAV), pp. 440-451, 1998.

[70] Victor R. Basili, Richard W. Selby, "Comparing the Effectiveness of Software Testing Strategies". IEEE Transactions on Software Engineering, pp 1278-1296, Vol. SE-13, No. 12, December 1987.

[71] Victor Winter, Dejan Desovski, Bojan Cukic, "Virtual Environment Modeling for Requirements Validation of High Consequence Systems". In proceedings of $5^{\text {th }}$ IEEE International Symposium on Requirements Engineering (RE 2001), 27-31 August 2001, Toronto, Canada, pp. 23-30.

[72] Yashwant K. Malaiya, Michael Naixin Li, James M. Bieman, Rick Karcich, "Software Reliability Growth with Test Coverage". IEEE Transactions on Reliability, pp 420 - 426, Vol. 51, No. 4, December 2002.

[73]Z. Manna, A. Pnueli. The Temporal Logic of Reactive and Concurrent Systems: Specification. Springer-Verlag, 1992. 
[74] Requirements Specification for Personnel Access Control System. National Security Agency, 2003.

[75] http://pvs.csl.sri.com/

[76] http://theory.lcs.mit.edu/ mslevine/mincut/

[77] http://www.altia.com/

[78] http://www.ilog.com/products/jloox/

[79] http://www.ilog.com/products/jviews/

[80] http://www.bullseye.com/coverage.html 


\section{Appendix}

\section{A.1. Original PACS SCR specification}

\begin{tabular}{|l|l|l|l|l|}
\hline \multicolumn{2}{|c|}{ Type Dictionary } & Comment \\
\hline Name & Base Type & Units & Legal Values & \\
\hline yAlarm & Enumerated & On, Off & \\
\hline yGate & Enumerated & Open, Closed & \\
\hline yGuardDisplay & Enumerated & Blank, SeeOfficer & \\
\hline yKeys & Enumerated & Blank, Number, Delete & \\
\hline yUserDisplay & Enumerated & & $\begin{array}{l}\text { InsertCard, Retry, EnterPIN, InvalidPIN, PleaseProceed, } \\
\text { SeeOfficer }\end{array}$ & \\
\hline
\end{tabular}

\section{Mode Class Dictionary}

\begin{tabular}{|l|l|l|l|}
\hline Name & Modes & $\begin{array}{l}\text { Initial } \\
\text { Mode }\end{array}$ & Comment \\
\hline mcPIN & Init, GetDigit1, GetDigit2, GetDigit3, GetDigit4 & Init & \\
\hline mcStatus & $\begin{array}{l}\text { EnterCard, CheckCard, EnterPIN, CheckPIN, Proceed, ReEnterCard, } \\
\text { ReEnterPIN, Error, Override }\end{array}$ & EnterCard & \\
\hline
\end{tabular}

\begin{tabular}{|l|l|l|l|}
\hline \multicolumn{4}{c|}{ Constant Dictionary } \\
\hline Name & Type & Value & Comment \\
\hline MaxCardError & Integer & 3 & \\
\hline MaxPINError & Integer & 3 & \\
\hline
\end{tabular}

\begin{tabular}{|l|l|l|l|l|}
\hline \multicolumn{9}{|c|}{ Monitored Variable Dictionary } \\
\hline Name & Type & Initial Value & Accuracy & Comment \\
\hline mCardInput & Boolean & FALSE & & \\
\hline mCardValid & Boolean & FALSE & & \\
\hline mDigit1 & yKeys & Blank & & \\
\hline mDigit2 & yKeys & Blank & & \\
\hline mDigit3 & yKeys & Blank & & \\
\hline mDigit4 & yKeys & Blank & & \\
\hline mGate & yGate & Closed & & \\
\hline mOverride & Boolean & FALSE & & \\
\hline mPINValid & Boolean & FALSE & & \\
\hline mReset & Boolean & FALSE & & \\
\hline
\end{tabular}

\section{Term Dictionary}

\begin{tabular}{|l|l|l|l|l|}
\hline Name & Type & Initial Value & Accuracy & Comment \\
\hline mPINInput & Boolean & FALSE & & \\
\hline tNumCReads & Integer & 0 & & \\
\hline tNumPReads & Integer & 0 & & \\
\hline
\end{tabular}




\begin{tabular}{|l|l|l|l|l|}
\hline \multicolumn{5}{|c|}{ Controlled Variable Dictionary } \\
\hline Name & Type & Initial Value & Accuracy & Comment \\
\hline cGate & yGate & Closed & & \\
\hline cGuardAlarm & yAlarm & Off & & \\
\hline cGuardDisplay & yGuardDisplay & Blank & & \\
\hline cUserDisplay & yUserDisplay & InsertCard & & \\
\hline
\end{tabular}

Assertion Dictionary

\begin{tabular}{|c|c|c|c|c|}
\hline Name & Source & $\begin{array}{l}\text { Proof } \\
\text { Result }\end{array}$ & Expression & Comment \\
\hline AlarmStatus & User & - & $($ cGuardAlarm $=$ On $) \Leftrightarrow>$ (cUserDisplay = SeeOfficer $)$ & \\
\hline CardDisplay1 & User & - & $\begin{array}{l}\text { (tNumCReads }>0 \text { AND tNumCReads }<\text { MaxCardError })<=> \\
\text { (cUserDisplay }=\text { Retry) }\end{array}$ & \\
\hline CardDisplay2 & User & - & $\begin{array}{l}\text { (tNumCReads = MaxCardError) }=>\text { (cUserDisplay }= \\
\text { SeeOfficer })\end{array}$ & \\
\hline CardErrors & User & - & $\begin{array}{l}((\text { cUserDisplay }=\text { InsertCard OR cUserDisplay }=\text { Retry }) \\
\text { AND mcStatus }=\text { CheckCard AND @F }(\text { mCardValid }))=> \\
(\text { tNumCReads' }=\text { tNumCReads }+1)\end{array}$ & \\
\hline CardSuccess & User & - & $\begin{array}{l}((\text { cUserDisplay }=\text { InsertCard OR cUserDisplay }=\text { Retry }) \\
\text { AND mcStatus }=\text { CheckCard AND @ }(\text { mCardValid }))=> \\
(\text { cUserDisplay' }=\text { EnterPIN })\end{array}$ & \\
\hline GateStatus & User & - & (cUserDisplay = PleaseProceed) $<=>($ cGate $=$ Open $)$ & \\
\hline NumCardErrors & User & - & (tNumCReads <= MaxCardError) & \\
\hline NumPINErrors & User & - & (tNumPReads <= MaxPINError) & \\
\hline PINDisplay1 & User & - & $\begin{array}{l}\text { (tNumPReads }>0 \text { AND tNumPReads }<\text { MaxPINError })<=> \\
\text { (cUserDisplay }=\text { InvalidPIN) }\end{array}$ & \\
\hline PINDisplay2 & User & - & $\begin{array}{l}\text { (tNumPReads }=\text { MaxPINError })=>(\mathrm{cUserDisplay}= \\
\text { SeeOfficer })\end{array}$ & \\
\hline PINEntry & User & - & $\begin{array}{l}(@ \mathrm{C}(\mathrm{mPINInput})=>(\mathrm{mcStatus}=\text { EnterPIN OR mcStatus }= \\
\text { ReEnterPIN }))\end{array}$ & \\
\hline PINErrors & User & 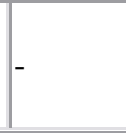 & $\begin{array}{l}((\text { cUserDisplay }=\text { EnterPIN OR cUserDisplay }=\text { InvalidPIN }) \\
\text { AND mcStatus }=\text { CheckPIN AND @ }(\text { FPINValid }))=> \\
(\text { tNumPReads' }=\text { tNumPReads }+1)\end{array}$ & \\
\hline PINSuccess & User & - & $\begin{array}{l}((\text { cUserDisplay }=\text { EnterPIN OR cUserDisplay }=\text { InvalidPIN }) \\
\text { AND mcStatus }=\text { CheckPIN AND @ }(\text { mPINValid }))=> \\
(\text { cUserDisplay' }=\text { PleaseProceed })\end{array}$ & \\
\hline Reset & User & - & $((@ \mathrm{C}(\mathrm{mReset}))=>$ (cUserDisplay' = InsertCard $)$ & \\
\hline Safety & User & - & $\begin{array}{l}\text { (cUserDisplay }=\text { SeeOfficer })<=>\text { (cGuardDisplay }= \\
\text { SeeOfficer })\end{array}$ & \\
\hline
\end{tabular}

\section{Assumption Dictionary}

\begin{tabular}{|l|l|l|}
\hline Name & Expression & Comment \\
\hline GateOpen & $@ \mathrm{~T}(\mathrm{mGate}=$ Open $)=>($ cGate $=$ Open $)$ & \\
\hline PINInput & $@ \mathrm{C}(\mathrm{mPINInput})=>(\mathrm{mcPIN}=$ GetDigit4 AND @ $(\mathrm{mDigit} 4=$ Number $))$ & \\
\hline
\end{tabular}




\begin{tabular}{|l|l|c|}
\hline \multicolumn{2}{|c|}{ cGate Condition Function } \\
\hline & \multicolumn{1}{|c|}{ Conditions } \\
\hline & $\begin{array}{l}\text { mcStatus = Proceed OR mcStatus }= \\
\text { Override }\end{array}$ & $\begin{array}{l}\text { NOT (mcStatus = Proceed OR mcStatus = } \\
\text { Override })\end{array}$ \\
\hline cGate $=$ & Open & Closed \\
\hline
\end{tabular}

\begin{tabular}{|l|l|l|}
\hline \multicolumn{2}{|c|}{ cGuardAlarm Condition Function } \\
\hline & \multicolumn{1}{|c|}{ Conditions } \\
\hline & mcStatus = Error & NOT (mcStatus = Error $)$ \\
\hline cGuardAlarm $=$ & On & Off \\
\hline
\end{tabular}

\begin{tabular}{|l|l|l|}
\hline \multicolumn{3}{|c|}{ cGuardDisplay Event Function } \\
\hline & \multicolumn{1}{|c|}{ Events } \\
\hline & $@ \mathrm{~T}(\mathrm{mcStatus}=$ EnterCard $)$ OR @ $\mathrm{T}(\mathrm{mcStatus}=$ Override $)$ & $@ \mathrm{~T}(\mathrm{mcStatus}=$ Error $)$ \\
\hline cGuardDisplay' $=$ & Blank & SeeOfficer \\
\hline
\end{tabular}

\begin{tabular}{|c|c|c|c|c|c|c|}
\hline \multicolumn{7}{|c|}{ cUserDisplay Event Function } \\
\hline & \multicolumn{6}{|c|}{$\begin{array}{ll}\text { Events } \\
\end{array}$} \\
\hline & $\begin{array}{l}@ \mathrm{~T}(\mathrm{mcStatus} \\
=\text { EnterCard })\end{array}$ & $\begin{array}{l}@ \mathrm{~T}(\mathrm{mcStatus} \\
= \\
\text { ReEnterCard })\end{array}$ & $\begin{array}{l}@ \mathrm{~T}(\mathrm{mcStatus} \\
=\text { EnterPIN })\end{array}$ & $\begin{array}{l}@ \mathrm{~T}(\mathrm{mcStatus} \\
= \\
\text { ReEnterPIN })\end{array}$ & $\begin{array}{l}@ \mathrm{~T}(\mathrm{mcStatus} \\
=\text { Proceed }) \\
\text { OR } \\
@ \mathrm{~T}(\mathrm{mcStatus} \\
=\text { Override })\end{array}$ & $\begin{array}{l}@ \mathrm{~T}(\mathrm{mcStatus} \\
=\text { Error })\end{array}$ \\
\hline cUserDisplay' = & InsertCard & Retry & EnterPIN & InvalidPIN & PleaseProceed & SeeOfficer \\
\hline
\end{tabular}

\begin{tabular}{|c|c|c|}
\hline \multicolumn{3}{|c|}{ mcPIN Mode Transition Function } \\
\hline Source Mode(s) & Events & Destination Mode \\
\hline Init & $\begin{array}{l}\text { @ } \mathrm{T}(\mathrm{m} \text { CardValid) WHEN (mcStatus }=\text { CheckCard) } \\
\text { OR @F(mPINValid) WHEN (mcStatus = CheckPIN } \\
\text { AND tNumPReads }<\text { MaxPINError }-1)\end{array}$ & GetDigit1 \\
\hline GetDigit1 & $@ \mathrm{~T}(\mathrm{mDigit} 1$ = Number $)$ & GetDigit2 \\
\hline GetDigit2 & $@ \mathrm{~T}(\mathrm{mDigit} 2=$ Number $)$ & GetDigit3 \\
\hline GetDigit2 & $@ \mathrm{~T}(\mathrm{mDigit} 2=$ Delete $)$ & GetDigit1 \\
\hline GetDigit3 & $@ \mathrm{~T}(\mathrm{mDigit} 3=$ Number $)$ & GetDigit4 \\
\hline GetDigit3 & $@ \mathrm{~T}(\mathrm{mDigit} 3=$ Delete $)$ & GetDigit2 \\
\hline GetDigit4 & $@ \mathrm{~T}(\mathrm{mDigit} 4=$ Number $)$ & Init \\
\hline GetDigit4 & $@ \mathrm{~T}(\mathrm{mDigit} 4=$ Delete $)$ & GetDigit3 \\
\hline $\begin{array}{l}\text { GetDigit1, GetDigit2, } \\
\text { GetDigit3, GetDigit4 }\end{array}$ & $@ \mathrm{C}(\mathrm{mReset})$ & Init \\
\hline
\end{tabular}




\begin{tabular}{|c|c|c|}
\hline \multicolumn{3}{|c|}{ mcStatus Mode Transition Function } \\
\hline Source Mode(s) & Events & Destination Mode \\
\hline EnterCard & $@ \mathrm{C}(\mathrm{mCardInput})$ & CheckCard \\
\hline CheckCard & $@ \mathrm{~T}(\mathrm{mCardValid})$ & EnterPIN \\
\hline EnterPIN & @C(mPINInput) & CheckPIN \\
\hline CheckPIN & @T(mPINValid) & Proceed \\
\hline Proceed & $@ \mathrm{~T}(\mathrm{mGate}=\mathrm{Closed})$ & EnterCard \\
\hline CheckCard & $\begin{array}{l}@ \mathrm{~F}(\mathrm{mCardValid}) \mathrm{WHEN} \text { (tNumCReads < } \\
\text { MaxCardError - 1) }\end{array}$ & ReEnterCard \\
\hline ReEnterCard & $@ \mathrm{C}(\mathrm{mCardInput)}$ & CheckCard \\
\hline CheckCard & $\begin{array}{l}@ \mathrm{~F}(\mathrm{mCardValid}) \mathrm{WHEN}(\mathrm{tNumCReads}= \\
\text { MaxCardError - } 1)\end{array}$ & Error \\
\hline CheckPIN & $\begin{array}{l}@ \mathrm{~F}(\mathrm{mPINV} \text { alid) WHEN (tNumPReads < } \\
\text { MaxPINError - 1) }\end{array}$ & ReEnterPIN \\
\hline ReEnterPIN & $@ \mathrm{C}(\mathrm{mPINInput)}$ & CheckPIN \\
\hline CheckPIN & $\begin{array}{l}@ \mathrm{~F}(\mathrm{mPINV} \text { alid) WHEN (tNumPReads = } \\
\text { MaxPINError - 1) }\end{array}$ & Error \\
\hline Error & $@ \mathrm{C}(\mathrm{mOverride})$ & Override \\
\hline Override & $@ \mathrm{~T}(\mathrm{mGate}=\mathrm{Closed})$ & EnterCard \\
\hline $\begin{array}{l}\text { EnterCard, CheckCard, } \\
\text { EnterPIN, CheckPIN, } \\
\text { ReEnterCard, } \\
\text { ReEnterPIN, Error, } \\
\text { Override, Proceed }\end{array}$ & $@ \mathrm{C}(\mathrm{mReset})$ & EnterCard \\
\hline
\end{tabular}

\begin{tabular}{|l|l|}
\hline \multicolumn{1}{|c|}{ mPINInput Event Function } \\
\hline & \multicolumn{1}{|c|}{ Events } \\
\hline & @T(mDigit4 = Number) WHEN (mcPIN = GetDigit4) \\
\hline mPINInput' $=$ & NOT mPINInput \\
\hline
\end{tabular}

\begin{tabular}{|l|l|l|}
\hline \multicolumn{2}{|c|}{ tNumCReads Event Function } \\
\hline $\begin{array}{l}\text { Modes for } \\
\text { mcStatus }\end{array}$ & \multicolumn{2}{|c|}{ Events } \\
\hline CheckCard & $@ \mathrm{~T}(\mathrm{mCardValid}) \mathrm{OR} @ \mathrm{C}(\mathrm{mReset})$ & @F(mCardValid) \\
\hline Error & $@ \mathrm{C}(\mathrm{mOv}$ (mride) OR @C(mReset) & NEVER \\
\hline ReEnterCard & $@ \mathrm{C}(\mathrm{mReset})$ & NEVER \\
\hline tNumCReads' $=$ & 0 & tNumCReads +1 \\
\hline
\end{tabular}

\begin{tabular}{|l|l|l|}
\hline \multicolumn{3}{|c|}{ tNumPReads Event Function } \\
\hline $\begin{array}{l}\text { Modes for } \\
\text { mcStatus }\end{array}$ & \multicolumn{1}{|c|}{ Events } \\
\hline CheckPIN & @T(mPINValid) OR @C(mReset $)$ & @F(mPINValid) \\
\hline Error & $@ \mathrm{C}(\mathrm{mOverride}) \mathrm{OR} @ \mathrm{C}(\mathrm{mReset})$ & NEVER \\
\hline ReEnterPIN & @C(mReset $)$ & NEVER \\
\hline tNumPReads' $=$ & 0 & tNumPReads +1 \\
\hline
\end{tabular}




\section{A.2. Redesigned PACS SCR specification}

\section{Type Dictionary}

\begin{tabular}{|l|l|l|l|l|}
\hline Name & Base Type & Units & Legal Values & Comment \\
\hline yAlarm & Enumerated & On, Off & \\
\hline yGate & Enumerated & Open, Closed & \\
\hline yGuardDisplay & Enumerated & & Blank, SeeOfficer & \\
\hline yKeys & Enumerated & & Blank, Number, Delete & \\
\hline yUserDisplay & Enumerated & & $\begin{array}{l}\text { InsertCard, Retry, EnterPIN, InvalidPIN, PleaseProceed, } \\
\text { SeeOfficer }\end{array}$ & \\
\hline yValid & Enumerated & & Unknown, Yes, No, Error & \\
\hline
\end{tabular}

\section{Mode Class Dictionary}

\begin{tabular}{|l|l|l|l|}
\hline Name & Modes & Initial Mode & Comment \\
\hline mcCard & WaitSwap, ReadData, Error & WaitSwap & \\
\hline mcPIN & GetDigit1, GetDigit2, GetDigit3, GetDigit4, CheckPIN, Error & GetDigit1 & \\
\hline mcStatus & CheckCard, CheckPIN, Proceed, Error & CheckCard & \\
\hline
\end{tabular}

\section{Constant Dictionary}

\begin{tabular}{|l|l|l|l|}
\hline Name & Type & Value & Comment \\
\hline MaxCardError & Integer & 3 & \\
\hline MaxPINError & Integer & 3 & \\
\hline
\end{tabular}

\section{Monitored Variable Dictionary}

\begin{tabular}{|l|l|l|l|l|}
\hline Name & Type & Initial Value & Accuracy & Comment \\
\hline mCardInput & Boolean & FALSE & & \\
\hline mCardValid & Boolean & FALSE & & \\
\hline mDigit1 & yKeys & Blank & & \\
\hline mDigit2 & yKeys & Blank & & \\
\hline mDigit3 & yKeys & Blank & & \\
\hline mDigit4 & yKeys & Blank & & \\
\hline mGate & yGate & Closed & & \\
\hline mPINValid & Boolean & FALSE & & \\
\hline
\end{tabular}

Term Dictionary

\begin{tabular}{|l|l|l|l|l|}
\hline Name & Type & Initial Value & Accuracy & Comment \\
\hline tCardValid & yValid & Unknown & & \\
\hline tNumCReads & Integer & 0 & & \\
\hline tNumPReads & Integer & 0 & & \\
\hline tPINValid & yValid & Unknown & & \\
\hline
\end{tabular}




\begin{tabular}{|l|l|l|l|l|}
\hline \multicolumn{4}{|c|}{ Controlled Variable Dictionary } \\
\hline Name & Type & Initial Value & Accuracy & Comment \\
\hline cGate & yGate & Closed & & \\
\hline cGuardAlarm & yAlarm & Off & & \\
\hline cGuardDisplay & yGuardDisplay & Blank & & \\
\hline cUserDisplay & yUserDisplay & InsertCard & & \\
\hline
\end{tabular}

\section{Assertion Dictionary}

\begin{tabular}{|c|c|c|c|c|}
\hline Name & Source & $\begin{array}{l}\text { Proof } \\
\text { Result }\end{array}$ & Expression & Comment \\
\hline AlarmStatus & User & - & $($ cGuardAlarm = On $)<=>$ (cUserDisplay = SeeOfficer $)$ & \\
\hline CardDisplay1 & User & - & $\begin{array}{l}\text { (tNumCReads }>0 \text { AND tNumCReads }<\text { MaxCardError })<= \\
\text { (cUserDisplay }=\text { Retry) }\end{array}$ & \\
\hline CardDisplay2 & User & - & $\begin{array}{l}\text { (tNumCReads }=\text { MaxCardError })=>(\text { cUserDisplay }= \\
\text { SeeOfficer })\end{array}$ & \\
\hline CardErrors & User & - & $\begin{array}{l}((\text { cUserDisplay }=\text { InsertCard OR cUserDisplay }=\text { Retry }) \\
\text { AND mcStatus }=\text { CheckCard AND @ }(\text { tCardValid = No })) \\
=>(\text { tNumCReads' }=\text { tNumCReads }+1)\end{array}$ & \\
\hline CardSuccess & User & - & $\begin{array}{l}((\text { cUserDisplay = InsertCard OR cUserDisplay = Retry }) \\
\text { AND mcStatus = CheckCard AND @ }(\text { tCardValid = Yes })) \\
=>(\text { cUserDisplay' = EnterPIN })\end{array}$ & \\
\hline GateStatus & User & - & $($ cUserDisplay $=$ PleaseProceed $)<=>($ cGate $=$ Open $)$ & \\
\hline NumCardErrors & User & - & (tNumCReads <= MaxCardError) & \\
\hline NumPINErrors & User & - & (tNumPReads <= MaxPINError) & \\
\hline PINDisplay1 & User & - & $\begin{array}{l}\text { (tNumPReads }>0 \text { AND tNumPReads }<\text { MaxPINError })<=> \\
(\text { cUserDisplay }=\text { InvalidPIN })\end{array}$ & \\
\hline PINDisplay2 & User & - & $\begin{array}{l}\text { (tNumPReads }=\text { MaxPINError })=>(\text { cUserDisplay }= \\
\text { SeeOfficer })\end{array}$ & \\
\hline PINEntry & User & - & $((@ \mathrm{C}(\mathrm{tPINValid})=>($ mcStatus $=$ CheckPIN $))$ & \\
\hline PINErrors & User & - & 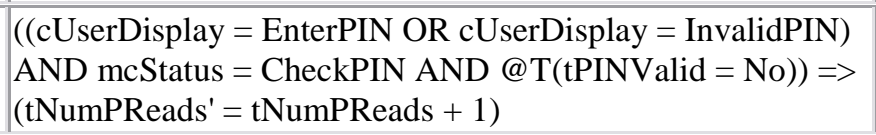 & \\
\hline PINSuccess & User & - & $\begin{array}{l}((\text { UUserDisplay }=\text { EnterPIN OR cUserDisplay }=\text { InvalidPIN }) \\
\text { AND mcStatus }=\text { CheckPIN AND @T }(\text { tPINValid }=\text { Yes })) \Rightarrow \\
(\text { cUserDisplay' }=\text { PleaseProceed })\end{array}$ & \\
\hline Safety & User & - & $\begin{array}{l}\text { (cUserDisplay }=\text { SeeOfficer }) \Leftrightarrow=>(\text { cGuardDisplay }= \\
\text { SeeOfficer })\end{array}$ & \\
\hline
\end{tabular}

\section{Assumption Dictionary}

\begin{tabular}{|l|l|l|}
\hline Name & Expression & Comment \\
\hline GateOpen & $@ T(m G a t e=$ Open $)=>($ cGate $=$ Open $)$ & \\
\hline
\end{tabular}

\begin{tabular}{|l|l|l|}
\hline \multicolumn{2}{|c|}{ cGate Condition } & \multicolumn{1}{|c|}{ Function } \\
\hline & \multicolumn{1}{|c|}{ Conditions } \\
\hline & mcStatus = Proceed & NOT $($ mcStatus = Proceed $)$ \\
\hline cGate $=$ & Open & Closed \\
\hline
\end{tabular}




\begin{tabular}{|l|l|l|}
\hline \multicolumn{2}{|c|}{ cGuardAlarm Condition Function } \\
\hline & \multicolumn{2}{|c|}{ Conditions } \\
\hline & mcStatus = Error & NOT (mcStatus = Error) \\
\hline cGuardAlarm $=$ & On & Off \\
\hline
\end{tabular}

\begin{tabular}{|l|l|l|}
\hline \multicolumn{2}{|c|}{ cGuardDisplay Event Function } \\
\hline & \multicolumn{1}{|c|}{ Events } \\
\hline & @T(mcStatus = CheckCard $)$ & @ T(mcStatus = Error $)$ \\
\hline cGuardDisplay' $=$ & Blank & SeeOfficer \\
\hline
\end{tabular}

\begin{tabular}{|c|c|c|c|c|c|c|}
\hline \multicolumn{7}{|c|}{ cUserDisplay Event Function } \\
\hline & \multicolumn{6}{|c|}{ Events } \\
\hline & $\begin{array}{l}@ \mathrm{~T}(\mathrm{mcStatus} \\
= \\
\text { CheckCard) }\end{array}$ & $\begin{array}{l}\text { @T(tCardValid } \\
=\text { No) WHEN } \\
\text { (mcStatus = } \\
\text { CheckCard) }\end{array}$ & $\begin{array}{l}@ \mathrm{~T}(\mathrm{mcStatus} \\
=\mathrm{CheckPIN})\end{array}$ & $\begin{array}{l}@ \mathrm{~T}(\mathrm{tPINV} \text { alid } \\
=\mathrm{No}) \mathrm{WHEN} \\
\text { (mcStatus = } \\
\text { CheckPIN) }\end{array}$ & $\begin{array}{l}@ \mathrm{~T}(\mathrm{mcStatus} \\
=\text { Proceed })\end{array}$ & $\begin{array}{l}@ \mathrm{~T}(\mathrm{mcStatus} \\
=\text { Error })\end{array}$ \\
\hline cUserDisplay' = & InsertCard & Retry & EnterPIN & InvalidPIN & PleaseProceed & SeeOfficer \\
\hline
\end{tabular}

\begin{tabular}{|l|l|l|}
\hline \multicolumn{3}{|c|}{ mcCard Mode Transition Function } \\
\hline Source Mode(s) & Events & Destination Mode \\
\hline WaitSwap & $@ \mathrm{~T}(\mathrm{mCardInput})$ WHEN (mcStatus = CheckCard) & ReadData \\
\hline ReadData & $\begin{array}{l}\text { @T(mCardValid) OR @ F(mCardValid) WHEN } \\
\text { (tNumCReads < MaxCardError - 1) }\end{array}$ & WaitSwap \\
\hline ReadData & $@ \mathrm{~T}($ tNumCReads = MaxCardError $)$ & Error \\
\hline
\end{tabular}

\begin{tabular}{|c|c|c|}
\hline \multicolumn{3}{|c|}{ mcPIN Mode Transition Function } \\
\hline Source Mode(s) & Events & Destination Mode \\
\hline GetDigit1 & $\begin{array}{l}@ \mathrm{~T}(\mathrm{mDigit} 1 \text { = Number }) \text { WHEN }(\mathrm{mcStatus}= \\
\text { CheckPIN })\end{array}$ & GetDigit2 \\
\hline GetDigit2 & $@ \mathrm{~T}(\mathrm{mDigit} 2=$ Number $)$ & GetDigit3 \\
\hline GetDigit2 & $@ \mathrm{~T}(\mathrm{mDigit} 2=$ Delete $)$ & GetDigit1 \\
\hline GetDigit3 & $@ \mathrm{~T}(\mathrm{mDigit} 3=$ Number $)$ & GetDigit4 \\
\hline GetDigit3 & $@ \mathrm{~T}(\mathrm{mDigit} 3=$ Delete $)$ & GetDigit2 \\
\hline GetDigit4 & $@ \mathrm{~T}(\mathrm{mDigit} 4=$ = Number $)$ & CheckPIN \\
\hline GetDigit4 & $@ \mathrm{~T}(\mathrm{mDigit} 4=$ Delete $)$ & GetDigit3 \\
\hline CheckPIN & $\begin{array}{l}\text { @T(mPINValid) OR @F(mPINValid) WHEN } \\
\text { (tNumPReads < MaxPINError - 1) }\end{array}$ & GetDigit1 \\
\hline CheckPIN & $@ \mathrm{~T}(\mathrm{tNumPReads}=$ MaxPINError) & Error \\
\hline
\end{tabular}




\begin{tabular}{|l|l|l|}
\hline \multicolumn{2}{|c|}{ mcStatus Mode Transition Function } \\
\hline Source Mode(s) & Events & Destination Mode \\
\hline CheckCard & $@ \mathrm{~T}(\mathrm{tCardValid}=$ Yes $)$ & CheckPIN \\
\hline CheckCard & $@ \mathrm{~T}(\mathrm{tCardValid}=$ Error $)$ & Error \\
\hline CheckPIN & $@ \mathrm{~T}(\mathrm{tPINValid}=$ Yes $)$ & Proceed \\
\hline CheckPIN & $@ \mathrm{~T}(\mathrm{tPINValid}=$ Error $)$ & Error \\
\hline Proceed & @T(mGate = Closed $)$ & CheckCard \\
\hline
\end{tabular}

\begin{tabular}{|l|l|l|l|l|}
\hline \multicolumn{5}{|c|}{ tCardValid Event Function } \\
\hline & \multicolumn{3}{|c|}{ Events } \\
\hline & $\begin{array}{l}\text { @T(mCardValid) } \\
\text { WHEN (mcCard } \\
\text { ReadData })\end{array}$ & $\begin{array}{l}\text { @ F(mCardValid) WHEN } \\
\text { (mcCard = ReadData } \\
\text { AND tNumCReads }< \\
\text { MaxCardError }-1)\end{array}$ & $\begin{array}{l}\text { @ T(tNumCReads = } \\
\text { MaxCardError })\end{array}$ & $\begin{array}{l}\text { @ }(\mathrm{mcCard} \\
=\text { ReadData })\end{array}$ \\
\hline tCardValid' $=$ & Yes & No & Error & Unknown \\
\hline
\end{tabular}

\begin{tabular}{|l|l|l|}
\hline \multicolumn{2}{|c|}{ tNumCReads Event Function } \\
\hline Modes for mcCard & \multicolumn{2}{|c|}{ Events } \\
\hline ReadData & $@ \mathrm{~T}($ mCardValid) & @F(mCardValid) \\
\hline tNumCReads' $=$ & 0 & tNumCReads +1 \\
\hline
\end{tabular}

\begin{tabular}{|l|l|l|}
\hline \multicolumn{2}{|c|}{ tNumPReads Event Function } \\
\hline Modes for mcPIN & \multicolumn{1}{|c|}{ Events } \\
\hline CheckPIN & @ T(mPINValid) & @ F(mPINValid) \\
\hline tNumPReads' $=$ & 0 & tNumPReads + 1 \\
\hline
\end{tabular}

\begin{tabular}{|c|c|c|c|c|}
\hline \multicolumn{5}{|c|}{ tPINValid Event Function } \\
\hline & \multicolumn{4}{|c|}{ Events } \\
\hline & $\begin{array}{l}\text { @T(mPINValid) } \\
\text { WHEN (mcPIN = } \\
\text { CheckPIN) }\end{array}$ & $\begin{array}{l}\text { @ F(mPINValid) WHEN } \\
\text { (mcPIN = CheckPIN and } \\
\text { tNumPReads < } \\
\text { MaxPINError - 1) }\end{array}$ & $\begin{array}{l}\text { @ } \mathrm{T}(\mathrm{tNumPReads}= \\
\text { MaxPINError) }\end{array}$ & $\begin{array}{l}@ \mathrm{~T}(\mathrm{mcPIN}= \\
\text { CheckPIN })\end{array}$ \\
\hline tPINValid' = & Yes & No & Error & Unknown \\
\hline
\end{tabular}

